\title{
Functionalized Oxepines via Fragmentation of Highly Strained Epoxides
}

\author{
Andrew J. Leyhane and Marc L. Snapper \\ Department of Chemistry, Merkert Chemistry Center, Boston College, 2609 Beacon Street, \\ Chestnut Hill, MA 02467 \\ marc.snapper@BC.edu
}

Table of Contents

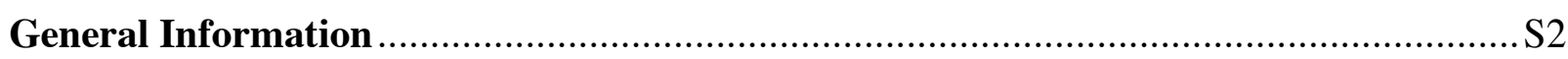

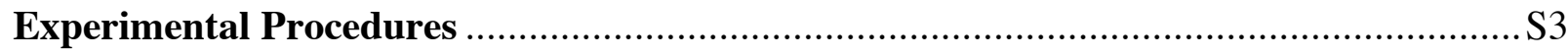

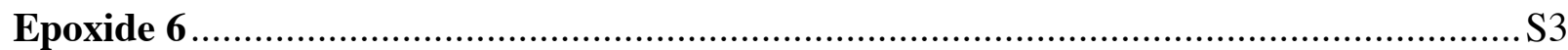

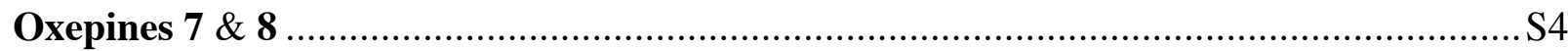

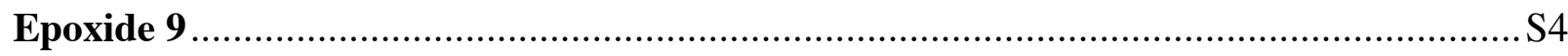

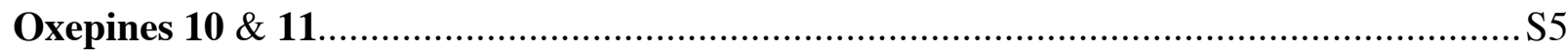

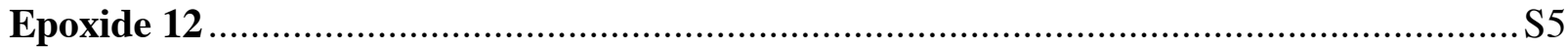

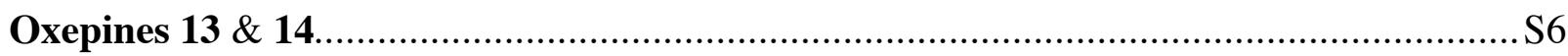

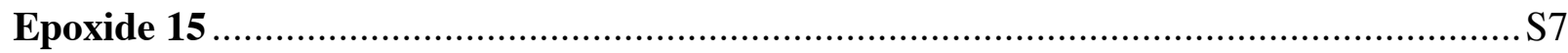

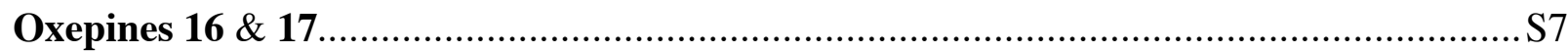

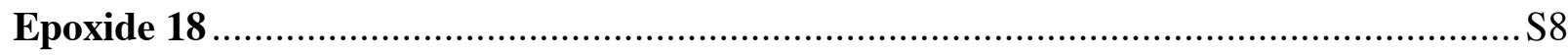

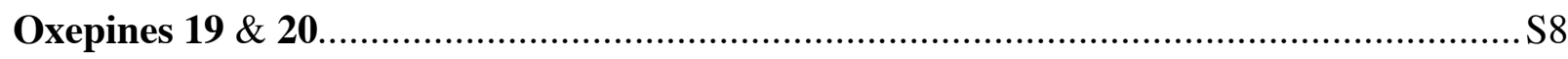

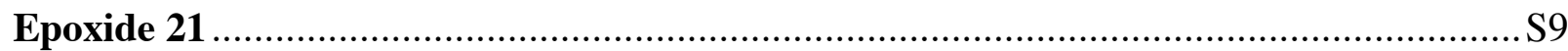

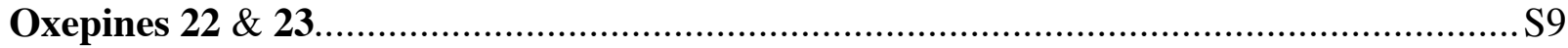

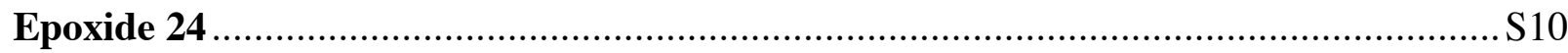

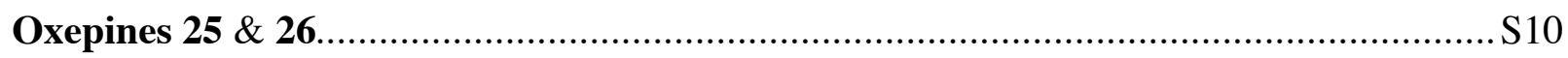

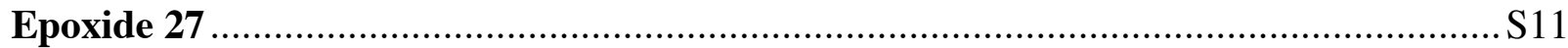

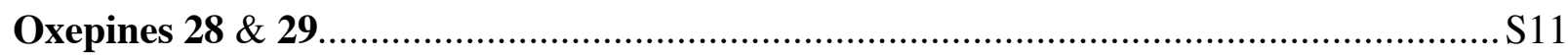

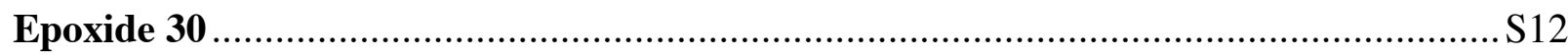

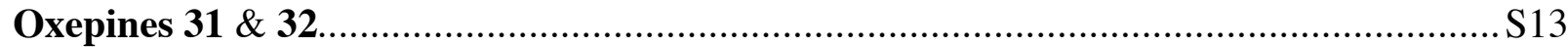

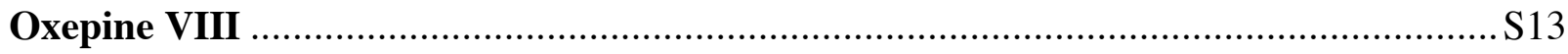

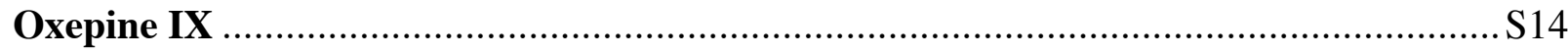

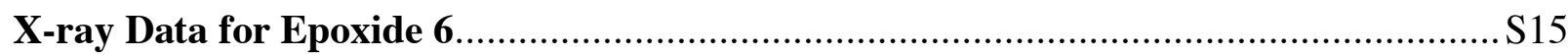

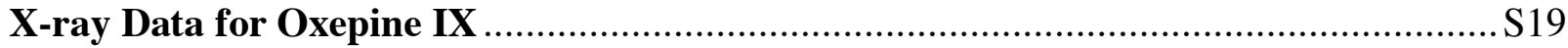

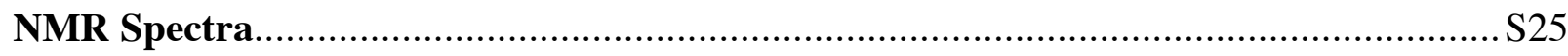

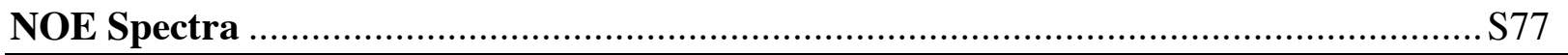




\section{General Information}

Starting materials and reagents were purchased from commercial suppliers and used without further purification except the following: dichloromethane, $\mathrm{Et}_{2} \mathrm{O}$, and benzene were used from a solvent purification system. ${ }^{1} m$-CPBA was washed with phosphate buffer $(\mathrm{pH}=8.0)$ to remove acid and recrystallized from 25:1 $\mathrm{CH}_{2} \mathrm{Cl}_{2}: \mathrm{Et}_{2} \mathrm{O}{ }^{2}$ 3,5-Dinitrobenzoyl chloride was recrystallized from $t$-butyl methyl ether and hexanes. Hexanes and $\mathrm{Et}_{2} \mathrm{O}$ used in chromatography were distilled before use.

All oxygen or moisture sensitive reactions were carried out under $\mathrm{N}_{2}$ atm. in oven-dried $\left(140{ }^{\circ} \mathrm{C}, \geq 4 \mathrm{~h}\right)$ or flame-dried glassware. Air or moisture sensitive liquids were transferred by syringe and were introduced into the reaction flasks through rubber septa under positive $\mathrm{N}_{2}$ pressure. Degassing refers to a flow of dry $\mathrm{N}_{2}$ bubbling through reaction solvent for 15 min. Unless otherwise stated, reactions were stirred with a Teflon-covered stir bar. Concentration refers to the removal of solvent using a Büchi rotary evaporator, followed by use of a vacuum pump at approximately 1 torr. Silica gel chromatography refers to flash chromatography, ${ }^{3}$ and was performed using either Baxter brand silica gel $60 \AA$ (230-400 Mesh ASTM) or EMD brand silica gel $60 \AA$ (particle size 40-63 $\mu \mathrm{M}$ ). Oxepine diastereomers were separated using a Horizon Biotage Flash System (MPLC) with 12-S, 12-M, or 40-M columns.

Proton nuclear magnetic resonance spectra $\left({ }^{1} \mathbf{H}-\mathbf{N M R}\right)$ were measured on either a Varian Unity-300 instrument (300 MHz), a Varian Gemini-400 Instrument (400 MHz), or a Varian Gemini-500 instrument (500 MHz). Chemical shifts are reported in ppm downfield from tetramethylsilane with the solvent reference as the internal standard $\left(\mathrm{CHCl}_{3}: \delta 7.26 \mathrm{ppm}\right)$. Data is reported as follows: chemical shift, multiplicity $(\mathrm{s}=$ singlet, $\mathrm{d}=$ doublet, $\mathrm{t}=$ triplet, $\mathrm{q}=$ quartet, qi $=$ quintet, sex $=$ sextet, $b r=$ broad, $\mathrm{m}=$ multiplet $)$, coupling constants $(\mathrm{Hz})$, and integration. ${ }^{13}$ C-NMR spectra were recorded on either a Varian Unity-300 instrument (75 MHz), a Varian Gemini-400 Instrument (100 MHz), or a Varian Gemini-500 instrument (125 MHz) with complete proton decoupling. Chemical shifts are reported in ppm downfield from tetramethylsilane with the solvent reference as the internal standard $\left(\mathrm{CDCl}_{3}: \delta 77.23 \mathrm{ppm}\right)$. Infrared spectra (IR) were measured on either a Mattson Galaxy Series FTIR 5000 spectrometer or a Nicolet Avatar 360 FT-IR spectrometer and are reported in wave numbers $\left(\mathrm{cm}^{-1}\right)$. Bands are characterized as broad (br), strong (s), medium (m), or weak (w). Elemental analyses (Anal.) were performed by Robertson Microlit Laboratories, Inc., Madison, NJ and are reported in percent atomic abundance. High resolution mass spectral analyses (HRMS) were performed by the Mass Spectrometry Laboratory, University of Illinois at Urbana-Champaign.

X-ray data was collected using a Bruker APEX CCD (charged coupled device) based diffractometer equipped with an LT-2 low temperature apparatus operating at $193 \mathrm{~K}$. A suitable crystal was chosen and mounted on a glass fiber using grease. Data was measured using omega scans of $0.3^{\circ}$ per frame for 45 seconds, such that a hemisphere was collected. A total of 1305 frames were collected with a maximum resolution of $0.90 \AA$. Cell parameters were retrieved

\footnotetext{
${ }^{1}$ Pangborn, A. B.; Giardello, M. A.; Grubbs, R. H.; Rosen, R. K.; Timmers, F. J. Organometallics 1996, 15, 1518.

2 (a) Schwartz, N. N.; Blumbergs, J. H. J. Org. Chem. 1964, 29, 1976. (b) Bortolini, O.; Campestrini, S.; Di Furia, F.; Modena, G. J. Org. Chem. 1987, 52, 5093. (c) Traylor, T. G.; Mikstzal, A. R. J. Am. Chem. Soc. 1987, 109, 2770.

${ }^{3}$ Stille, W. C.; Kahn, M.; Mitra, A. J. Org. Chem. 1978, 43, 2923.
} 
using SMART software ${ }^{4}$ and refined using SAINT on all observed reflections. Data reduction was performed using the SAINT software, ${ }^{5}$ which corrects for Lp and decay. Absorption corrections were applied using SADABS, supplied by George Sheldrick. The structures were solved by the direct method using the SHELXS-97 program $^{6}$ and refined by least squares method on $\mathrm{F}^{2}$, SHELXL-97, ${ }^{7}$ incorporated in SHELXTL-PC V $6.10 .^{8}$

All non-hydrogen atoms are refined anisotropically. Hydrogens were calculated by geometrical methods and refined as a riding model. The crystal used for the diffraction study showed no decomposition during data collection. All drawings are done at $30 \%$ ellipsoids.

NOE 1D experiments were measured on a Varian Gemini-500 instrument (500 MHz) with an indirect probe. Sample size was $5 \mathrm{mg}$ and all samples were degassed to remove oxygen. The number of transients was 512 and the relaxation time was $800 \mathrm{~ms}$. NOESY1D is the Varian implementation of the 1D NOE experiment using pulsed field gradients and selective pulses. The experiment involves a double-pulsed-field-gradient-spin-echo (DPFGSE) pulse sequence that achieves selective spin inversion effectively and cleanly. The NOE values obtained from the experiment were interpreted qualitatively and compared to the relative proton distances to assign the stereochemistry. ${ }^{9}$

\section{Experimental Procedures}

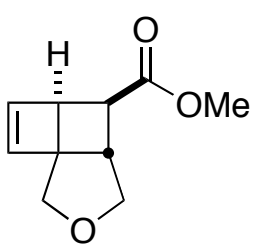

5

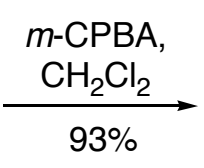

$93 \%$

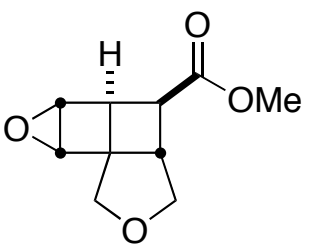

6

A round bottom flask $(25 \mathrm{~mL})$ was charged with cyclobutene $5(348 \mathrm{mg}, 1.90 \mathrm{mmol})$ in $\mathrm{CH}_{2} \mathrm{Cl}_{2}(5.1 \mathrm{~mL})$ and $m$-CPBA $(557 \mathrm{mg}, 3.23 \mathrm{mmol})$. The flask was flushed with $\mathrm{N}_{2}$ and the reaction was stirred at room temperature ( $\mathrm{rt}$ ). The consumption of starting material was monitored by TLC. Upon completion $\left(3 \mathrm{~h}\right.$ ), the reaction was quenched with $\mathrm{Na}_{2} \mathrm{~S}_{2} \mathrm{O}_{3 \text { (sat. aq.) }}$ ( $5 \mathrm{~mL}$ ) and allowed to stir for $1 \mathrm{~h}$. The mixture was diluted with $\mathrm{CH}_{2} \mathrm{Cl}_{2}(20 \mathrm{~mL})$ and the organic layer was separated, washed with $\mathrm{NaHCO}_{3 \text { (sat. aq.) }}$, dried over $\mathrm{MgSO}_{4}$, filtered and concentrated in vacuo. The resulting white solid was purified by silica gel chromatography $\left(1: 1\right.$ hexane:Et $\left.{ }_{2} \mathrm{O}\right)$. Epoxide 6 was obtained in $93 \%$ yield $(346 \mathrm{mg}, 1.76 \mathrm{mmol}$ ) and was recrystallized from $t$-butyl methyl ether to afford crystals suitable for X-ray analysis.

${ }^{4}$ SMART V5.626 (NT) Software for the CCD Detector Systems; Bruker Analytical X-ray Systems, Madison, WI (2001).

${ }^{5}$ SAINT V 5.01 (NT) Software for the CCD Detector Systems; Bruker Analytical X-ray Systems, Madison, WI (2001).

${ }^{6}$ Sheldrick, G. M. SHELXS-90, Program for the Solution of Crystal Structure, University of Göttingen, Germany, 1990.

${ }^{7}$ Sheldrick, G. M. SHELXL-97, Program for the Refinement of Crystal Structure, University of Göttingen, Germany, 1997.

${ }^{8}$ SHELXTL 6.0 (PC-Version), Program Library for Structure Solution and Molecular Graphics; Bruker Analytical X-ray Systems, Madison, WI (1998).

${ }^{9}$ (a) Varian. (b) Stott, K.; Stonehouse, J.; Keeler, J.; Hwang, T. J.; Shaka, A. J. J. Am. Chem. Soc. 1995, 117, 4199. 
Epoxide 6: $\mathbf{m p}=78-79{ }^{\circ} \mathrm{C} .{ }^{1} \mathbf{H}-\mathbf{N M R}\left(400 \mathrm{MHz}, \mathrm{CDCl}_{3}\right): \delta 4.06(\mathrm{~m}, 2 \mathrm{H}), 3.97(\mathrm{~d}, J=9.6 \mathrm{~Hz}$, $1 \mathrm{H}), 3.86(\mathrm{~d}, J=10.4 \mathrm{~Hz}, 1 \mathrm{H}), 3.73(\mathrm{~m}, 1 \mathrm{H}) 3.73(\mathrm{~s}, 3 \mathrm{H}), 3.45(\mathrm{~d}, J=10.4 \mathrm{~Hz}, 1 \mathrm{H}), 3.21$, (dd, $J$ $=8.0,5.2 \mathrm{~Hz}, 1 \mathrm{H}), 3.06,(\mathrm{t}, J=5.2 \mathrm{~Hz}, 1 \mathrm{H}), 2.79,(\mathrm{dd}, J=8.0,3.6 \mathrm{~Hz}, 1 \mathrm{H}) .{ }^{13} \mathbf{C}-\mathbf{N M R}(100$ $\left.\mathrm{MHz}, \mathrm{CDCl}_{3}\right): \delta 172.8,73.4,69.7,60.4,54.4,52.3,51.8,46.6,42.1,41.4$. IR (KBr pellet) 3428 (br), 2987 (m), 2965 (m), 2920 (m), 2861 (m), 1725 (s), 1440 (m), 1341 (m), 1312 (m), 1264 (s), $1093(\mathrm{~s}), 822(\mathrm{~m}) \mathrm{cm}^{-1}$. Anal. Calcd for $\mathrm{C}_{10} \mathrm{H}_{12} \mathrm{O}_{4}:$ C, 61.22; H, 6.16. Found: C, 61.40; H, 6.08.

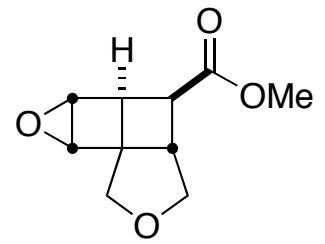

6

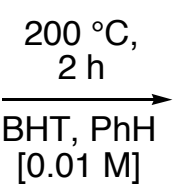

$[0.01 \mathrm{M}]$

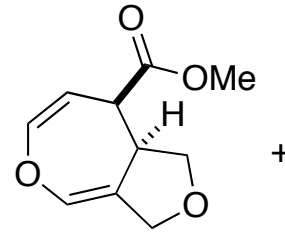

$7(46 \%)$

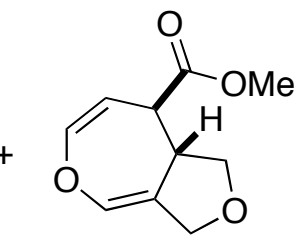

$8(35 \%)$

A degassed solution of $6(68.0 \mathrm{mg}, 0.347 \mathrm{mmol})$ and BHT $(115 \mathrm{mg}, 0.521 \mathrm{mmol})$ in benzene $(34.7 \mathrm{~mL})$ in a heavy-wall sealed tube $(150 \mathrm{~mL})$ was placed in a silicone oil bath and heated to $200{ }^{\circ} \mathrm{C}$. The reaction was monitored by TLC and stopped upon consumption of starting material $(2 \mathrm{~h})$. Concentration in vacuo, followed by filtration through basic alumina ( $1 \mathrm{~cm}$ plug) with hexanes (to separate BHT) followed by $\mathrm{Et}_{2} \mathrm{O}$ afforded two diastereomers as a colorless oil. Medium pressure liquid chromatography (10-30\% hexane: $\left.\mathrm{Et}_{2} \mathrm{O}\right)$ with a base-washed column (1\% pyridine in EtOAc) was used to separate the diastereomers. Oxepine 7 was obtained as a colorless oil in $46 \%$ yield (31 mg, $0.16 \mathrm{mmol}$ ) and oxepine 8 was obtained as a colorless oil in $35 \%$ yield ( $24 \mathrm{mg}, 0.12 \mathrm{mmol})$.

Oxepine 7: ${ }^{1} \mathrm{H}-\mathrm{NMR}\left(400 \mathrm{MHz}, \mathrm{CDCl}_{3}\right): \delta 6.43(\mathrm{~s}, 1 \mathrm{H}), 6.33(\mathrm{~d}, J=8.0 \mathrm{~Hz}, 1 \mathrm{H}), 4.77(\mathrm{t}, J=$ $8.0 \mathrm{~Hz}, 1 \mathrm{H}), 4.48(\mathrm{~d}, J=11.6 \mathrm{~Hz}, 1 \mathrm{H}), 4.34(\mathrm{~d}, J=11.6 \mathrm{~Hz}, 1 \mathrm{H}), 4.04(\mathrm{t}, J=8.4 \mathrm{~Hz}, 1 \mathrm{H}), 3.74$ $(\mathrm{dd}, J=8.8,6.8 \mathrm{~Hz}, 1 \mathrm{H}), 3.70(\mathrm{~s}, 3 \mathrm{H}), 3.47(\mathrm{dd}, J=8.0,3.6 \mathrm{~Hz}, 1 \mathrm{H}), 3.34(\mathrm{~m}, 1 \mathrm{H}) .{ }^{13} \mathbf{C}-\mathbf{N M R}$ $\left(100 \mathrm{MHz}, \mathrm{CDCl}_{3}\right): \delta 171.9,144.5,134.1,121.3,102.2,72.6,70.7,52.5,45.9,44.6 . \quad$ IR $(\mathrm{NaCl}$ thin film): 3055 (w), 2924 (w), 2853 (w), 2361(w), 2341(w), 1740 (m), 1651 (w), 1266 (s), 1196 (w), 1152 (m), 738 (s), 704 (w) cm $\mathrm{cm}^{-1}$. EI HRMS: Calcd for $\mathrm{C}_{10} \mathrm{H}_{12} \mathrm{O}_{4}\left({ }^{+} \mathrm{M}\right)$ : 196.0736; Found: 196.0732 .

Oxepine 8: ${ }^{1} \mathrm{H}-\mathrm{NMR}\left(400 \mathrm{MHz}, \mathrm{CDCl}_{3}\right): \delta 6.38(\mathrm{~s}, 1 \mathrm{H}), 6.23(\mathrm{~d}, J=8.0 \mathrm{~Hz}, 1 \mathrm{H}), 4.82(\mathrm{~d}, J=$ 8.0, 1H), $4.33(\mathrm{~m}, 2 \mathrm{H}), 4.15(\mathrm{~m}, 1 \mathrm{H}), 3.74(\mathrm{~s}, 3 \mathrm{H}), 3.61(\mathrm{~m}, 1 \mathrm{H}), 3.34(\mathrm{~m}, 2 \mathrm{H}),{ }^{13} \mathbf{C}-\mathbf{N M R}(100$ $\left.\mathrm{MHz}, \mathrm{CDCl}_{3}\right): \delta 172.6,142.5,134.9,120.5,104.5,73.8,70.3,52.5,46.6,45.0 . \quad$ IR $(\mathrm{NaCl}$ thin film): 2954 (m), 2849 (m), 1737 (s), $1695(\mathrm{~m}), 1656(\mathrm{~m}), 1435$ (w), $1361(\mathrm{w}), 1302(\mathrm{w}), 1156$ (m) $\mathrm{cm}^{-1}$. Anal. Calcd for $\mathrm{C}_{10} \mathrm{H}_{12} \mathrm{O}_{4}: \mathrm{C}, 61.22 ; \mathrm{H}, 6.16$. Found: C, 61.35; H, 6.40.

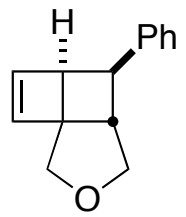

I

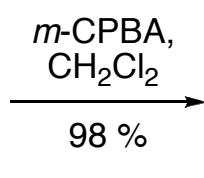

A round bottom flask ( $5 \mathrm{~mL}$ ) was charged with cyclobutene $\mathbf{I}(50 \mathrm{mg}, 0.25 \mathrm{mmol})$ in $\mathrm{CH}_{2} \mathrm{Cl}_{2}$ $(0.68 \mathrm{~mL})$ and $m$-CPBA $(65 \mathrm{mg}, 0.38 \mathrm{mmol})$. The flask was flushed with $\mathrm{N}_{2}$ and allowed to stir at $\mathrm{rt}$. The consumption of starting material was monitored by TLC. Upon completion $(1 \mathrm{~h})$, the reaction was quenched with $\mathrm{Na}_{2} \mathrm{~S}_{2} \mathrm{O}_{3 \text { (sat. aq.) }}(1.5 \mathrm{~mL})$, diluted with $\mathrm{CH}_{2} \mathrm{Cl}_{2}(1.5 \mathrm{~mL})$ and allowed to 
stir for $1 \mathrm{~h}$. The mixture was diluted with $\mathrm{CH}_{2} \mathrm{Cl}_{2}(20 \mathrm{~mL})$ and the organic layer was separated, washed with $\mathrm{NaHCO}_{3 \text { (sat. aq.) }}$, dried over $\mathrm{MgSO}_{4}$, filtered and concentrated in vacuo. The resulting oil was purified by silica gel chromatography $\left(1: 1\right.$ hexane: $\left.\mathrm{Et}_{2} \mathrm{O}\right)$. Epoxide 9 was obtained in $98 \%$ yield as a light yellow oil (53 $\mathrm{mg}, 0.25 \mathrm{mmol})$.

Epoxide 9: ${ }^{1} \mathbf{H}-\mathbf{N M R}\left(400 \mathrm{MHz}, \mathrm{CDCl}_{3}\right): \delta 7.35(\mathrm{t}, J=8.0 \mathrm{~Hz}, 2 \mathrm{H}), 7.23(\mathrm{~m}, 3 \mathrm{H}), 4.03(\mathrm{~m}, 2 \mathrm{H})$, $3.95(\mathrm{~d}, J=10.4 \mathrm{~Hz}, 1 \mathrm{H}), 3.82(\mathrm{dd}, J=10.0,6.8 \mathrm{~Hz}, 1 \mathrm{H}), 3.75(\mathrm{~s}, 1 \mathrm{H}), 3.69(\mathrm{t}, J=7.6 \mathrm{~Hz}, 1 \mathrm{H})$, $3.53(\mathrm{~d}, J=10.4 \mathrm{~Hz}, 1 \mathrm{H}), 3.09(\mathrm{t}, J=6.0 \mathrm{~Hz}, 1 \mathrm{H}), 2.99(\mathrm{dd}, J=8.4,4.0 \mathrm{~Hz}, 1 \mathrm{H}) .{ }^{13} \mathbf{C}-\mathbf{N M R}$ $\left(100 \mathrm{MHz}, \mathrm{CDCl}_{3}\right): \delta 140.6,128.2,126.3,126.0,73.4,69.4,59.2,54.2,51.4,48.9,43.7,42.3$. IR (NaCl thin film): 3056 (w), 2961 (w), 2851 (w), 2360 (w), $1266(\mathrm{w}), 736(\mathrm{~m}), 701(\mathrm{~m}) \mathrm{cm}^{-1}$. Anal. Calcd for $\mathrm{C}_{14} \mathrm{H}_{14} \mathrm{O}_{2}$ : C, 78.48; H, 6.59. Found C, 78.27; H, 6.87.

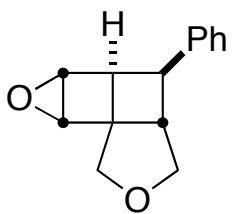

9

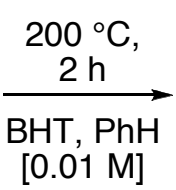

$[0.01 \mathrm{M}]$

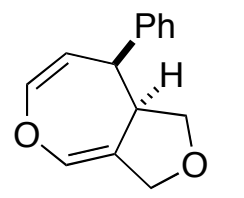

$10(53 \%)$

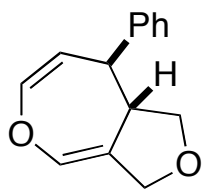

$11(15 \%)$

A degassed solution of epoxide 9 (178 $\mathrm{mg}, 0.831 \mathrm{mmol})$ and BHT (275 $\mathrm{mg}, 1.25 \mathrm{mmol})$ in benzene $(83.1 \mathrm{~mL})$ in a heavy-wall sealed tube $(350 \mathrm{~mL})$ was placed in a silicone oil bath and heated to $200{ }^{\circ} \mathrm{C}$. The reaction was monitored by TLC and stopped upon consumption of starting material $(2.5 \mathrm{~h})$. Concentration in vacuo, followed by filtration through basic alumina (1 $\mathrm{cm}$ plug) with hexanes (to separate $\mathrm{BHT}$ ) followed by $\mathrm{Et}_{2} \mathrm{O}$ afforded two diastereomers as a colorless oil. Medium pressure liquid chromatography (5-10\% $\mathrm{Et}_{2} \mathrm{O}$ :hexane) with a base-washed column (1\% pyridine in EtOAc) was used to separate the diastereomers. Oxepine 10 was obtained as a colorless oil (becomes solid at colder temperatures) in 53\% yield (94.8 mg, 0.393 $\mathrm{mmol}$ ) and oxepine $\mathbf{1 1}$ was obtained as a colorless oil (becomes solid at colder temperatures) in $15 \%$ yield $(27.5 \mathrm{mg}, 0.128 \mathrm{mmol})$.

Oxepine 10: $\mathbf{m p}=46-48{ }^{\circ} \mathrm{C} .{ }^{1} \mathbf{H}-\mathbf{N M R}\left(400 \mathrm{MHz}, \mathrm{CDCl}_{3}\right): \delta 7.26(\mathrm{~m}, 5 \mathrm{H}), 6.49(\mathrm{~s}, 1 \mathrm{H}), 6.34$ $(\mathrm{d}, J=8.0 \mathrm{~Hz}, 1 \mathrm{H}), 4.92(\mathrm{t}, J=8.0 \mathrm{~Hz}, 1 \mathrm{H}), 4.19(\mathrm{~d}, J=11.6 \mathrm{~Hz}, 1 \mathrm{H}), 3.97(\mathrm{t}, J=8.0 \mathrm{~Hz}, 1 \mathrm{H})$, $3.90(\mathrm{dt}, J=11.6,2.0 \mathrm{~Hz}, 1 \mathrm{H}), 3.72(\mathrm{dd}, J=8.0,4.0 \mathrm{~Hz}, 1 \mathrm{H}), 3.64(\mathrm{~m}, 1 \mathrm{H}), 3.41(\mathrm{t}, J=8.0 \mathrm{~Hz}$, 1H). ${ }^{13}$ C-NMR $\left(100 \mathrm{MHz}, \mathrm{CDCl}_{3}\right): \delta 142.4,141.4,134.3,129.8,128.2,127.0,121.6,108.9$, 71.9, 70.2, 46.9, 46.0. IR (KBr pellet): 3020 (m), 2960 (m), $2856(\mathrm{~m}), 2813(\mathrm{~m}), 2361(\mathrm{w})$, 1696 (m), 1636 (s), 1304 (s), 1152 (s) $\mathrm{cm}^{-1}$. Anal. Calcd for $\mathrm{C}_{14} \mathrm{H}_{14} \mathrm{O}_{2}$ : C, 78.48; H, 6.59. Found C, 78.32; H, 6.47.

Oxepine 11: $\mathbf{m p}=60-62{ }^{\circ} \mathrm{C} .{ }^{1} \mathbf{H}-\mathbf{N M R}\left(400 \mathrm{MHz}, \mathrm{CDCl}_{3}\right): \delta 7.33(\mathrm{~m}, 2 \mathrm{H}), 7.24(\mathrm{~m}, 1 \mathrm{H}), 7.18$ $(\mathrm{m}, 2 \mathrm{H}), 6.45(\mathrm{~s}, 1 \mathrm{H}), 6.23(\mathrm{dd}, J=8.0,2.8 \mathrm{~Hz}, 1 \mathrm{H}), 4.71(\mathrm{dd}, J=8.0,2.8 \mathrm{~Hz}, 1 \mathrm{H}), 4.39(\mathrm{~m}, 2 \mathrm{H})$, $3.64(\mathrm{dd}, J=9.2,7.6 \mathrm{~Hz}, 1 \mathrm{H}), 3.49(\mathrm{~m}, 2 \mathrm{H}), 3.28(\mathrm{~m}, 1 \mathrm{H}) .{ }^{13} \mathbf{C}-\mathbf{N M R}\left(100 \mathrm{MHz}, \mathrm{CDCl}_{3}\right): \delta$ 144.0, 141.0, 135.0, 128.9, 127.6, 127.0, 122.0, 111.4, 73.8, 70.7, 49.8, 47.2. IR (KBr pellet): 3036 (w), 2998 (m), 2905 (m), 2845 (s), 1702 (s), 1653 (s), 1299 (s), 1162 (s). EI HRMS: Calcd for $\mathrm{C}_{14} \mathrm{H}_{14} \mathrm{O}_{2}(+\mathrm{M})$ : 214.0994; Found: 214.0989 . 


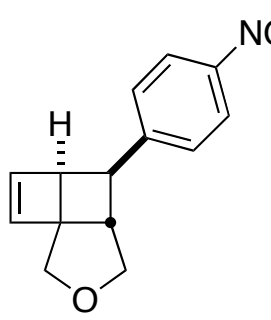

II

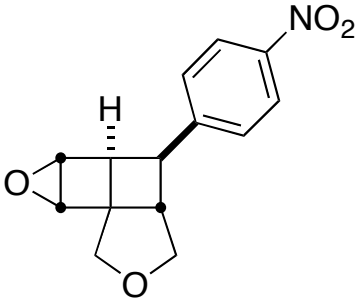

12

A round bottom flask $(5 \mathrm{~mL})$ was charged with cyclobutene $\mathbf{I I}(52.3 \mathrm{mg}, 0.215 \mathrm{mmol})$ in $\mathrm{CH}_{2} \mathrm{Cl}_{2}(0.57 \mathrm{~mL})$ and $m$-CPBA $(72.5 \mathrm{mg}, 0.430 \mathrm{mmol})$. The flask was flushed with $\mathrm{N}_{2}$ and allowed to stir at rt. The consumption of starting material was monitored by TLC. Upon completion $(4 \mathrm{~h})$, the reaction was quenched with $\mathrm{Na}_{2} \mathrm{~S}_{2} \mathrm{O}_{3 \text { (sat. aq.) }}(1.5 \mathrm{~mL})$, diluted with $\mathrm{CH}_{2} \mathrm{Cl}_{2}$ (1.5 mL) and allowed to stir for $1 \mathrm{~h}$. The mixture was diluted with $\mathrm{CH}_{2} \mathrm{Cl}_{2}(20 \mathrm{~mL})$ and the organic layer was separated, washed with $\mathrm{NaHCO}_{3 \text { (sat. aq.) }}$, dried over $\mathrm{MgSO}_{4}$, filtered and concen-

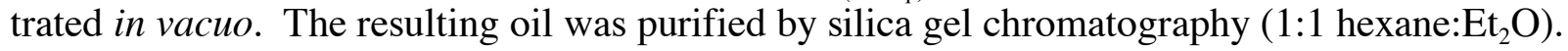
Epoxide 12 was obtained in $97 \%$ yield as a light yellow solid (53 mg, $0.20 \mathrm{mmol})$.

Epoxide 12: $\mathbf{m p}=146-148{ }^{\circ} \mathrm{C} .{ }^{1} \mathbf{H}-\mathbf{N M R}\left(400 \mathrm{MHz}, \mathrm{CDCl}_{3}\right): \delta 8.22(\mathrm{~d}, J=8.8 \mathrm{~Hz}, 2 \mathrm{H}), 7.37$ $(\mathrm{d}, J=8.8 \mathrm{~Hz}, 2 \mathrm{H}), 4.06(\mathrm{~m}, 2 \mathrm{H}), 3.97(\mathrm{~d}, J=10.4 \mathrm{~Hz}, 1 \mathrm{H}), 3.85(\mathrm{dd}, J=10.0,6.4 \mathrm{~Hz}, 1 \mathrm{H}), 3.78$ $(\mathrm{t}, J=7.2 \mathrm{~Hz}, 1 \mathrm{H}), 3.68(\mathrm{~d}, 1.6 \mathrm{~Hz}, 1 \mathrm{H}), 3.54(\mathrm{~d}, 10.8 \mathrm{~Hz}, 1 \mathrm{H}), 3.13(\mathrm{t}, J=6.4 \mathrm{~Hz}, 1 \mathrm{H}), 3.04(\mathrm{dd}$, $J=8.0,3.6 \mathrm{~Hz}, 1 \mathrm{H}) .{ }^{13} \mathrm{C}-\mathbf{N M R}\left(100 \mathrm{MHz}, \mathrm{CDCl}_{3}\right): \delta 148.9,146.8,127.7,124.1,73.3,69.9$, 60.0, 54.7, 51.6, 49.3, 44.3, 42.7. IR (KBr pellet): 3440 (br), 3072 (w), 2969 (w), 2923 (w),

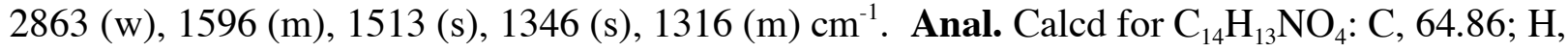
5.05; N, 5.40. Found: C, 65.05; H, 5.30; N, 5.12.

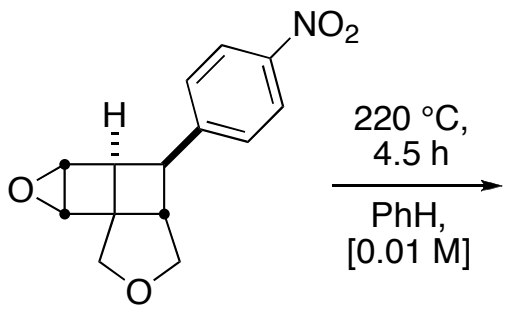

12

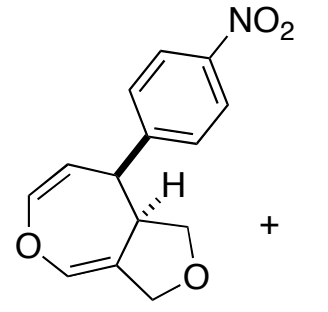

$13(52 \%)$

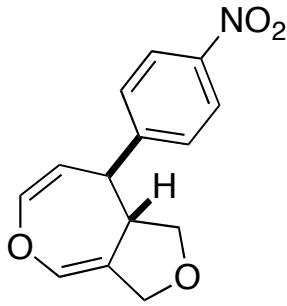

$14(18 \%)$

A degassed solution of $12(23.7 \mathrm{mg}, 0.0914 \mathrm{mmol})$ and in benzene $(9.1 \mathrm{~mL}, 10 \mathrm{mM})$ in a heavy-wall sealed tube $(35 \mathrm{~mL})$ was placed in a silicone oil bath and heated to $220{ }^{\circ} \mathrm{C}$. The reaction was monitored by TLC and stopped upon consumption of starting material $(4.5 \mathrm{~h})$. Concentration in vacuo, followed by medium pressure liquid chromatography $(10-20 \%$ $\mathrm{Et}_{2} \mathrm{O}$ :hexane) with a base-washed column (1\% pyridine in EtOAc) was used to separate the diastereomers. Oxepine 13 was obtained as a yellow solid in 52\% yield (12.4 mg, $0.0478 \mathrm{mmol})$ and oxepine 14 was obtained as a yellow solid in $18 \%$ yield $(4.3 \mathrm{mg}, 0.018 \mathrm{mmol})$.

Oxepine 13: $\mathbf{m p}=103-104{ }^{\circ} \mathrm{C} .{ }^{1} \mathbf{H}-\mathbf{N M R}\left(400 \mathrm{MHz}, \mathrm{CDCl}_{3}\right): \delta 8.15(\mathrm{~d}, J=8.0 \mathrm{~Hz}, 2 \mathrm{H}), 7.40$ $(\mathrm{d}, J=8.0 \mathrm{~Hz}, 2 \mathrm{H}), 6.55(\mathrm{~s}, 1 \mathrm{H}), 6.40(\mathrm{~d}, J=8.0 \mathrm{~Hz}, 1 \mathrm{H}), 4.90(\mathrm{t}, J=8.0 \mathrm{~Hz}, 1 \mathrm{H}), 4.15(\mathrm{~d}, J=$ $11.6 \mathrm{~Hz}, 1 \mathrm{H}), 3.95(\mathrm{~m}, 2 \mathrm{H}), 3.87(\mathrm{dd}, J=7.6,4.0 \mathrm{~Hz}, 1 \mathrm{H}), 3.67(\mathrm{~m}, 1 \mathrm{H}), 3.46(\mathrm{dd}, J=9.2,6.0$ $\mathrm{Hz}, 1 \mathrm{H}) .{ }^{13} \mathrm{C}-\mathbf{N M R}\left(100 \mathrm{MHz}, \mathrm{CDCl}_{3}\right): \delta 149.2,143.2,134.8,130.4,128.4,123.3,121.2,107.4$, 71.8, 70.9, 46.7, 46.5. IR (KBr pellet): 3440 (br), 3075 (m), 3053 (m), 3029 (s), 2942 (s), 2903 (s), 2849 (s), 1648 (w), 1449 (s), 1351 (m), 1153 (w), 1020 (m), 910 (m). EI HRMS: Calcd for $\mathrm{C}_{14} \mathrm{H}_{13} \mathrm{NO}_{4}\left({ }^{+} \mathrm{M}\right)$ : 259.0845; Found: 259.0840 . 
Oxepine 14: $\mathbf{m p}=130-132{ }^{\circ} \mathrm{C} .{ }^{1} \mathbf{H}-\mathbf{N M R}\left(400 \mathrm{MHz}, \mathrm{CDCl}_{3}\right): \delta 8.21(\mathrm{~d}, J=8.0 \mathrm{~Hz}, 2 \mathrm{H}), 7.36$ $(\mathrm{d}, J=8.0 \mathrm{~Hz}, 2 \mathrm{H}), 6.48(\mathrm{~d}, J=2.0 \mathrm{~Hz}, 1 \mathrm{H}), 6.28(\mathrm{dd}, J=8.0,2.8 \mathrm{~Hz}, 1 \mathrm{H}), 4.61(\mathrm{dd}, J=8.0,2.4$ $\mathrm{Hz}, 1 \mathrm{H}), 4.40(\mathrm{t}, J=1.6 \mathrm{~Hz}, 2 \mathrm{H}), 3.64(\mathrm{~m}, 2 \mathrm{H}), 3.43(\mathrm{dd}, J=8.8,6.8 \mathrm{~Hz}, 1 \mathrm{H}), 3.28(\mathrm{~m}, 1 \mathrm{H})$.

${ }^{13}$ C-NMR $\left(100 \mathrm{MHz}, \mathrm{CDCl}_{3}\right): \delta 151.0,141.9,135.3,128.6,124.3,121.9,109.3,73.4,70.6,49.5$, 46.9. IR (KBr pellet): 3466 (br), 3061 (w), 3027 (s), 2919 (s), 2853 (w), 1693 (w), 1649 (m), 1598 (w), 1519 (s), 1349 (s), 1300 (m), 1154 (m), 936 (m), 753 (m). EI HRMS: Calcd for $\mathrm{C}_{14} \mathrm{H}_{13} \mathrm{NO}_{4}\left({ }^{+} \mathrm{M}\right): 259.0845$; Found: 259.0849 .

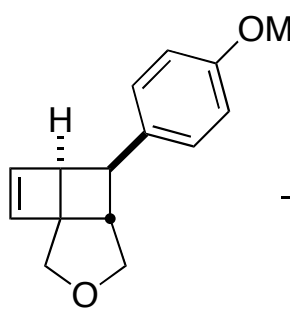

III

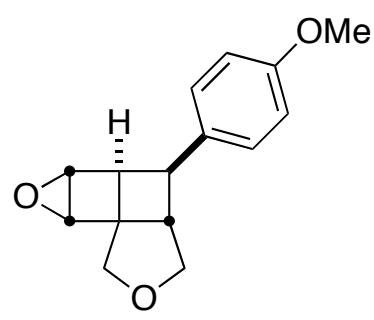

15

A round bottom flask $(5 \mathrm{~mL})$ was charged with cyclobutene III (106 mg, $0.464 \mathrm{mmol})$ in $\mathrm{CH}_{2} \mathrm{Cl}_{2}(1.2 \mathrm{~mL})$ and $m$-CPBA $(148 \mathrm{mg}, 0.858 \mathrm{mmol})$. The flask was flushed with $\mathrm{N}_{2}$ and allowed to stir at rt. The consumption of starting material was monitored by TLC. Upon completion ( $2 \mathrm{~h}$ ), the reaction was quenched with $\mathrm{Na}_{2} \mathrm{~S}_{2} \mathrm{O}_{3 \text { (sat. aq.) }}\left(1.5 \mathrm{~mL}\right.$ ), diluted with $\mathrm{CH}_{2} \mathrm{Cl}_{2}$ $(1.5 \mathrm{~mL})$ and allowed to stir for $1 \mathrm{~h}$. The mixture was diluted with $\mathrm{CH}_{2} \mathrm{Cl}_{2}(20 \mathrm{~mL})$ and the organic layer was separated, washed with $\mathrm{NaHCO}_{3 \text { (sat aq.) }}$, dried over $\mathrm{MgSO}_{4}$, filtered and concentrated in vacuo. The resulting oil was purified by silica gel chromatography (1:1 hexane: $\left.\mathrm{Et}_{2} \mathrm{O}\right)$. Epoxide 15 was obtained in $97 \%$ yield as a white solid $(109 \mathrm{mg}, 0.446 \mathrm{mmol})$.

Epoxide 15: $\mathbf{~ m p}=82-84^{\circ} \mathrm{C} .{ }^{1} \mathrm{H}-\mathrm{NMR}\left(400 \mathrm{MHz}, \mathrm{CDCl}_{3}\right): \delta 7.13(\mathrm{~d}, J=8.4 \mathrm{~Hz}, 2 \mathrm{H}), 6.88(\mathrm{~d}$, $J=8.4 \mathrm{~Hz}, 2 \mathrm{H}), 4.04(\mathrm{dd}, J=3.6,2.0 \mathrm{~Hz}, 1 \mathrm{H}), 4.02(\mathrm{~d}, J=12.0,1 \mathrm{H}), 3.93(\mathrm{~d}, J=10.0 \mathrm{~Hz}, 1 \mathrm{H})$, $3.80(\mathrm{~m}, 1 \mathrm{H}), 3.80(\mathrm{~s}, 3 \mathrm{H}), 3.75(\mathrm{~d}, J=2.0 \mathrm{~Hz}, 1 \mathrm{H}), 3.62(\mathrm{t}, J=6.8 \mathrm{~Hz}, 1 \mathrm{H}), 3.52(\mathrm{~d}, J=10.0$ $\mathrm{Hz}, 1 \mathrm{H}), 3.04(\mathrm{t}, J=6.0 \mathrm{~Hz}, 1 \mathrm{H}), 2.95,(\mathrm{dd}, J=8.0,3.6 \mathrm{~Hz}, 1 \mathrm{H}) .{ }^{13} \mathbf{C}-\mathbf{N M R}\left(100 \mathrm{MHz}, \mathrm{CDCl}_{3}\right)$ : $\delta$ 157.8, 132.9, 127.5, 113.8, 73.6, 69.6, 59.2, 55.3, 54.5, 51.6, 49.2, 44.1, 41.9. IR (KBr pellet): $3047(\mathrm{w}), 3025(\mathrm{w}), 2954(\mathrm{~m}), 2856(\mathrm{~m}), 1609(\mathrm{~m}), 1511(\mathrm{~s}), 1250(\mathrm{~s}) \mathrm{cm}^{-1}$. Anal. Calcd for $\mathrm{C}_{15} \mathrm{H}_{16} \mathrm{O}_{3}:$ C, 73.75; H, 6.60. Found: C, 73.59; H, 6.42.

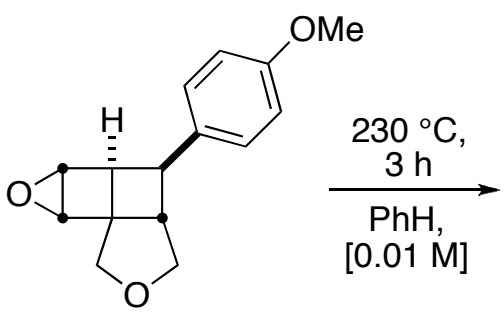

15

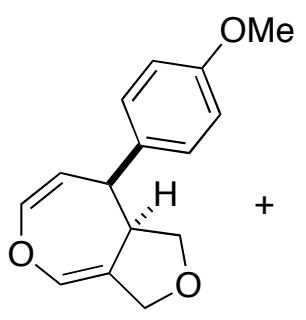

$16(48 \%)$

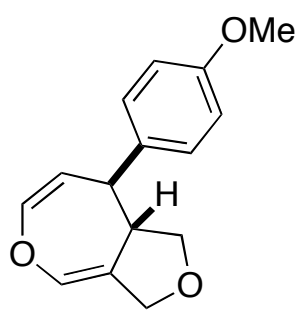

$17(23 \%)$

A degassed solution of $\mathbf{1 5}(18 \mathrm{mg}, 0.074 \mathrm{mmol})$ and in benzene $(7.4 \mathrm{~mL}, 10.0 \mathrm{mM})$ in a heavy-wall sealed tube $(35 \mathrm{~mL})$ was placed in a silicone oil bath and heated to $230{ }^{\circ} \mathrm{C}$. The reaction was monitored by TLC and stopped upon consumption of starting material $(3 \mathrm{~h})$. Concentration in vacuo, followed by medium pressure liquid chromatography (10-20\% $\mathrm{Et}_{2} \mathrm{O}$ :hexane) with a base-washed column (1\% pyridine in EtOAc) was used to separate the 
diastereomers. Oxepine 16 was obtained as a colorless oil in $48 \%$ yield $(8.6 \mathrm{mg}, 0.035 \mathrm{mmol})$ and oxepine 17 was obtained as a colorless oil in $23 \%$ yield $(4.2 \mathrm{mg}, 0.017 \mathrm{mmol}$ ).

Oxepine 16: ${ }^{1} \mathbf{H}-\mathbf{N M R}\left(400 \mathrm{MHz}, \mathrm{CDCl}_{3}\right): \delta 7.15(\mathrm{~d}, J=6.8 \mathrm{~Hz}, 2 \mathrm{H}), 6.83(\mathrm{~d}, J=6.8 \mathrm{~Hz}, 2 \mathrm{H})$, $6.48(\mathrm{~d}, J=2.0 \mathrm{~Hz}, 1 \mathrm{H}), 6.32(\mathrm{~d}, J=6.4 \mathrm{~Hz}), 4.91(\mathrm{t}, J=6.4 \mathrm{~Hz}, 1 \mathrm{H}), 4.18(\mathrm{~d}, J=9.6 \mathrm{~Hz}, 1 \mathrm{H})$, $3.96(\mathrm{t}, J=6.4 \mathrm{~Hz}, 1 \mathrm{H}), 3.92(\mathrm{dt}, J=9.2,1.6 \mathrm{~Hz}, 1 \mathrm{H}), 3.79(\mathrm{~s}, 3 \mathrm{H}), 3.64(\mathrm{dd}, J=6.4,3.2 \mathrm{~Hz}$, $1 \mathrm{H}), 3.62(\mathrm{~m}, 1 \mathrm{H}), 3.42(\mathrm{dd}, J=6.8,5.6 \mathrm{~Hz}, 1 \mathrm{H}) .{ }^{13} \mathbf{C}-\mathbf{N M R}\left(100 \mathrm{MHz}, \mathrm{CDCl}_{3}\right): \delta 158.6,142.3$, 134.3, 133.6, 130.8, 121.8, 113.6, 109.3, 71.9, 70.1, 55.4, 46.8, 45.1. IR (NaCl thin film): 3080 (w), 3020 (w), 2949 (m), 2927 (s), 2840 (m), 1702 (m), 1653 (m), 1609 (m), 1511 (s), 1250 (m), $1146(\mathrm{~m}) \mathrm{cm}^{-1}$. EI HRMS: Calcd for $\mathrm{C}_{15} \mathrm{H}_{16} \mathrm{O}_{3}\left({ }^{+} \mathrm{M}\right)$ : 244.1099; Found: 244.1103.

Oxepine 17: ${ }^{1} \mathrm{H}-\mathrm{NMR}\left(400 \mathrm{MHz}, \mathrm{CDCl}_{3}\right): \delta 7.09(\mathrm{~d}, J=6.8 \mathrm{~Hz}, 2 \mathrm{H}), 6.86(\mathrm{~d}, J=6.8 \mathrm{~Hz}, 2 \mathrm{H})$, $6.44(\mathrm{~s}, 1 \mathrm{H}), 6.20(\mathrm{dd}, J=6.0,2.0 \mathrm{~Hz}, 1 \mathrm{H}), 4.68(\mathrm{dd}, J=6.4,2.0 \mathrm{~Hz}, 1 \mathrm{H}), 4.38(\mathrm{~s}, 2 \mathrm{H}), 3.80$ (s, $3 \mathrm{H}), 3.65(\mathrm{t}, J=6.4 \mathrm{~Hz}, 1 \mathrm{H}), 3.46(\mathrm{~m}, 1 \mathrm{H}), 3.23(\mathrm{~m}, 1 \mathrm{H}),{ }^{13} \mathbf{C}-\mathbf{N M R}\left(100 \mathrm{MHz} \mathrm{CDCl}_{3}\right): \delta$ $158.6,141.0,136.5,135.1,128.6,122.2,114.3,111.8,73.9,70.7,55.5,49.9,46.3$. IR $(\mathrm{NaCl}$ thin film): 3031 (w), 2954 (m), 2829 (m), 1696 (m), 1642 (s), 1511 (s), 1304 (s), 1255 (s), 1146 (s), $814(\mathrm{~s}) \mathrm{cm}^{-1}$. EI HRMS: Calcd for $\mathrm{C}_{15} \mathrm{H}_{16} \mathrm{O}_{3}\left({ }^{+} \mathrm{M}\right): 244.1099$; Found: 244.1102.

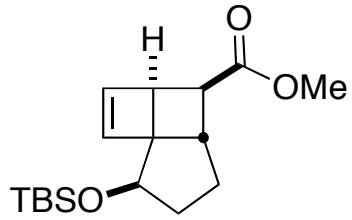

IV

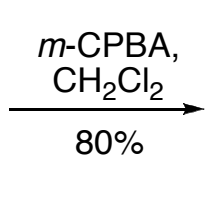

TBSO

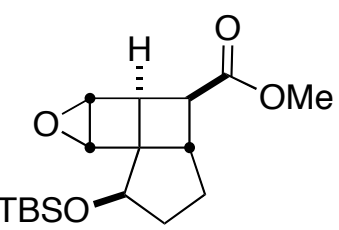

18

A round bottom flask $(10 \mathrm{~mL})$ was charged with cyclobutene $\mathbf{I V}(270 \mathrm{mg}, 0.875 \mathrm{mmol})$ in $\mathrm{CH}_{2} \mathrm{Cl}_{2}(2.2 \mathrm{~mL})$ and $m$-CPBA $(206 \mathrm{mg}, 1.19 \mathrm{mmol})$. The flask was flushed with $\mathrm{N}_{2}$ and allowed to stir at rt. The consumption of starting material was monitored by TLC. Upon completion (3 h), the reaction was quenched with $\mathrm{Na}_{2} \mathrm{~S}_{2} \mathrm{O}_{3 \text { (sat. aq.) }}(3.0 \mathrm{~mL})$, diluted with $\mathrm{CH}_{2} \mathrm{Cl}_{2}(3.0 \mathrm{~mL})$ and allowed to stir for $1 \mathrm{~h}$. The mixture was diluted with $\mathrm{CH}_{2} \mathrm{Cl}_{2}(30 \mathrm{~mL})$ and the organic layer was separated, washed with $\mathrm{NaHCO}_{3 \text { (sat. aq.) }}$, dried over $\mathrm{MgSO}_{4}$, filtered and concentrated in vacuo. The resulting oil was purified by silica gel chromatography $\left(2: 1\right.$ hexane:Et $\left.{ }_{2} \mathrm{O}\right)$. Epoxide $\mathbf{1 8}$ was obtained in $80 \%$ yield as a colorless oil $(227 \mathrm{mg}, 0.700 \mathrm{mmol})$.

Epoxide 18: ${ }^{1} \mathbf{H}-\mathrm{NMR}\left(400 \mathrm{MHz}, \mathrm{CDCl}_{3}\right)$ : $\delta 4.05(\mathrm{~d}, J=2.4 \mathrm{~Hz}, 1 \mathrm{H}), 3.94(\mathrm{~m}, 2 \mathrm{H}), 3.71(\mathrm{~s}$, $3 \mathrm{H}), 2.88(\mathrm{~m}, 2 \mathrm{H}), 2.51(\mathrm{dd}, J=8.0,4.0 \mathrm{~Hz}, 1 \mathrm{H}), 2.10(\mathrm{~m}, 1 \mathrm{H}), 1.68(\mathrm{~m}, 3 \mathrm{H}), 0.88(\mathrm{~s}, 9 \mathrm{H}), 0.08$ $(\mathrm{d}, J=14.8,6 \mathrm{H}) .{ }^{13} \mathrm{C}-\mathrm{NMR}\left(100 \mathrm{MHz}, \mathrm{CDCl}_{3}\right): \delta 173.2,71.2,63.9,53.7,52.0,44.0,41.0,39.3$, 34.7, 29.4, 26.0, 18.4, -4.6. IR (NaCl thin film): 3028 (w), 2954 (s), $2896(\mathrm{~m}), 2858(\mathrm{~m}), 1737$ (s), $1472(\mathrm{w}), 1436(\mathrm{w}), 1256(\mathrm{~s}), 1175(\mathrm{~m}), 1076(\mathrm{~m}), 1052(\mathrm{~m}), 1002(\mathrm{w}), 836(\mathrm{~m}) \mathrm{cm}^{-1}$. EI HRMS: Calcd for $\mathrm{C}_{17} \mathrm{H}_{28} \mathrm{O}_{4} \mathrm{Si}(\mathrm{M}+1)$ : 325.1835; Found: 325.1838 .

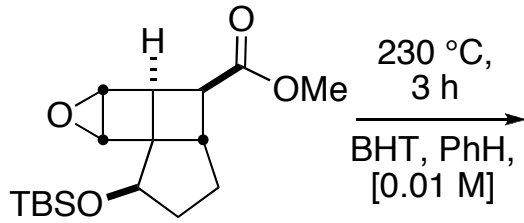

18

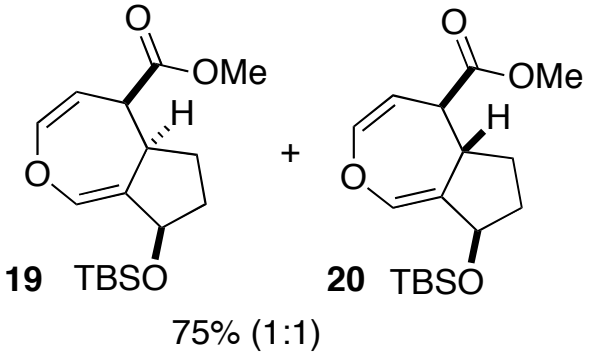


A degassed solution of $18(221 \mathrm{mg}, 0.683 \mathrm{mmol})$ and BHT (226 mg, $1.02 \mathrm{mmol})$ in benzene $(68.3 \mathrm{~mL})$ in a heavy-wall sealed tube $(350 \mathrm{~mL})$ was placed in a silicone oil bath and heated to $230{ }^{\circ} \mathrm{C}$. The reaction was monitored by TLC and stopped upon consumption of starting material $(3 \mathrm{~h})$. Concentration in vacuo, followed by filtration through basic alumina (1 cm plug) eluting with hexanes to remove the BHT afforded oxepines $\mathbf{1 9}$ and $\mathbf{2 0}$ as a slight yellow oil in $75 \%$ yield (166 mg, $0.512 \mathrm{mmol}, 1: 1 \mathrm{ratio}$ ).

Oxepines $19 \&$ 20: ${ }^{1} \mathrm{H}-\mathbf{N M R}\left(400 \mathrm{MHz}, \mathrm{CDCl}_{3}\right): \delta 6.39(\mathrm{~m}, 2 \mathrm{H}), 6.30(\mathrm{~d}, J=7.6 \mathrm{~Hz}, 1 \mathrm{H}), 6.22$ $(\mathrm{dd}, J=8.0,2.4 \mathrm{~Hz}, 1 \mathrm{H}), 4.75(\mathrm{t}, J=8.0 \mathrm{~Hz}, 1 \mathrm{H}), 4.69(\mathrm{dd}, J=7.6,2.4 \mathrm{~Hz}, 1 \mathrm{H}), 4.53(\mathrm{~m}, 2 \mathrm{H})$, $3.72(\mathrm{~s}, 3 \mathrm{H}), 3.66(\mathrm{~s}, 3 \mathrm{H}), 3.45(\mathrm{dd}, J=8.4,4.4 \mathrm{~Hz}, 1 \mathrm{H}), 3.20(\mathrm{~m}, 1 \mathrm{H}), 3.12(\mathrm{dt}, J=9.2,2.8 \mathrm{~Hz}$, $1 \mathrm{H}), 3.07(\mathrm{~m}, 1 \mathrm{H}), 1.68(\mathrm{~m}, 8 \mathrm{H}), 0.89(\mathrm{~d}, J=1.6 \mathrm{~Hz}, 18 \mathrm{H}), 0.07(\mathrm{dd}, J=4.0,2.0 \mathrm{~Hz}, 12 \mathrm{H})$.

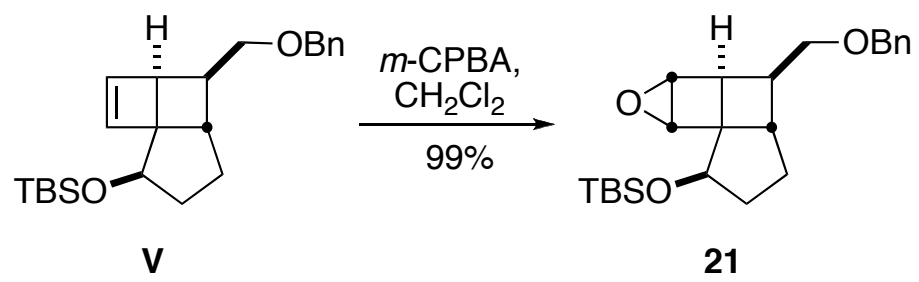

A small vial was charged with cyclobutene $\mathbf{V}(24 \mathrm{mg}, 0.066 \mathrm{mmol})$ in $\mathrm{CH}_{2} \mathrm{Cl}_{2}(0.2 \mathrm{~mL})$ and $m$-CPBA (19 mg, $0.11 \mathrm{mmol}$ ). The flask was flushed with $\mathrm{N}_{2}$ and allowed to stir at $\mathrm{rt}$. The consumption of starting material was monitored by TLC. Upon completion $(1.5 \mathrm{~h})$, the reaction was quenched with $\mathrm{Na}_{2} \mathrm{~S}_{2} \mathrm{O}_{3 \text { (sat. aq.) }}(1.0 \mathrm{~mL})$, diluted with $\mathrm{CH}_{2} \mathrm{Cl}_{2}(1.0 \mathrm{~mL})$ and allowed to stir for $1 \mathrm{~h}$. The mixture was diluted with $\mathrm{CH}_{2} \mathrm{Cl}_{2}(30 \mathrm{~mL})$ and the organic layer was separated, washed with $\mathrm{NaHCO}_{3 \text { (sat. aq.) }}$, dried over $\mathrm{MgSO}_{4}$, filtered and concentrated in vacuo. The resulting oil was purified by silica gel chromatography (2:1 hexane:Et $\left.{ }_{2} \mathrm{O}\right)$. Epoxide $\mathbf{2 1}$ was obtained in $99 \%$ yield as a colorless oil $(25 \mathrm{mg}, 0.65 \mathrm{mmol})$.

Epoxide 21: ${ }^{1} \mathbf{H}-\mathbf{N M R}\left(400 \mathrm{MHz}, \mathrm{CDCl}_{3}\right): \delta 7.34(\mathrm{~m}, 5 \mathrm{H}), 4.53(\mathrm{q}, J=12.4 \mathrm{~Hz}, 2 \mathrm{H}), 3.92(\mathrm{~m}$, $2 \mathrm{H}), 3.87(\mathrm{~d}, J=2.0 \mathrm{~Hz}, 1 \mathrm{H}), 3.57(\mathrm{~m}, 2 \mathrm{H}), 2.34(\mathrm{~m}, 3 \mathrm{H}), 2.02(\mathrm{~m}, 1 \mathrm{H}), 1.71(\mathrm{~m}, 2 \mathrm{H}), 1.52(\mathrm{~m}$, 1H), 0.88 (s, 9H), 0.07 (d, $J=14.4 \mathrm{~Hz}, 6 \mathrm{H}) .{ }^{13} \mathbf{C}-\mathbf{N M R}\left(100 \mathrm{MHz}, \mathrm{CDCl}_{3}\right): \delta 138.4,128.5$, $127.7,73.2,71.6,71.4,63.7,54.5,52.0,44.3,38.3,38.1,34.9,29.6,26.1,18.5,-4.5$. IR $(\mathrm{NaCl}$ thin film): 3048 (w), 2954 (s), 2885 (w), 2859 (m), 1471 (w), 1362 (w), 1255 (m), 1059 (m) cm ${ }^{-1}$. EI HRMS: Calcd for $\mathrm{C}_{23} \mathrm{H}_{34} \mathrm{O}_{3} \mathrm{Si}\left({ }^{+} \mathrm{M}\right)$ : 386.2277; Found: 386.2274 .
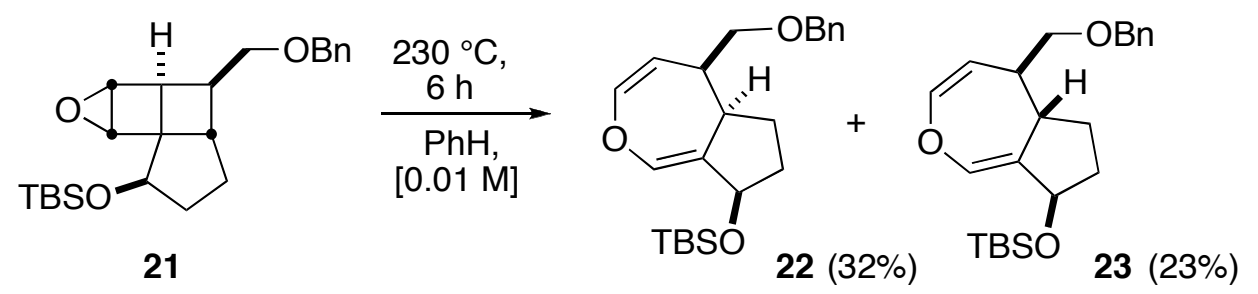

A degassed solution of $21(25.2 \mathrm{mg}, 0.0652 \mathrm{mmol})$ in benzene $(6.5 \mathrm{~mL})$ in a heavy-wall sealed tube $(35 \mathrm{~mL})$ was placed in a silicone oil bath and heated to $230{ }^{\circ} \mathrm{C}$. The reaction was monitored by TLC and stopped upon consumption of starting material ( $3 \mathrm{~h})$. Concentration in vacuo, followed by medium pressure liquid chromatography $\left(5 \% \mathrm{CH}_{2} \mathrm{Cl}_{2}\right.$ :hexane) with a basewashed column (1\% pyridine in EtOAc) was used to separate the diastereomers. Oxepine 22 was obtained as a colorless oil in $32 \%$ yield $(8.1 \mathrm{mg}, 0.021 \mathrm{mmol})$ and oxepine 23 was obtained as a colorless oil in $23 \%$ yield $(5.9 \mathrm{mg}, 0.015 \mathrm{mmol})$. 
Oxepine 22: ${ }^{1} \mathbf{H}-\mathrm{NMR}\left(400 \mathrm{MHz}, \mathrm{CDCl}_{3}\right): \delta 7.31(\mathrm{~m}, 5 \mathrm{H}), 6.35(\mathrm{t}, J=2.0 \mathrm{~Hz}, 1 \mathrm{H}), 6.17(\mathrm{dd}, J$ $=8.0,2.4 \mathrm{~Hz}, 1 \mathrm{H}), 4.73(\mathrm{dd}, J=8.0,2.4 \mathrm{~Hz}, 1 \mathrm{H}), 4.52(\mathrm{~m}, 3 \mathrm{H}), 3.49(\mathrm{dd}, J=9.2,3.6 \mathrm{~Hz}, 1 \mathrm{H})$, $3.31(\mathrm{dd}, J=9.2,7.2 \mathrm{~Hz}, 1 \mathrm{H}), 2.94(\mathrm{~m}, 1 \mathrm{H}), 2.41(\mathrm{~m}, 1 \mathrm{H}), 1.92(\mathrm{~m}, 2 \mathrm{H}), 1.54(\mathrm{~m}, 1 \mathrm{H}), 1.24(\mathrm{~m}$, 2H), 0.90 (s, 9H), 0.08 (d, $J=3.2 \mathrm{~Hz}, 6 \mathrm{H}) .{ }^{13}$ C-NMR (100 MHz, CD $\left.\mathrm{OD}\right): \delta$ 142.4, 140.0, $129.3,128.8,128.5,110.3,76.6,74.2,73.5,44.7,44.3,36.5,29.1,26.5,19.2,-4.0,-4.3$. IR (NaCl thin film): 3087 (w), 3037 (w), 2955 (s), 2860 (s), 1684 (w), 1653 (m), 1471 (w), 1361 $(\mathrm{m}), 1254(\mathrm{~m}), 1195(\mathrm{~m}), 1115(\mathrm{~s}), 866(\mathrm{~m}), 837(\mathrm{~m}), 426(\mathrm{~m}) \mathrm{cm}^{-1}$. EI HRMS: Calcd for $\mathrm{C}_{23} \mathrm{H}_{34} \mathrm{O}_{3} \mathrm{Si}\left({ }^{+} \mathrm{M}\right)$ : 386.2277; Found: 386.2277 .

Oxepine 23: ${ }^{1} \mathbf{H}-\mathbf{N M R}\left(400 \mathrm{MHz}, \mathrm{CDCl}_{3}\right): \delta 7.30(\mathrm{~m}, 5 \mathrm{H}), 6.34(\mathrm{~d}, J=2.4 \mathrm{~Hz}, 1 \mathrm{H}), 6.15(\mathrm{~d}, J=$ $8.0 \mathrm{~Hz}, 1 \mathrm{H}), 4.96(\mathrm{t}, J=8.0 \mathrm{~Hz}, 1 \mathrm{H}), 4.94(\mathrm{~m}, 3 \mathrm{H}), 3.74(\mathrm{dd}, J=8.8,5.2 \mathrm{~Hz}, 1 \mathrm{H}), 3.46(\mathrm{t}, J=8.0$ $\mathrm{Hz}, 1 \mathrm{H}), 3.10(\mathrm{~m}, 1 \mathrm{H}), 2.77(\mathrm{~m}, 1 \mathrm{H}), 1.90(\mathrm{~m}, 1 \mathrm{H}), 1.70(\mathrm{~m}, 2 \mathrm{H}), 1.55(\mathrm{~m}, 1 \mathrm{H}), 0.89(\mathrm{~s}, 9 \mathrm{H})$, $0.07(\mathrm{~d}, J=3.2 \mathrm{~Hz}, 6 \mathrm{H}) .{ }^{13} \mathrm{C}-\mathbf{N M R}\left(100 \mathrm{MHz} \mathrm{CDCl}_{3}\right): \delta 141.8,138.7,137.9,128.4,127.6$, $127.5,126.0,110.1,75.3,74.5,73.4,43.7,42.1,36.4,27.3,26.2,18.4,-4.1$. IR (NaCl thin film): 3060 (m), 3028 (s), 2954 (s), 2921 (s), 2859 (s), 1682 (m), 1649 (m), 1462 (w), 1331 (w), 1255 (m), $1183(\mathrm{~m}), 1129(\mathrm{~m}), 1092(\mathrm{~m}), 1037(\mathrm{~m}), 835(\mathrm{~m}) \mathrm{cm}^{-1}$. EI HRMS: Calcd for $\mathrm{C}_{23} \mathrm{H}_{34} \mathrm{O}_{3} \mathrm{Si}$ $\left({ }^{+} \mathrm{M}\right)$ : 386.2277; Found: 386.2280.

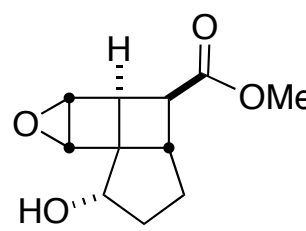

27

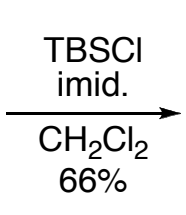

TBSO'"

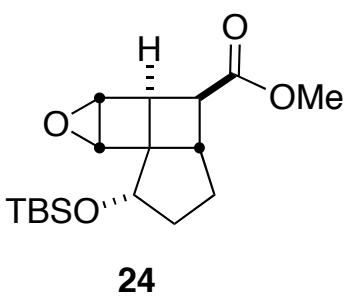

A round bottom flask $(10 \mathrm{~mL})$ was charged with epoxide $27(110 \mathrm{mg}, 0.523 \mathrm{mmol})$ in $\mathrm{CH}_{2} \mathrm{Cl}_{2}(1.0 \mathrm{~mL})$. $t$-Butyldimethylsilylchloride $(118 \mathrm{mg}, 0.785 \mathrm{mmol})$ and imidazole $(57.0 \mathrm{mg}$, $0.837 \mathrm{mmol}$ ) were added to the solution, which was then flushed with $\mathrm{N}_{2}$ and allowed to stir at rt. The consumption of starting material was monitored by TLC. Upon completion $(24 \mathrm{~h})$, the reaction was quenched with $\mathrm{NH}_{4} \mathrm{Cl}_{\text {(sat. aq.) }}(1.0 \mathrm{~mL})$ and diluted with hexanes $(4.0 \mathrm{~mL})$. The organic layer was separated, washed with $\mathrm{NaCl}_{\text {(sat. aq.) }}$, dried over $\mathrm{MgSO}_{4}$, filtered and concentrated in vacuo. The resulting oil was purified by silica gel chromatography (4:1 hexane:Et $\left.{ }_{2} \mathrm{O}\right)$. Epoxide 24 was obtained in $66 \%$ yield $(113 \mathrm{mg}, 0.348 \mathrm{mmol})$ as a colorless oil.

Epoxide 24: ${ }^{1} \mathrm{H}-\mathrm{NMR}\left(400 \mathrm{MHz}, \mathrm{CDCl}_{3}\right)$ : $\delta 4.07(\mathrm{~m}, 1 \mathrm{H}), 3.99(\mathrm{~d}, J=2.0 \mathrm{~Hz}, 1 \mathrm{H}), 3.82(\mathrm{dd}, J$ $=3.2,2.0 \mathrm{~Hz}, 1 \mathrm{H}), 3.71(\mathrm{~s}, 3 \mathrm{H}), 3.01(\mathrm{dd}, J=8.0,5.6 \mathrm{~Hz}, 1 \mathrm{H}), 2.91(\mathrm{dd}, J=8.0,3.2 \mathrm{~Hz}, 1 \mathrm{H})$, $2.73(\mathrm{~m}, 1 \mathrm{H}), 1.89(\mathrm{~m}, 1 \mathrm{H}), 1.69(\mathrm{~m}, 3 \mathrm{H}), 0.88(\mathrm{~s}, 9 \mathrm{H}), 0.08(\mathrm{~d}, J=4.8 \mathrm{~Hz}, 6 \mathrm{H}) .{ }^{13} \mathbf{C}-\mathbf{N M R}(100$ $\left.\mathrm{MHz}, \mathrm{CDCl}_{3}\right): \delta 173.3,73.6,61.2,54.8,51.9,51.7,42.2,41.9,39.5,35.2,28.0,26.2,18.7,-4.7$. IR (NaCl thin film): 2954 (s), 2886 (m), 2859 (m), 1737 (s), 1471 (w), 1437 (w), 1365 (w), 1255 $(\mathrm{m}), 1122(\mathrm{~m}), 865(\mathrm{~m}), 837(\mathrm{~m}), 779(\mathrm{w}), 431(\mathrm{~m}) \mathrm{cm}^{-1}$. EI HRMS: Calcd for $\mathrm{C}_{17} \mathrm{H}_{28} \mathrm{O}_{4} \mathrm{Si}$ $(\mathrm{M}+1)$ : 325.1835; Found: 325.1829. 


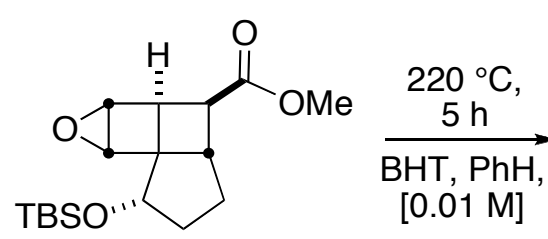

24

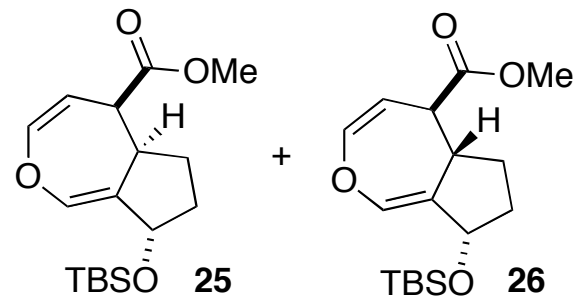

$72 \%(1: 3)$

A degassed solution of $24(32.5 \mathrm{mg}, 0.100 \mathrm{mmol})$ and BHT (44.0 mg, $0.200 \mathrm{mmol})$ in benzene $(10.0 \mathrm{~mL})$ in a heavy-wall sealed tube $(35 \mathrm{~mL})$ was placed in a silicone oil bath and heated to $220^{\circ} \mathrm{C}$. The reaction was monitored by TLC and stopped upon consumption of starting material $(5 \mathrm{~h})$. Concentration in vacuo, followed by filtration through basic alumina (1 cm plug) eluting with hexanes to remove the BHT afforded oxepines 25 and $\mathbf{2 6}$ as a slight yellow oil in $72 \%$ yield $(23.4 \mathrm{mg}, 0.0721 \mathrm{mmol}, 1: 3$ ratio).

Oxepines $25 \&$ 26: ${ }^{1} \mathrm{H}-\mathbf{N M R}\left(400 \mathrm{MHz}, \mathrm{CDCl}_{3}\right): \delta 6.40(\mathrm{~m}, 2 \mathrm{H}), 6.28(\mathrm{~d}, J=8.0 \mathrm{~Hz}, 1 \mathrm{H}), 6.22$ $(\mathrm{dd}, J=7.6,2.8 \mathrm{~Hz}, 1 \mathrm{H}), 4.77(\mathrm{~m}, 1 \mathrm{H}), 4.74(\mathrm{t}, J=8.0 \mathrm{~Hz}, 1 \mathrm{H}), 4.67(\mathrm{dd}, J=8.0,2.8 \mathrm{~Hz}, 1 \mathrm{H})$, $4.51(\mathrm{t}, J=4.4 \mathrm{~Hz}, 1 \mathrm{H}), 3.73(\mathrm{~s}, 3 \mathrm{H}), 3.67(\mathrm{~s}, 3 \mathrm{H}), 3.40(\mathrm{dd}, J=8.4,3.6 \mathrm{~Hz}, 1 \mathrm{H}), 3.26(\mathrm{dt}, J=$ 9.2, $2.8 \mathrm{~Hz}, 1 \mathrm{H}), 3.21(\mathrm{~m}, 1 \mathrm{H}), 3.02(\mathrm{~m}, 1 \mathrm{H}), 1.68(\mathrm{~m}, 8 \mathrm{H}), 0.90(\mathrm{~s}, 9 \mathrm{H}), 0.88(\mathrm{~d}, J=2.0 \mathrm{~Hz}$, $9 \mathrm{H}), 0.01(\mathrm{~d}, J=4.8 \mathrm{~Hz}, 6 \mathrm{H}), 0.07(\mathrm{~s}, 6 \mathrm{H})$.
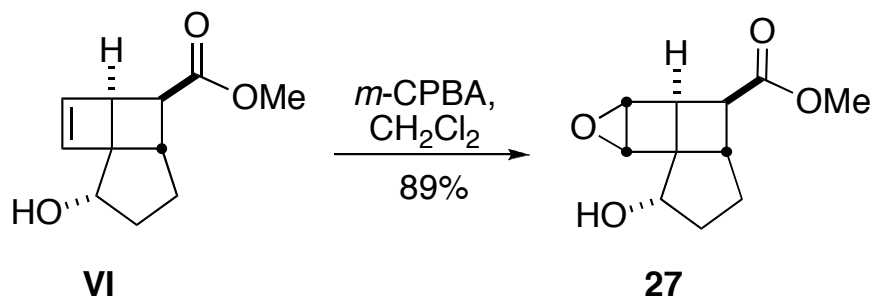

A round bottom flask (50 mL) was charged with cyclobutene VI (610 mg, $3.14 \mathrm{mmol})$ in $\mathrm{CH}_{2} \mathrm{Cl}_{2}(7.8 \mathrm{~mL})$ and $m$-CPBA $(596 \mathrm{mg}, 3.45 \mathrm{mmol})$. The flask was flushed with $\mathrm{N}_{2}$ and allowed to stir at rt. The consumption of starting material was monitored by TLC. Upon completion (1.5 h), the reaction was quenched with $\mathrm{Na}_{2} \mathrm{~S}_{2} \mathrm{O}_{3 \text { (sat. aq.) }}(5.0 \mathrm{~mL})$, diluted with $\mathrm{CH}_{2} \mathrm{Cl}_{2}(5.0 \mathrm{~mL})$ and allowed to stir for $1 \mathrm{~h}$. The mixture was diluted with $\mathrm{CH}_{2} \mathrm{Cl}_{2}(60 \mathrm{~mL})$ and the organic layer was separated, washed with $\mathrm{NaHCO}_{3 \text { (sat. aq.) }}$, dried over $\mathrm{MgSO}_{4}$, filtered and concentrated in vacuo. The resulting oil was purified by silica gel chromatography (1:2 hexane:Et $\left.{ }_{2} \mathrm{O}\right)$. Epoxide 27 was obtained in $89 \%$ yield as a white solid $(577 \mathrm{mg}, 2.74 \mathrm{mmol})$.

Epoxide 27: $\mathbf{m p}=84-86{ }^{\circ} \mathrm{C} .{ }^{1} \mathbf{H}-\mathrm{NMR}\left(400 \mathrm{MHz}, \mathrm{CDCl}_{3}\right): \delta 4.16(\mathrm{dd}, J=9.6,6.4 \mathrm{~Hz}, 1 \mathrm{H})$, $4.09(\mathrm{~d}, J=2.0 \mathrm{~Hz}, 1 \mathrm{H}), 4.01(\mathrm{t}, J=3.2 \mathrm{~Hz}, 1 \mathrm{H}), 3.73(\mathrm{~s}, 3 \mathrm{H}), 3.02(\mathrm{~m}, 2 \mathrm{H}), 2.80(\mathrm{dd}, J=8.0$, $4.4 \mathrm{~Hz}, 1 \mathrm{H}), 2.10(\mathrm{~m}, 1 \mathrm{H}), 1.85(\mathrm{~m}, 2 \mathrm{H}), 1.69(\mathrm{~m}, 2 \mathrm{H}) .{ }^{13} \mathbf{C}-\mathbf{N M R}\left(100 \mathrm{MHz}, \mathrm{CDCl}_{3}\right): \delta 172.9$, 73.3, 61.7, 55.4, 51.9, 51.8, 41.8, 41.7, 38.8, 33.6, 28.1. IR (KBr pellet): 3466 (br), 2987 (m), 2960 (m), 2867 (m), 2355 (w), 2333 (w), 1723 (s), 1435 (m), 1310 (m), 1217 (s), 1085 (s), 813 (s) $\mathrm{cm}^{-1}$. HRMS (m/z): Calcd for $(\mathrm{M}+\mathrm{Na})$ : 233.0790. Found: 233.0792. 


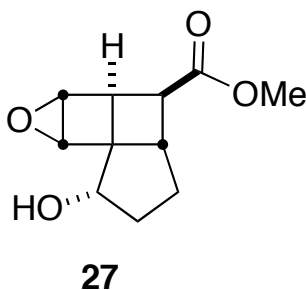

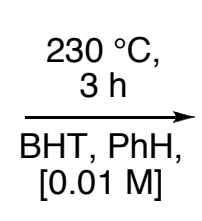

27

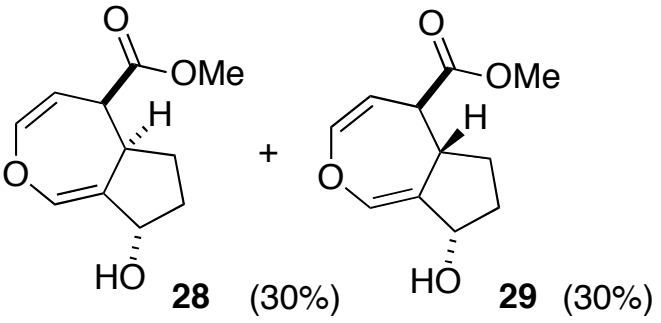

A degassed solution of 27 (100 mg, $0.476 \mathrm{mmol})$ and BHT (157 mg, $0.714 \mathrm{mmol})$ in benzene $(47.6 \mathrm{~mL})$ in a heavy-wall sealed tube $(350 \mathrm{~mL})$ was placed in a silicone oil bath and heated to $230{ }^{\circ} \mathrm{C}$. The reaction was monitored by TLC and stopped upon consumption of starting material $(3 \mathrm{~h})$. Concentration in vacuo, followed by filtration through basic alumina (1 cm plug) with hexanes (to separate BHT) followed by $\mathrm{Et}_{2} \mathrm{O}$ afforded two diastereomers as a colorless oil. Medium pressure liquid chromatography $\left(1 \% \mathrm{MeOH}: \mathrm{CH}_{2} \mathrm{Cl}_{2}\right)$ with a base-washed column (1\% pyridine in EtOAc) was used to separate the diastereomers. Oxepine $\mathbf{2 8}$ was obtained as a light yellow oil in $30 \%$ yield (30 mg, $0.14 \mathrm{mmol}$ ) and oxepine 29 was obtained as a light yellow oil in $30 \%$ yield $(30 \mathrm{mg}, 0.14 \mathrm{mmol})$.

Oxepine 28: ${ }^{1} \mathbf{H}-\mathbf{N M R}\left(400 \mathrm{MHz}, \mathrm{CDCl}_{3}\right): \delta 6.58(\mathrm{~s}, 1 \mathrm{H}), 6.30(\mathrm{~d}, J=8.0 \mathrm{~Hz}, 1 \mathrm{H}), 4.80(\mathrm{t}, J=$ $8.0 \mathrm{~Hz}, 1 \mathrm{H}), 4.76(\mathrm{~m}, 1 \mathrm{H}), 3.73(\mathrm{~s}, 1 \mathrm{H}), 3.66(\mathrm{~s}, 3 \mathrm{H}), 3.45(\mathrm{dd}, J=8.4,4.0 \mathrm{~Hz}, 1 \mathrm{H}), 3.23(\mathrm{~m}$, 1H), $2.10(\mathrm{~m}, 1 \mathrm{H}), 1.94(\mathrm{~m}, 1 \mathrm{H}), 1.58(\mathrm{~m}, 2 \mathrm{H}) .{ }^{13} \mathbf{C}-\mathbf{N M R}\left(100 \mathrm{MHz}, \mathrm{CDCl}_{3}\right): \delta$ 172.3, 144.0, 138.1, 126.8, 103.2, 75.9, 52.1, 48.2, 42.5, 35.5, 28.5. IR ( NaCl thin film): 3417 (br), 2925 (s), 2853 (m), 1733 (s), 1683 (s), 1652 (s), 1436 (m), 1313 (m), 1197 (s), 1093 (m), 1037 (w), 993 (w), $961(\mathrm{w}), 855(\mathrm{w}), 736(\mathrm{~m}), 701(\mathrm{w}) \mathrm{cm}^{-1}$. EI HRMS: Calcd for $\mathrm{C}_{11} \mathrm{H}_{14} \mathrm{O}_{4}\left({ }^{+} \mathrm{M}\right)$ : 210.0892; Found: 210.0890.

Oxepine 29: ${ }^{1} \mathbf{H}-\mathbf{N M R}\left(400 \mathrm{MHz}, \mathrm{CDCl}_{3}\right): \delta 6.58(\mathrm{~d}, J=2.4 \mathrm{~Hz}, 1 \mathrm{H}), 6.22(\mathrm{dd}, J=8.0,2.8 \mathrm{~Hz}$, $1 \mathrm{H}), 4.71(\mathrm{dd}, J=8.0,2.4 \mathrm{~Hz}, 1 \mathrm{H}), 4.57(\mathrm{~m}, 1 \mathrm{H}), 3.73(\mathrm{~s}, 3 \mathrm{H}), 3.28(\mathrm{dt}, J=8.8,2.4 \mathrm{~Hz}, 1 \mathrm{H})$, $3.07(\mathrm{~m}, 1 \mathrm{H}), 1.85(\mathrm{~m}, 5 \mathrm{H}) .{ }^{13} \mathbf{C}-\mathbf{N M R}\left(100 \mathrm{MHz}, \mathrm{CDCl}_{3}\right): \delta 173.2,142.3,140.3,127.8,105.1$, 75.1, 52.3, 49.6, 45.2, 35.5, 29.5. IR (NaCl thin film): 3430 (br), 2954 (m), 1736 (s), 1681 (m), $1654(\mathrm{~m}), 1436(\mathrm{~m}), 1352(\mathrm{~m}), 1268(\mathrm{~m}), 1196(\mathrm{~m}), 1171(\mathrm{~m}), 1024(\mathrm{~m}), 737(\mathrm{~s}), 703(\mathrm{~m}) . \mathbf{E I}$ HRMS: Calcd for $\mathrm{C}_{11} \mathrm{H}_{14} \mathrm{O}_{4}\left({ }^{+} \mathrm{M}\right)$ : 210.0892; Found: 210.0897.

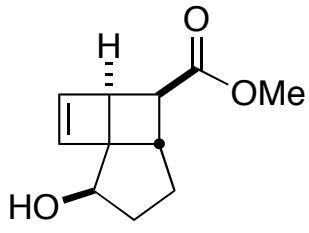

VII
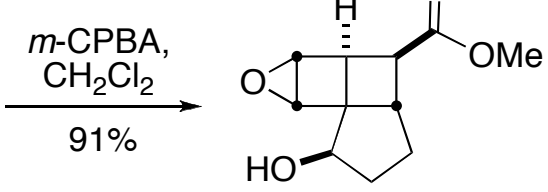

30

A round bottom flask ( $5 \mathrm{~mL}$ ) was charged with cyclobutene VII ( $83 \mathrm{mg}, 0.43 \mathrm{mmol})$ in $\mathrm{CH}_{2} \mathrm{Cl}_{2}(1.2 \mathrm{~mL})$ and $m$-CPBA $(81 \mathrm{mg}, 0.47 \mathrm{mmol})$. The flask was flushed with $\mathrm{N}_{2}$ and allowed to stir at rt. The consumption of starting material was monitored by TLC. Upon completion (1.5 h) the reaction was quenched with $\mathrm{Na}_{2} \mathrm{~S}_{2} \mathrm{O}_{3 \text { (sat. aq.) }}(1.0 \mathrm{~mL})$, diluted with $\mathrm{CH}_{2} \mathrm{Cl}_{2}(1.0 \mathrm{~mL})$ and allowed to stir for $1 \mathrm{~h}$. The mixture was diluted with $\mathrm{CH}_{2} \mathrm{Cl}_{2}(30 \mathrm{~mL})$ and the organic layer was separated, washed with $\mathrm{NaHCO}_{3 \text { (sat. aq.) }}$, dried over $\mathrm{MgSO}_{4}$, filtered and concentrated in vacuo. The resulting oil was purified by silica gel chromatography (1:1 hexane:Et $\left.\mathrm{t}_{2} \mathrm{O}\right)$. Epoxide $\mathbf{3 0}$ was obtained in $91 \%$ yield as a white solid $(82 \mathrm{mg}, 0.39 \mathrm{mmol})$. 
Epoxide 30: $\mathbf{m p}=53-56{ }^{\circ} \mathrm{C} .{ }^{1} \mathbf{H}-\mathbf{N M R}\left(400 \mathrm{MHz}, \mathrm{CDCl}_{3}\right): \delta 4.10(\mathrm{~d}, J=2.0 \mathrm{~Hz}, 1 \mathrm{H}), 4.04(\mathrm{~m}$, 2H), $3.73(\mathrm{~s}, 3 \mathrm{H}), 2.93(\mathrm{~m}, 2 \mathrm{H}), 2.57(\mathrm{dd}, J=8.0,4.0 \mathrm{~Hz}, 1 \mathrm{H}), 2.14(\mathrm{~m}, 1 \mathrm{H}), 1.91(\mathrm{dd}, J=13.2$, $6.8 \mathrm{~Hz}, 1 \mathrm{H}), 1.81(\mathrm{~m}, 2 \mathrm{H}), 1.68(\mathrm{dd}, J=13.2,7.2 \mathrm{~Hz}, 1 \mathrm{H}) .{ }^{13} \mathbf{C}-\mathbf{N M R}\left(100 \mathrm{MHz}, \mathrm{CDCl}_{3}\right): \delta$ 173.2, 71.0, 63.6, 53.4, 52.2, 52.0, 44.3, 41.2, 39.4, 34.1, 29.5. IR (KBr pellet): 3455 (br), 2971 (m), 2905 (m), 1718 (s), 1440 (m), 1212 (s), 819 (m) $\mathrm{cm}^{-1}$. HRMS (m/z): Calcd for (M + Na): 233.0790. Found: 233.0783.

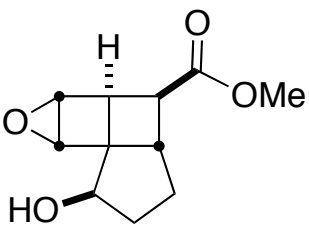

30

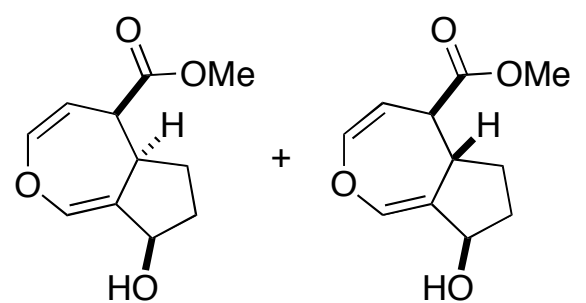

$31(38 \%)$

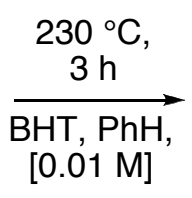

$[0.01 \mathrm{M}]$

A degassed solution of 30 (24 mg, $0.11 \mathrm{mmol})$ and BHT (38 $\mathrm{mg}, 0.17 \mathrm{mmol})$ in benzene $(11.4 \mathrm{~mL})$ in a heavy-wall sealed tube $(35 \mathrm{~mL})$ was placed in a silicone oil bath and heated to $230{ }^{\circ} \mathrm{C}$. The reaction was monitored by TLC and stopped upon consumption of starting material $(3 \mathrm{~h})$. Concentration in vacuo, followed by filtration through basic alumina $(1 \mathrm{~cm}$ plug $)$ with hexanes (to separate $\mathrm{BHT}$ ) followed by $\mathrm{Et}_{2} \mathrm{O}$ afforded two diastereomers as a colorless oil. Medium pressure liquid chromatography (10-30\% hexane:Et $\left.{ }_{2} \mathrm{O}\right)$ with a base-washed column (1\% pyridine in EtOAc) was used to separate the diastereomers. Oxepine $\mathbf{3 1}$ was obtained as a colorless oil in 38\% yield $(9.0 \mathrm{mg}, 0.043 \mathrm{mmol})$ and oxepine 32 was obtained as a colorless oil in $31 \%$ yield $(7.5 \mathrm{mg}, 0.036 \mathrm{mmol})$.

Oxepine 31: ${ }^{1} \mathbf{H}-\mathbf{N M R}\left(400 \mathrm{MHz}, \mathrm{CDCl}_{3}\right): \delta 6.66(\mathrm{~d}, J=2.8 \mathrm{~Hz}, 1 \mathrm{H}), 6.27(\mathrm{~d}, J=8.0 \mathrm{~Hz}, 1 \mathrm{H})$, $4.79(\mathrm{t}, J=8.0 \mathrm{~Hz}, 1 \mathrm{H}), 4.50(\mathrm{~m}, 1 \mathrm{H}), 3.79(\mathrm{~d}, J=8.0 \mathrm{~Hz}, 1 \mathrm{H}), 3.72(\mathrm{~s}, 3 \mathrm{H}), 3.52(\mathrm{dd}, J=8.0$, $2.8 \mathrm{~Hz}, 1 \mathrm{H}), 3.02(\mathrm{~m}, 1 \mathrm{H}), 1.80(\mathrm{~m}, 4 \mathrm{H}) .{ }^{13} \mathbf{C}-\mathbf{N M R}\left(100 \mathrm{MHz}, \mathrm{CDCl}_{3}\right): \delta 173.5,144.0,139.4$, 128.2, 102.2, 75.1 , 52.5, 47.0, 43.3, 37.4, 28.8. IR (NaCl thin film): 3497 (br), 2955 (s), 2878 (w), 1725 (s), 1649 (m), 1436 (w), 1309 (w), 1201 (s) cm $\mathrm{cm}^{-1}$. EI HRMS: Calcd for $\mathrm{C}_{11} \mathrm{H}_{14} \mathrm{O}_{4}$ $\left({ }^{+} \mathrm{M}\right)$ : 210.0892; Found: 210.0897.

Oxepine 32: ${ }^{1} \mathbf{H}-\mathrm{NMR}\left(400 \mathrm{MHz}, \mathrm{CDCl}_{3}\right): \delta 6.55(\mathrm{~s}, 1 \mathrm{H}), 6.23(\mathrm{dd}, J=8.0 \mathrm{~Hz}, 2.4 \mathrm{~Hz}, 1 \mathrm{H})$, $4.77(\mathrm{dd}, J=8.0,2.4 \mathrm{~Hz}, 1 \mathrm{H}), 4.59(\mathrm{t}, J=5.6 \mathrm{~Hz}, 1 \mathrm{H}), 3.73(\mathrm{~s}, 3 \mathrm{H}), 3.22(\mathrm{~m}, 1 \mathrm{H}), 3.14(\mathrm{dt}, J=$ 9.2, $2.8 \mathrm{~Hz}, 1 \mathrm{H}), 2.10(\mathrm{~m}, 1 \mathrm{H}), 1.94(\mathrm{~m}, 1 \mathrm{H}), 1.55(\mathrm{~m}, 3 \mathrm{H}) .{ }^{13} \mathbf{C}-\mathbf{N M R}\left(100 \mathrm{MHz}, \mathrm{CDCl}_{3}\right): \delta$ 173.2, 142.3, 139.8, 127.0, 106.0, 75.6, 52.3, 48.9, 44.0, 34.6, 29.3. IR (NaCl thin film): 3423 (br), 2954 (m), 1736 (s), 1681 (m), 1655 (m), 1437 (m), 1350 (m), 1274 (m), 1193 (m), 1167 (m), $1134(\mathrm{~m}), 1059(\mathrm{~m}), 1028(\mathrm{~m}), 739(\mathrm{~m}) \mathrm{cm}^{-1}$. EI HRMS: Calcd for $\mathrm{C}_{11} \mathrm{H}_{14} \mathrm{O}_{4}\left({ }^{+} \mathrm{M}\right)$ : 210.0892; Found: 210.0888.

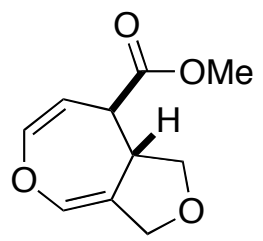

8
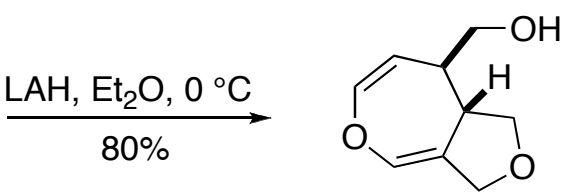

VIII 
A round bottom flask $(5 \mathrm{~mL})$ was charged with oxepine $8(30.8 \mathrm{mg}, 0.157 \mathrm{mmol})$ in $\mathrm{Et}_{2} \mathrm{O}$ $(2.0 \mathrm{~mL})$. The solution was then flushed with $\mathrm{N}_{2}$ and cooled to $0^{\circ} \mathrm{C}$ in an ice bath. An LAH solution $\left(0.47 \mathrm{~mL}, 0.47 \mathrm{mmol}, 1 \mathrm{M}\right.$ in $\left.\mathrm{Et}_{2} \mathrm{O}\right)$ was added dropwise by syringe to the reaction. The consumption of starting material was monitored by TLC. Upon completion $(1 \mathrm{~h})$, the reaction was treated with of $\mathrm{H}_{2} \mathrm{O}(50 \mu \mathrm{L}), 15 \%$ aqueous $\mathrm{NaOH}(50 \mu \mathrm{L})$, and then $\mathrm{H}_{2} \mathrm{O}(150 \mu \mathrm{L})$. After this treatment, a white precipitate formed and settled to the bottom of the flask. The reaction mixture was filtered and the salts were washed with $\mathrm{Et}_{2} \mathrm{O}(3 \times 5 \mathrm{~mL})$. The filtrate was concentrated in vacuo to afford a colorless oil. The resulting oil was purified by medium pressure liquid chromatography (1.5:1 hexane:EtOAc) using a base-washed column (1\% pyridine in EtOAc). Oxepine VIII was obtained in $80 \%$ yield $(21 \mathrm{mg}, 0.12 \mathrm{mmol})$ as a colorless oil.

Oxepine VIII: ${ }^{1} \mathbf{H}-\mathbf{N M R}\left(400 \mathrm{MHz}, \mathrm{CDCl}_{3}\right): \delta 6.36(\mathrm{~d}, J=2.4 \mathrm{~Hz}, 1 \mathrm{H}), 6.25(\mathrm{dd}, J=7.6,2.4$ $\mathrm{Hz}, 1 \mathrm{H}), 4.64(\mathrm{dd}, J=8.0,2.8 \mathrm{~Hz}, 1 \mathrm{H}), 4.38(\mathrm{~d}, J=11.6 \mathrm{~Hz}, 1 \mathrm{H}), 4.28(\mathrm{dt}, J=12.0,2.0 \mathrm{~Hz}, 1 \mathrm{H})$, $4.20(\mathrm{t}, J=8.0 \mathrm{~Hz}, 1 \mathrm{H}), 3.59(\mathrm{~m}, 4 \mathrm{H}), 3.09(\mathrm{q}, J=8.0 \mathrm{~Hz}, 1 \mathrm{H}), 2.54(\mathrm{~m}, 1 \mathrm{H}) .{ }^{13} \mathrm{C}-\mathbf{N M R}(100$ $\left.\mathrm{MHz}, \mathrm{CDCl}_{3}\right): \delta 142.7,134.5,121.2,107.5,73.5,70.1,65.9,44.8,43.1$. IR (NaCl thin film): 3421 (br), 2921 (s), 2856 (s), 1694 (s), 1654 (s), 1359 (m), 1308 (m), 1206 (m), 1160 (s), 1108 (m), 1071 (m), 1039 (s), 967 (m), 927 (m), 867 (m), 747 (m), 709 (m) cm $\mathrm{cm}^{-1}$ EI HRMS: Calcd for $\mathrm{C}_{9} \mathrm{H}_{12} \mathrm{O}_{3}(+\mathrm{M})$ : 168.0787; Found: 168.0786.

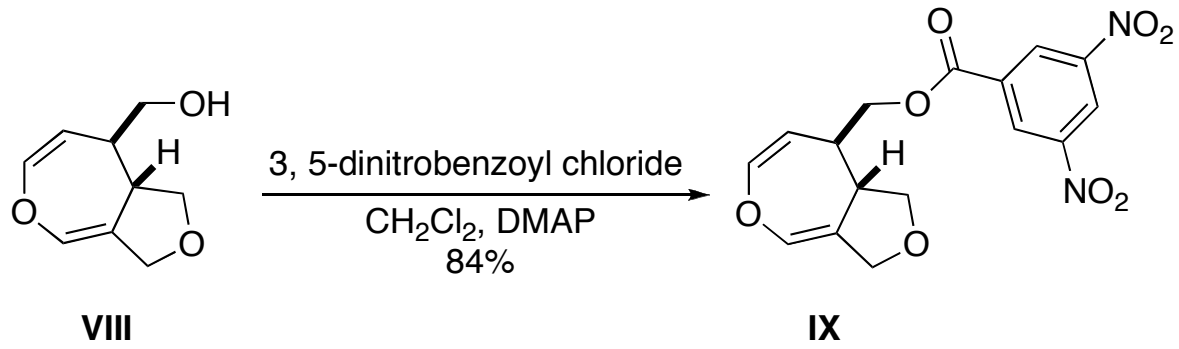

A round bottom flask $(10 \mathrm{~mL})$ was charged with oxepine VIII $(10.0 \mathrm{mg}, 0.0595 \mathrm{mmol})$ in $\mathrm{CH}_{2} \mathrm{Cl}_{2}$ (2.0 mL). 3,5-Dinitrobenzoyl chloride (20.6 mg, $0.0892 \mathrm{mmol}$ ) was added to the reaction vessel as a solid. The reaction mixture was then flushed with $\mathrm{N}_{2}$ and cooled to $0{ }^{\circ} \mathrm{C}$ in an ice bath. 4-Dimethylaminopyridine $(10.9 \mathrm{mg}, 0.0892 \mathrm{mmol})$ dissolved $\mathrm{CH}_{2} \mathrm{Cl}_{2}(1.0 \mathrm{~mL})$ was added to the reaction by syringe. The consumption of starting material was monitored by TLC. Upon completion $\left(1.5 \mathrm{~h}\right.$ ), the reaction was quenched with $\mathrm{NH}_{4} \mathrm{Cl}_{\text {(sat. aq.) }}(2.0 \mathrm{~mL})$. The organic layer was separated and the aqueous layer was extracted with $\mathrm{CH}_{2} \mathrm{Cl}_{2}(4 \times 4.0 \mathrm{~mL})$. The organic layers were combined, dried over $\mathrm{MgSO}_{4}$, filtered and concentrated in vacuo. The resulting solid was purified by medium pressure liquid chromatography (3:1 hexane:EtOAc) using a basewashed column (1\% pyridine in EtOAc). Oxepine IX was obtained in $84 \%$ yield $(18.1 \mathrm{mg}$, $0.0500 \mathrm{mmol}$ ) as a thick yellow gum and was crystallized by slow evaporation of $t$-butyl methyl ether to afford yellow crystals suitable for X-ray analysis.

Oxepine IX: $\mathbf{m p}=121-122{ }^{\circ} \mathrm{C} .{ }^{1} \mathbf{H}-\mathbf{N M R}\left(400 \mathrm{MHz}, \mathrm{CDCl}_{3}\right): \delta 9.25(\mathrm{t}, J=2.8 \mathrm{~Hz}, 1 \mathrm{H}), 9.14$ $(\mathrm{d}, J=2.8 \mathrm{~Hz}, 2 \mathrm{H}), 6.40(\mathrm{~d}, J=2.8 \mathrm{~Hz}, 1 \mathrm{H}), 6.27(\mathrm{dd}, J=10.8,3.6 \mathrm{~Hz}, 1 \mathrm{H}), 4.69$ (dd, $J=10.8$, $3.6 \mathrm{~Hz}, 1 \mathrm{H}), 4.38(\mathrm{~m}, 4 \mathrm{H}), 4.23(\mathrm{t}, J=10.8 \mathrm{~Hz}, 1 \mathrm{H}), 3.66(\mathrm{t}, J=11.6 \mathrm{~Hz}, 1 \mathrm{H}), 3.12(\mathrm{~m}, 1 \mathrm{H}), 2.91$ $(\mathrm{m}, 1 \mathrm{H}) .{ }^{13} \mathbf{C}-\mathbf{N M R}\left(100 \mathrm{MHz}, \mathrm{CDCl}_{3}\right): \delta 162.5,148.8,142.9,134.9,133.5,129.5,122.7,120.5$, 106.1, 73.2, 70.2, 69.1, 45.1, 40.1. IR (NaCl thin film): $3099(\mathrm{~m}), 2919(\mathrm{~m}), 2850(\mathrm{~m}), 2360$ (w), 2342 (w), 1732 (s), 1695 (m), 1655 (m), 1629 (m), 1597 (w), 1544 (s), 1462 (m), 1345 (s), 
1278 (s), 1207 (w), 1162 (s), 1120 (m), 1075 (m), 1027 (m), 974 (w), 923 (m), 867 (w), 831 (w), 773 (w), 730 (s), 721 (s) $\mathrm{cm}^{-1}$. EI HRMS: Calcd for $\mathrm{C}_{16} \mathrm{H}_{14} \mathrm{~N}_{2} \mathrm{O}_{8}\left({ }^{+} \mathrm{M}\right)$ : 362.0750; Found: 362.0746 .

\section{X-ray Crystal Structure of Compound 6}

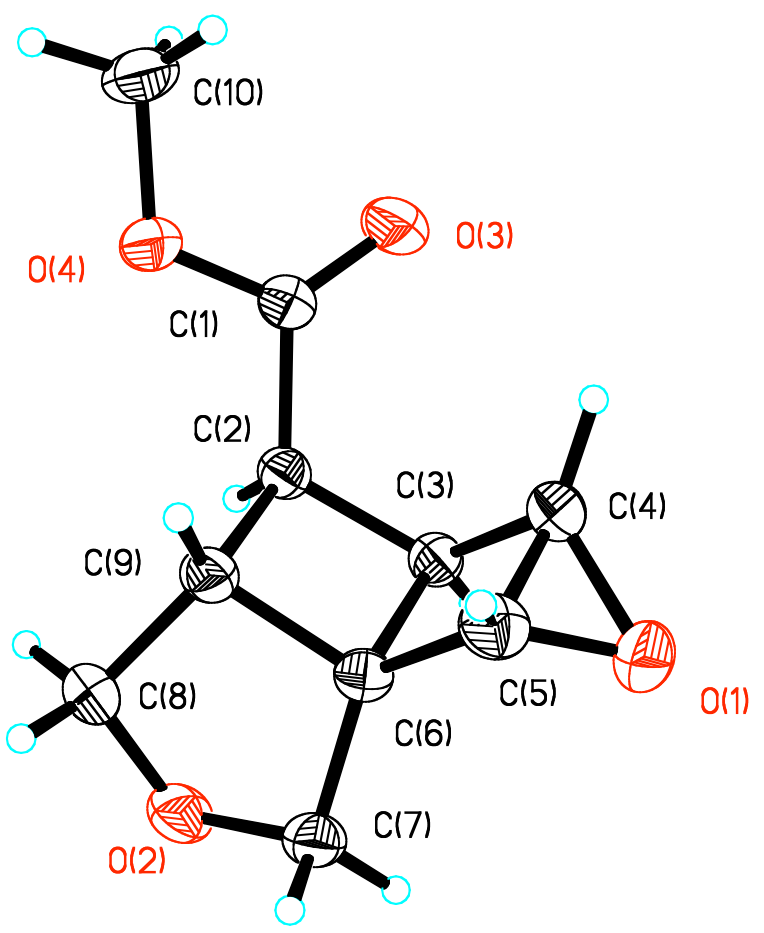

Table 1. Crystal data and structure refinement for Compound 6.

Identification code

Empirical formula

Formula weight

Temperature

Wavelength

Crystal system

Space group

Unit cell dimensions

Volume

Z

Density (calculated)

Absorption coefficient

$\mathrm{F}(000)$

Crystal size

Theta range for data collection

Index ranges

Reflections collected

Independent reflections aj101t

C10 H12 O4

196.20

193(2) K

$0.71073 \AA$

Monoclinic

$\mathrm{P} 2(1) / \mathrm{c}$

$\mathrm{a}=6.0680(6) \AA$

$\mathrm{b}=10.6903(10) \AA$

$\mathrm{c}=14.2193(14) \AA$

921.41(15) $\AA^{3}$

4

$1.414 \mathrm{Mg} / \mathrm{m}^{3}$

$0.110 \mathrm{~mm}^{-1}$

416

$0.60 \times 0.10 \times 0.10 \mathrm{~mm}^{3}$

2.38 to $28.28^{\circ}$.

$-6<=\mathrm{h}<=8,-14<=\mathrm{k}<=13,-18<=\mathrm{l}<=15$

6682

$2283[\mathrm{R}($ int $)=0.0504]$ 
Completeness to theta $=28.28^{\circ}$

Absorption correction

Refinement method

Data / restraints / parameters

Goodness-of-fit on $\mathrm{F}^{2}$

Final $\mathrm{R}$ indices [I $>2 \operatorname{sigma}(\mathrm{I})]$

$\mathrm{R}$ indices (all data)

Largest diff. peak and hole
$99.8 \%$

None

Full-matrix least-squares on $\mathrm{F}^{2}$

2283 / 0 / 127

1.004

$\mathrm{R} 1=0.0472, \mathrm{wR} 2=0.1265$

$\mathrm{R} 1=0.0578, \mathrm{wR} 2=0.1318$

0.415 and -0.315 e. $\AA^{-3}$

Table 2. Atomic coordinates $\left(\mathrm{x} 10^{4}\right)$ and equivalent isotropic displacement parameters $\left(\AA^{2} \times 10^{3}\right)$ For Compound 6. U(eq) is defined as one third of the trace of the orthogonalized $U^{i j}$ tensor.

\begin{tabular}{lrrrr}
\hline & $\mathrm{x}$ & $\mathrm{y}$ & $\mathrm{z}$ & $\mathrm{U}(\mathrm{eq})$ \\
\hline $\mathrm{O}(2)$ & $2330(2)$ & $4744(1)$ & $4074(1)$ & $35(1)$ \\
$\mathrm{O}(1)$ & $5821(2)$ & $8076(1)$ & $3035(1)$ & $39(1)$ \\
$\mathrm{O}(4)$ & $-1515(2)$ & $6142(1)$ & $1082(1)$ & $35(1)$ \\
$\mathrm{C}(7)$ & $3525(2)$ & $5889(1)$ & $4229(1)$ & $29(1)$ \\
$\mathrm{C}(3)$ & $3964(2)$ & $6327(1)$ & $2361(1)$ & $24(1)$ \\
$\mathrm{C}(6)$ & $2993(2)$ & $6633(1)$ & $3351(1)$ & $23(1)$ \\
$\mathrm{C}(9)$ & $689(2)$ & $6175(1)$ & $2977(1)$ & $27(1)$ \\
$\mathrm{C}(2)$ & $1693(2)$ & $5810(1)$ & $2033(1)$ & $23(1)$ \\
$\mathrm{O}(3)$ & $1657(2)$ & $6729(1)$ & $495(1)$ & $52(1)$ \\
$\mathrm{C}(1)$ & $654(2)$ & $6294(1)$ & $1124(1)$ & $26(1)$ \\
$\mathrm{C}(5)$ & $3499(2)$ & $7994(1)$ & $3196(1)$ & $32(1)$ \\
$\mathrm{C}(4)$ & $4327(2)$ & $7728(1)$ & $2258(1)$ & $30(1)$ \\
$\mathrm{C}(8)$ & $236(3)$ & $5102(2)$ & $3647(1)$ & $38(1)$ \\
$\mathrm{C}(10)$ & $-2670(3)$ & $6565(2)$ & $225(1)$ & $39(1)$ \\
& & & & \\
\hline
\end{tabular}

Table 3. Bond lengths $[\AA]$ and angles $\left[^{\circ}\right]$ for Compound 6.

\begin{tabular}{lc}
\hline $\mathrm{O}(2)-\mathrm{C}(7)$ & $1.4344(17)$ \\
$\mathrm{O}(2)-\mathrm{C}(8)$ & $1.4347(18)$ \\
$\mathrm{O}(1)-\mathrm{C}(5)$ & $1.4400(19)$ \\
$\mathrm{O}(1)-\mathrm{C}(4)$ & $1.4442(16)$ \\
$\mathrm{O}(4)-\mathrm{C}(1)$ & $1.3248(16)$ \\
$\mathrm{O}(4)-\mathrm{C}(10)$ & $1.4494(15)$ \\
$\mathrm{C}(7)-\mathrm{C}(6)$ & $1.5024(17)$ \\
$\mathrm{C}(3)-\mathrm{C}(4)$ & $1.5219(18)$ \\
$\mathrm{C}(3)-\mathrm{C}(2)$ & $1.5372(17)$ \\
$\mathrm{C}(3)-\mathrm{C}(6)$ & $1.5849(18)$ \\
$\mathrm{C}(6)-\mathrm{C}(5)$ & $1.5055(18)$ \\
$\mathrm{C}(6)-\mathrm{C}(9)$ & $1.5519(17)$ \\
$\mathrm{C}(9)-\mathrm{C}(8)$ & $1.525(2)$ \\
$\mathrm{C}(9)-\mathrm{C}(2)$ & $1.5490(18)$ \\
$\mathrm{C}(2)-\mathrm{C}(1)$ & $1.5041(17)$ \\
$\mathrm{O}(3)-\mathrm{C}(1)$ & $1.1987(17)$ \\
$\mathrm{C}(5)-\mathrm{C}(4)$ & $1.474(2)$ \\
$\mathrm{C}(7)-\mathrm{O}(2)-\mathrm{C}(8)$ & \\
$\mathrm{C}(5)-\mathrm{O}(1)-\mathrm{C}(4)$ & $105.61(10)$ \\
$\mathrm{C}(1)-\mathrm{O}(4)-\mathrm{C}(10)$ & $11.47(9)$ \\
$\mathrm{O}(2)-\mathrm{C}(7)-\mathrm{C}(6)$ & $103.69(10)$ \\
&
\end{tabular}




$\begin{array}{lc}\mathrm{C}(4)-\mathrm{C}(3)-\mathrm{C}(2) & 117.17(10) \\ \mathrm{C}(4)-\mathrm{C}(3)-\mathrm{C}(6) & 86.78(10) \\ \mathrm{C}(2)-\mathrm{C}(3)-\mathrm{C}(6) & 88.82(9) \\ \mathrm{C}(7)-\mathrm{C}(6)-\mathrm{C}(5) & 126.60(11) \\ \mathrm{C}(7)-\mathrm{C}(6)-\mathrm{C}(9) & 105.87(10) \\ \mathrm{C}(5)-\mathrm{C}(6)-\mathrm{C}(9) & 116.18(11) \\ \mathrm{C}(7)-\mathrm{C}(6)-\mathrm{C}(3) & 123.63(11) \\ \mathrm{C}(5)-\mathrm{C}(6)-\mathrm{C}(3) & 89.00(10) \\ \mathrm{C}(9)-\mathrm{C}(6)-\mathrm{C}(3) & 89.76(9) \\ \mathrm{C}(8)-\mathrm{C}(9)-\mathrm{C}(2) & 116.16(12) \\ \mathrm{C}(8)-\mathrm{C}(9)-\mathrm{C}(6) & 102.04(10) \\ \mathrm{C}(2)-\mathrm{C}(9)-\mathrm{C}(6) & 89.60(10) \\ \mathrm{C}(1)-\mathrm{C}(2)-\mathrm{C}(3) & 118.15(11) \\ \mathrm{C}(1)-\mathrm{C}(2)-\mathrm{C}(9) & 119.48(11) \\ \mathrm{C}(3)-\mathrm{C}(2)-\mathrm{C}(9) & 91.66(9) \\ \mathrm{O}(3)-\mathrm{C}(1)-\mathrm{O}(4) & 123.46(12) \\ \mathrm{O}(3)-\mathrm{C}(1)-\mathrm{C}(2) & 124.61(12) \\ \mathrm{O}(4)-\mathrm{C}(1)-\mathrm{C}(2) & 111.88(11) \\ \mathrm{O}(1)-\mathrm{C}(5)-\mathrm{C}(4) & 59.41(9) \\ \mathrm{O}(1)-\mathrm{C}(5)-\mathrm{C}(6) & 106.82(11) \\ \mathrm{C}(4)-\mathrm{C}(5)-\mathrm{C}(6) & 91.52(11) \\ \mathrm{O}(1)-\mathrm{C}(4)-\mathrm{C}(5) & 59.13(9) \\ \mathrm{O}(1)-\mathrm{C}(4)-\mathrm{C}(3) & 105.54(10) \\ \mathrm{C}(5)-\mathrm{C}(4)-\mathrm{C}(3) & 92.63(10) \\ \mathrm{O}(2)-\mathrm{C}(8)-\mathrm{C}(9) & 106.46(11) \\ & \end{array}$

Symmetry transformations used to generate equivalent atoms:

Table 4. Anisotropic displacement parameters $\left(\AA^{2} \times 10^{3}\right)$ for Compound 6. The anisotropic displacement factor exponent takes the form: $-2 p^{2}\left[h^{2} a^{* 2} U^{11}+\ldots+2 h k a^{*} b^{*} U^{12}\right]$

\begin{tabular}{lcccccc}
\hline & $\mathrm{U}^{11}$ & $\mathrm{U}^{22}$ & $\mathrm{U}^{33}$ & $\mathrm{U}^{23}$ & $\mathrm{U}^{13}$ & $\mathrm{U}^{12}$ \\
\hline $\mathrm{O}(2)$ & $45(1)$ & $29(1)$ & $30(1)$ & $8(1)$ & $-1(1)$ & $-6(1)$ \\
$\mathrm{O}(1)$ & $38(1)$ & $35(1)$ & $42(1)$ & $3(1)$ & $-6(1)$ & $-12(1)$ \\
$\mathrm{O}(4)$ & $27(1)$ & $53(1)$ & $25(1)$ & $7(1)$ & $-2(1)$ & $-2(1)$ \\
$\mathrm{C}(7)$ & $33(1)$ & $29(1)$ & $25(1)$ & $4(1)$ & $-2(1)$ & $-1(1)$ \\
$\mathrm{C}(3)$ & $23(1)$ & $25(1)$ & $24(1)$ & $2(1)$ & $2(1)$ & $2(1)$ \\
$\mathrm{C}(6)$ & $25(1)$ & $23(1)$ & $22(1)$ & $1(1)$ & $0(1)$ & $2(1)$ \\
$\mathrm{C}(9)$ & $24(1)$ & $34(1)$ & $22(1)$ & $3(1)$ & $3(1)$ & $2(1)$ \\
$\mathrm{C}(2)$ & $25(1)$ & $23(1)$ & $23(1)$ & $2(1)$ & $1(1)$ & $0(1)$ \\
$\mathrm{O}(3)$ & $35(1)$ & $84(1)$ & $35(1)$ & $27(1)$ & $1(1)$ & $-6(1)$ \\
$\mathrm{C}(1)$ & $28(1)$ & $26(1)$ & $23(1)$ & $1(1)$ & $2(1)$ & $0(1)$ \\
$\mathrm{C}(5)$ & $38(1)$ & $25(1)$ & $32(1)$ & $1(1)$ & $-1(1)$ & $1(1)$ \\
$\mathrm{C}(4)$ & $31(1)$ & $29(1)$ & $30(1)$ & $6(1)$ & $-1(1)$ & $-4(1)$ \\
$\mathrm{C}(8)$ & $36(1)$ & $51(1)$ & $28(1)$ & $9(1)$ & $2(1)$ & $-14(1)$ \\
$\mathrm{C}(10)$ & $36(1)$ & $52(1)$ & $29(1)$ & $3(1)$ & $-8(1)$ & $5(1)$ \\
& & & & & & \\
\hline
\end{tabular}

Table 5. Hydrogen coordinates $\left(\mathrm{x} 10^{4}\right)$ and isotropic displacement parameters $\left(\AA^{2} \mathrm{x} 10^{3}\right)$ for Compound 6.

$\begin{array}{llll}\mathrm{x} & \mathrm{y} & \mathrm{z} & \mathrm{U}(\mathrm{eq})\end{array}$




\begin{tabular}{lrrrr}
$\mathrm{H}(7 \mathrm{~A})$ & 5130 & 5730 & 4309 & 35 \\
$\mathrm{H}(7 \mathrm{~B})$ & 3024 & 6330 & 4793 & 35 \\
$\mathrm{H}(3 \mathrm{~A})$ & 5272 & 5760 & 2344 & 29 \\
$\mathrm{H}(9 \mathrm{~A})$ & -479 & 6835 & 2929 & 32 \\
$\mathrm{H}(2 \mathrm{~A})$ & 1788 & 4877 & 1997 & 28 \\
$\mathrm{H}(5 \mathrm{~A})$ & 2527 & 8712 & 3355 & 38 \\
$\mathrm{H}(4 \mathrm{~A})$ & 4010 & 8231 & 1675 & 36 \\
$\mathrm{H}(8 \mathrm{~A})$ & -776 & 5376 & 4133 & 46 \\
$\mathrm{H}(8 \mathrm{~B})$ & -447 & 4389 & 3297 & 46 \\
$\mathrm{H}(10 \mathrm{~A})$ & -4254 & 6413 & 268 & 59 \\
$\mathrm{H}(10 \mathrm{~B})$ & -2124 & 6106 & -314 & 59 \\
$\mathrm{H}(10 \mathrm{C})$ & -2411 & 7462 & 141 & 59 \\
\hline
\end{tabular}

Table 6. Torsion angles $\left[{ }^{\circ}\right]$ for Compound 6.

\begin{tabular}{lc}
\hline $\mathrm{C}(8)-\mathrm{O}(2)-\mathrm{C}(7)-\mathrm{C}(6)$ & $-40.78(14)$ \\
$\mathrm{O}(2)-\mathrm{C}(7)-\mathrm{C}(6)-\mathrm{C}(5)$ & $169.19(13)$ \\
$\mathrm{O}(2)-\mathrm{C}(7)-\mathrm{C}(6)-\mathrm{C}(9)$ & $27.69(14)$ \\
$\mathrm{O}(2)-\mathrm{C}(7)-\mathrm{C}(6)-\mathrm{C}(3)$ & $-72.91(15)$ \\
$\mathrm{C}(4)-\mathrm{C}(3)-\mathrm{C}(6)-\mathrm{C}(7)$ & $-136.79(12)$ \\
$\mathrm{C}(2)-\mathrm{C}(3)-\mathrm{C}(6)-\mathrm{C}(7)$ & $105.92(12)$ \\
$\mathrm{C}(4)-\mathrm{C}(3)-\mathrm{C}(6)-\mathrm{C}(5)$ & $-1.99(10)$ \\
$\mathrm{C}(2)-\mathrm{C}(3)-\mathrm{C}(6)-\mathrm{C}(5)$ & $-119.28(10)$ \\
$\mathrm{C}(4)-\mathrm{C}(3)-\mathrm{C}(6)-\mathrm{C}(9)$ & $114.20(9)$ \\
$\mathrm{C}(2)-\mathrm{C}(3)-\mathrm{C}(6)-\mathrm{C}(9)$ & $-3.09(9)$ \\
$\mathrm{C}(7)-\mathrm{C}(6)-\mathrm{C}(9)-\mathrm{C}(8)$ & $-5.30(14)$ \\
$\mathrm{C}(5)-\mathrm{C}(6)-\mathrm{C}(9)-\mathrm{C}(8)$ & $-151.46(13)$ \\
$\mathrm{C}(3)-\mathrm{C}(6)-\mathrm{C}(9)-\mathrm{C}(8)$ & $119.77(11)$ \\
$\mathrm{C}(7)-\mathrm{C}(6)-\mathrm{C}(9)-\mathrm{C}(2)$ & $-122.01(11)$ \\
$\mathrm{C}(5)-\mathrm{C}(6)-\mathrm{C}(9)-\mathrm{C}(2)$ & $91.83(13)$ \\
$\mathrm{C}(3)-\mathrm{C}(6)-\mathrm{C}(9)-\mathrm{C}(2)$ & $3.07(9)$ \\
$\mathrm{C}(4)-\mathrm{C}(3)-\mathrm{C}(2)-\mathrm{C}(1)$ & $42.35(16)$ \\
$\mathrm{C}(6)-\mathrm{C}(3)-\mathrm{C}(2)-\mathrm{C}(1)$ & $128.12(11)$ \\
$\mathrm{C}(4)-\mathrm{C}(3)-\mathrm{C}(2)-\mathrm{C}(9)$ & $-82.67(12)$ \\
$\mathrm{C}(6)-\mathrm{C}(3)-\mathrm{C}(2)-\mathrm{C}(9)$ & $3.10(9)$ \\
$\mathrm{C}(8)-\mathrm{C}(9)-\mathrm{C}(2)-\mathrm{C}(1)$ & $129.65(12)$ \\
$\mathrm{C}(6)-\mathrm{C}(9)-\mathrm{C}(2)-\mathrm{C}(1)$ & $-127.11(11)$ \\
$\mathrm{C}(8)-\mathrm{C}(9)-\mathrm{C}(2)-\mathrm{C}(3)$ & $-106.40(11)$ \\
$\mathrm{C}(6)-\mathrm{C}(9)-\mathrm{C}(2)-\mathrm{C}(3)$ & $-3.16(9)$ \\
$\mathrm{C}(10)-\mathrm{O}(4)-\mathrm{C}(1)-\mathrm{O}(3)$ & $-1.4(2)$ \\
$\mathrm{C}(10)-\mathrm{O}(4)-\mathrm{C}(1)-\mathrm{C}(2)$ & $-179.20(11)$ \\
$\mathrm{C}(3)-\mathrm{C}(2)-\mathrm{C}(1)-\mathrm{O}(3)$ & $24.6(2)$ \\
$\mathrm{C}(9)-\mathrm{C}(2)-\mathrm{C}(1)-\mathrm{O}(3)$ & $134.49(15)$ \\
$\mathrm{C}(3)-\mathrm{C}(2)-\mathrm{C}(1)-\mathrm{O}(4)$ & $-157.62(11)$ \\
$\mathrm{C}(9)-\mathrm{C}(2)-\mathrm{C}(1)-\mathrm{O}(4)$ & $-47.74(16)$ \\
$\mathrm{C}(4)-\mathrm{O}(1)-\mathrm{C}(5)-\mathrm{C}(6)$ & $81.57(11)$ \\
$\mathrm{C}(7)-\mathrm{C}(6)-\mathrm{C}(5)-\mathrm{O}(1)$ & $76.25(17)$ \\
$\mathrm{C}(9)-\mathrm{C}(6)-\mathrm{C}(5)-\mathrm{O}(1)$ & $-145.60(11)$ \\
$\mathrm{C}(3)-\mathrm{C}(6)-\mathrm{C}(5)-\mathrm{O}(1)$ & $-56.36(11)$ \\
$\mathrm{C}(7)-\mathrm{C}(6)-\mathrm{C}(5)-\mathrm{C}(4)$ & $134.66(13)$ \\
$\mathrm{C}(9)-\mathrm{C}(6)-\mathrm{C}(5)-\mathrm{C}(4)$ & $-87.19(13)$ \\
$\mathrm{C}(3)-\mathrm{C}(6)-\mathrm{C}(5)-\mathrm{C}(4)$ & $2.05(10)$ \\
& \\
& \\
&
\end{tabular}


$\mathrm{C}(5)-\mathrm{O}(1)-\mathrm{C}(4)-\mathrm{C}(3)$

$-83.64(11)$

$\mathrm{C}(6)-\mathrm{C}(5)-\mathrm{C}(4)-\mathrm{O}(1)$

$-108.70(10)$

$\mathrm{O}(1)-\mathrm{C}(5)-\mathrm{C}(4)-\mathrm{C}(3)$

$106.56(10)$

$\mathrm{C}(6)-\mathrm{C}(5)-\mathrm{C}(4)-\mathrm{C}(3)$

$-2.14(10)$

$\mathrm{C}(2)-\mathrm{C}(3)-\mathrm{C}(4)-\mathrm{O}(1)$

$147.68(11)$

$\mathrm{C}(6)-\mathrm{C}(3)-\mathrm{C}(4)-\mathrm{O}(1)$

$60.67(11)$

$\mathrm{C}(2)-\mathrm{C}(3)-\mathrm{C}(4)-\mathrm{C}(5)$

89.04(13)

$\mathrm{C}(6)-\mathrm{C}(3)-\mathrm{C}(4)-\mathrm{C}(5)$

2.03(10)

$\mathrm{C}(7)-\mathrm{O}(2)-\mathrm{C}(8)-\mathrm{C}(9)$

$38.09(15)$

$\mathrm{C}(2)-\mathrm{C}(9)-\mathrm{C}(8)-\mathrm{O}(2)$

$76.46(14)$

$\mathrm{C}(6)-\mathrm{C}(9)-\mathrm{C}(8)-\mathrm{O}(2)$

Symmetry transformations used to generate equivalent atoms:

\section{X-ray Crystal Structure of Compound IX}

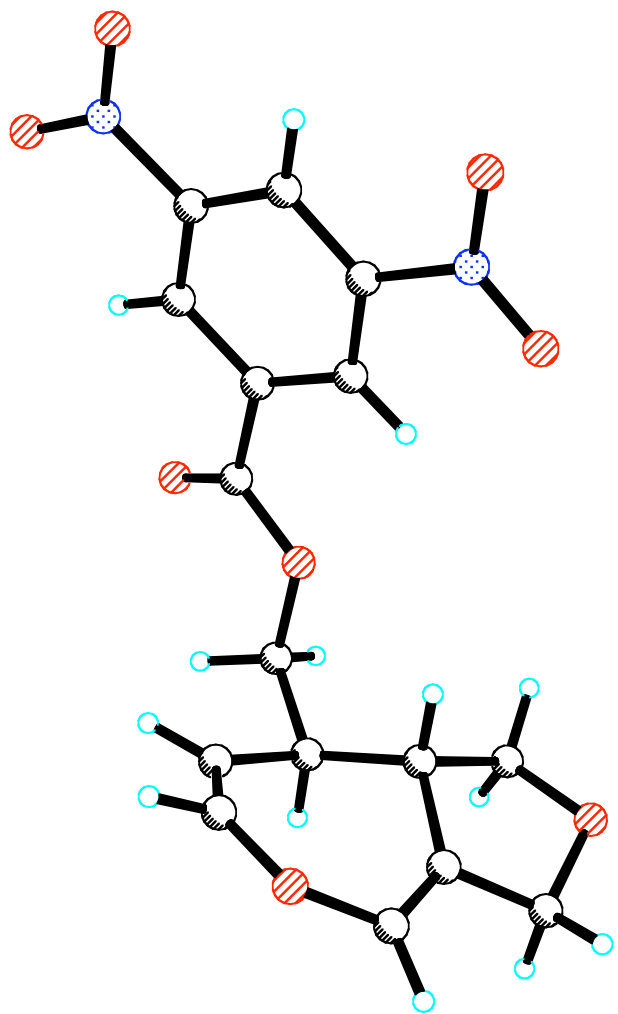

Table 1. Crystal data and structure refinement for Compound IX.

Identification code

Empirical formula

aj102t

Formula weight

Temperature

Wavelength

C14 H13 N2 O8

Crystal system

337.26

193(2) K

$0.71073 \AA$

Space group

Monoclinic

P2(1)/n 


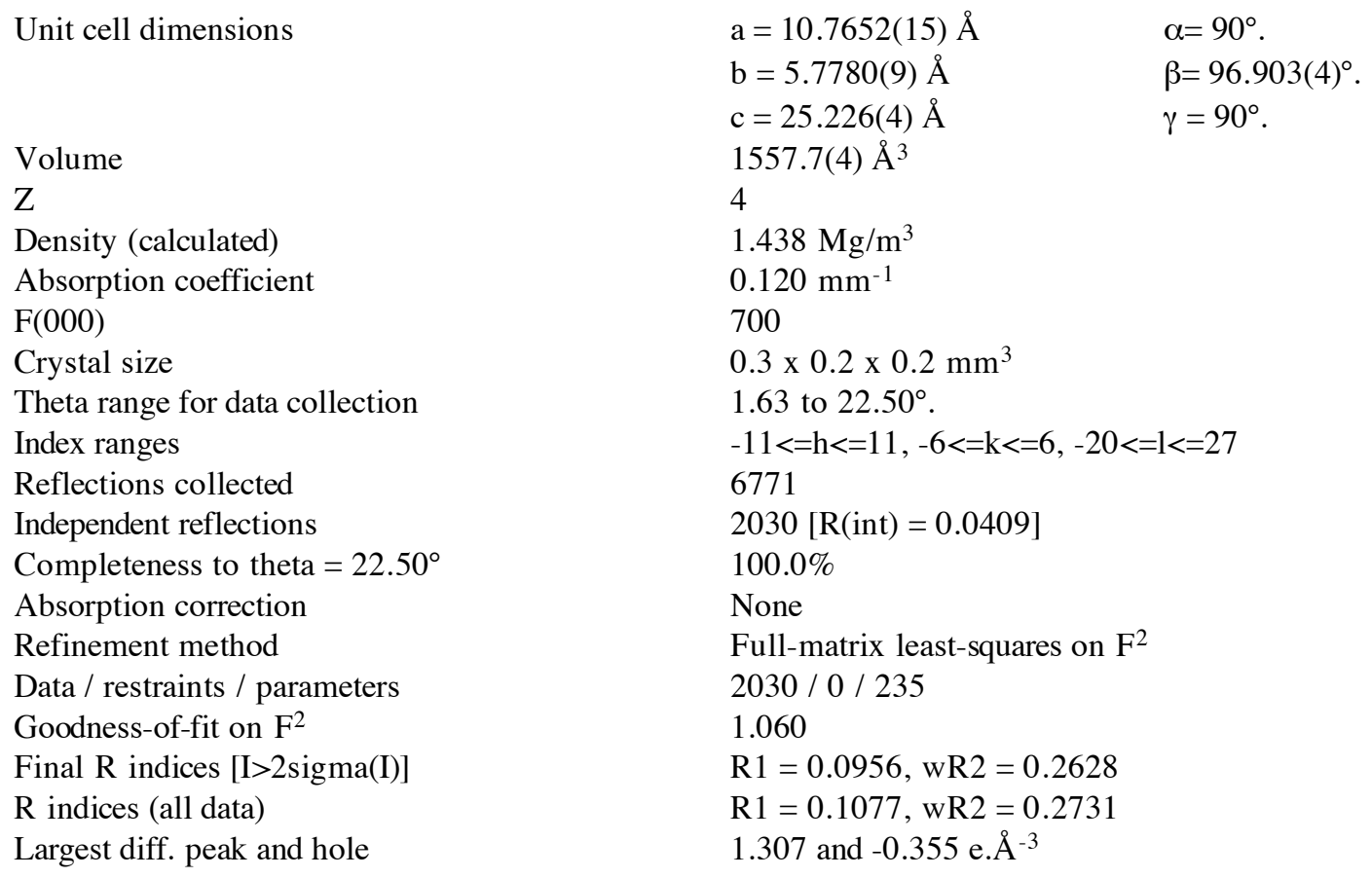

Table 2. Atomic coordinates ( $\left.\mathrm{x} 10^{4}\right)$ and equivalent isotropic displacement parameters $\left(\AA^{2} \times 10^{3}\right)$ for Compound IX. U(eq) is defined as one third of the trace of the orthogonalized $\mathrm{U}^{\mathrm{ij}}$ tensor.

\begin{tabular}{|c|c|c|c|c|}
\hline & $\mathrm{X}$ & $\mathrm{y}$ & $\mathrm{z}$ & $\mathrm{U}(\mathrm{eq})$ \\
\hline $\mathrm{O}(4)$ & $6026(4)$ & $10477(7)$ & $446(2)$ & $61(1)$ \\
\hline $\mathrm{O}(8)$ & $2831(4)$ & $4448(8)$ & $19(2)$ & $65(1)$ \\
\hline$C(15)$ & $4166(5)$ & $4411(10)$ & $804(2)$ & $44(1)$ \\
\hline $\mathrm{C}(11)$ & $5775(5)$ & $7211(9)$ & $990(2)$ & $38(1)$ \\
\hline$C(12)$ & $6165(5)$ & $6119(9)$ & $1476(2)$ & $41(1)$ \\
\hline$C(16)$ & $4758(5)$ & $6375(9)$ & $653(2)$ & $39(1)$ \\
\hline $\mathrm{C}(14)$ & $4517(5)$ & $3274(10)$ & $1281(2)$ & $46(1)$ \\
\hline $\mathrm{N}(17)$ & $5925(5)$ & 2994(9) & $2116(2)$ & $58(1)$ \\
\hline $\mathrm{O}(1)$ & $10939(4)$ & $11600(8)$ & $2403(2)$ & $69(1)$ \\
\hline$C(10)$ & $6446(5)$ & $9251(10)$ & $802(2)$ & $42(1)$ \\
\hline $\mathrm{O}(5)$ & $6841(4)$ & $3737(8)$ & $2389(2)$ & $65(1)$ \\
\hline $\mathrm{N}(18)$ & $3134(4)$ & $3428(9)$ & $443(2)$ & $53(1)$ \\
\hline $\mathrm{O}(3)$ & $7545(4)$ & $9569(8)$ & $1083(2)$ & $73(2)$ \\
\hline $\mathrm{C}(13)$ & $5513(5)$ & $4182(10)$ & $1603(2)$ & $46(1)$ \\
\hline $\mathrm{O}(2)$ & $11649(4)$ & $6292(8)$ & $1059(2)$ & $71(1)$ \\
\hline $\mathrm{O}(7)$ & $2646(4)$ & $1669(9)$ & $577(2)$ & $75(2)$ \\
\hline $\mathrm{C}(4)$ & $11419(5)$ & $9247(11)$ & $1702(2)$ & $52(2)$ \\
\hline $\mathrm{C}(1)$ & $10075(6)$ & 9997(13) & $1600(3)$ & $75(2)$ \\
\hline $\mathrm{O}(6)$ & $5312(5)$ & 1364(9) & $2230(2)$ & $93(2)$ \\
\hline $\mathrm{C}(6)$ & $10648(7)$ & $6950(13)$ & $730(3)$ & $67(2)$ \\
\hline $\mathrm{C}(2)$ & $9985(7)$ & $12174(11)$ & 2011(3) & $69(2)$ \\
\hline $\mathrm{C}(5)$ & $12114(6)$ & $7739(12)$ & $1472(2)$ & $64(2)$ \\
\hline $\mathrm{C}(8)$ & $9644(5)$ & $10705(10)$ & 1049(2) & $51(2)$ \\
\hline $\mathrm{C}(7)$ & $9833(8)$ & $8554(16)$ & $673(3)$ & $89(3)$ \\
\hline $\mathrm{C}(3)$ & $11934(8)$ & $10710(17)$ & $2156(3)$ & $93(3)$ \\
\hline
\end{tabular}


$\begin{array}{lllll}\mathrm{C}(9) & 8342(6) & 11382(14) & 899(4) & 95(3)\end{array}$

Table 3. Bond lengths $[\AA ̊]$ and angles $\left[^{\circ}\right]$ for Compound IX

\begin{tabular}{|c|c|}
\hline $\mathrm{O}(4)-\mathrm{C}(10)$ & $1.191(6)$ \\
\hline $\mathrm{O}(8)-\mathrm{N}(18)$ & $1.230(6)$ \\
\hline$C(15)-C(16)$ & $1.377(7)$ \\
\hline$C(15)-C(14)$ & $1.384(8)$ \\
\hline $\mathrm{C}(15)-\mathrm{N}(18)$ & $1.465(7)$ \\
\hline$C(11)-C(16)$ & $1.390(7)$ \\
\hline$C(11)-C(12)$ & $1.397(7)$ \\
\hline$C(11)-C(10)$ & $1.489(8)$ \\
\hline$C(12)-C(13)$ & $1.380(7)$ \\
\hline$C(14)-C(13)$ & $1.370(8)$ \\
\hline $\mathrm{N}(17)-\mathrm{O}(6)$ & $1.204(7)$ \\
\hline $\mathrm{N}(17)-\mathrm{O}(5)$ & $1.211(6)$ \\
\hline $\mathrm{N}(17)-\mathrm{C}(13)$ & $1.484(8)$ \\
\hline $\mathrm{O}(1)-\mathrm{C}(2)$ & $1.379(8)$ \\
\hline $\mathrm{O}(1)-\mathrm{C}(3)$ & $1.401(8)$ \\
\hline $\mathrm{C}(10)-\mathrm{O}(3)$ & $1.316(6)$ \\
\hline $\mathrm{N}(18)-\mathrm{O}(7)$ & $1.210(6)$ \\
\hline $\mathrm{O}(3)-\mathrm{C}(9)$ & $1.464(7)$ \\
\hline $\mathrm{O}(2)-\mathrm{C}(6)$ & $1.333(8)$ \\
\hline $\mathrm{O}(2)-\mathrm{C}(5)$ & $1.382(8)$ \\
\hline$C(4)-C(5)$ & $1.328(9)$ \\
\hline $\mathrm{C}(4)-\mathrm{C}(3)$ & $1.476(10)$ \\
\hline $\mathrm{C}(4)-\mathrm{C}(1)$ & $1.502(9)$ \\
\hline $\mathrm{C}(1)-\mathrm{C}(8)$ & $1.471(9)$ \\
\hline $\mathrm{C}(1)-\mathrm{C}(2)$ & $1.640(10)$ \\
\hline$C(6)-C(7)$ & $1.273(11)$ \\
\hline $\mathrm{C}(8)-\mathrm{C}(9)$ & $1.460(9)$ \\
\hline $\mathrm{C}(8)-\mathrm{C}(7)$ & $1.591(11)$ \\
\hline $\mathrm{C}(16)-\mathrm{C}(15)-\mathrm{C}(14)$ & $123.0(5)$ \\
\hline $\mathrm{C}(16)-\mathrm{C}(15)-\mathrm{N}(18)$ & $119.3(5)$ \\
\hline $\mathrm{C}(14)-\mathrm{C}(15)-\mathrm{N}(18)$ & $117.6(5)$ \\
\hline $\mathrm{C}(16)-\mathrm{C}(11)-\mathrm{C}(12)$ & $120.7(5)$ \\
\hline$C(16)-C(11)-C(10)$ & $117.2(5)$ \\
\hline $\mathrm{C}(12)-\mathrm{C}(11)-\mathrm{C}(10)$ & $122.0(5)$ \\
\hline $\mathrm{C}(13)-\mathrm{C}(12)-\mathrm{C}(11)$ & $117.6(5)$ \\
\hline$C(15)-C(16)-C(11)$ & $118.3(5)$ \\
\hline $\mathrm{C}(13)-\mathrm{C}(14)-\mathrm{C}(15)$ & $116.5(5)$ \\
\hline $\mathrm{O}(6)-\mathrm{N}(17)-\mathrm{O}(5)$ & $125.0(5)$ \\
\hline $\mathrm{O}(6)-\mathrm{N}(17)-\mathrm{C}(13)$ & $117.0(6)$ \\
\hline $\mathrm{O}(5)-\mathrm{N}(17)-\mathrm{C}(13)$ & $118.0(5)$ \\
\hline $\mathrm{C}(2)-\mathrm{O}(1)-\mathrm{C}(3)$ & $108.2(5)$ \\
\hline $\mathrm{O}(4)-\mathrm{C}(10)-\mathrm{O}(3)$ & $123.9(5)$ \\
\hline $\mathrm{O}(4)-\mathrm{C}(10)-\mathrm{C}(11)$ & $123.8(5)$ \\
\hline $\mathrm{O}(3)-\mathrm{C}(10)-\mathrm{C}(11)$ & $112.3(5)$ \\
\hline $\mathrm{O}(7)-\mathrm{N}(18)-\mathrm{O}(8)$ & $124.5(5)$ \\
\hline $\mathrm{O}(7)-\mathrm{N}(18)-\mathrm{C}(15)$ & $118.3(5)$ \\
\hline $\mathrm{O}(8)-\mathrm{N}(18)-\mathrm{C}(15)$ & $117.2(5)$ \\
\hline $\mathrm{C}(10)-\mathrm{O}(3)-\mathrm{C}(9)$ & $117.0(5)$ \\
\hline
\end{tabular}




$\begin{array}{ll}\mathrm{C}(14)-\mathrm{C}(13)-\mathrm{C}(12) & 123.8(5) \\ \mathrm{C}(14)-\mathrm{C}(13)-\mathrm{N}(17) & 118.1(5) \\ \mathrm{C}(12)-\mathrm{C}(13)-\mathrm{N}(17) & 118.1(5) \\ \mathrm{C}(6)-\mathrm{O}(2)-\mathrm{C}(5) & 119.3(6) \\ \mathrm{C}(5)-\mathrm{C}(4)-\mathrm{C}(3) & 122.4(6) \\ \mathrm{C}(5)-\mathrm{C}(4)-\mathrm{C}(1) & 134.1(6) \\ \mathrm{C}(3)-\mathrm{C}(4)-\mathrm{C}(1) & 103.4(6) \\ \mathrm{C}(8)-\mathrm{C}(1)-\mathrm{C}(4) & 115.5(6) \\ \mathrm{C}(8)-\mathrm{C}(1)-\mathrm{C}(2) & 110.4(6) \\ \mathrm{C}(4)-\mathrm{C}(1)-\mathrm{C}(2) & 104.0(5) \\ \mathrm{C}(7)-\mathrm{C}(6)-\mathrm{O}(2) & 140.5(8) \\ \mathrm{O}(1)-\mathrm{C}(2)-\mathrm{C}(1) & 100.0(5) \\ \mathrm{C}(4)-\mathrm{C}(5)-\mathrm{O}(2) & 123.7(6) \\ \mathrm{C}(9)-\mathrm{C}(8)-\mathrm{C}(1) & 120.2(6) \\ \mathrm{C}(9)-\mathrm{C}(8)-\mathrm{C}(7) & 104.1(6) \\ \mathrm{C}(1)-\mathrm{C}(8)-\mathrm{C}(7) & 107.3(5) \\ \mathrm{C}(6)-\mathrm{C}(7)-\mathrm{C}(8) & 129.7(7) \\ \mathrm{O}(1)-\mathrm{C}(3)-\mathrm{C}(4) & 108.6(6) \\ \mathrm{O}(3)-\mathrm{C}(9)-\mathrm{C}(8) & 107.9(5)\end{array}$

Symmetry transformations used to generate equivalent atoms:

Table 4. Anisotropic displacement parameters $\left(\AA^{2} \times 10^{3}\right)$ for Compound IX. The anisotropic displacement factor exponent takes the form: $-2 \pi^{2}\left[h^{2} a^{* 2} U^{11}+\ldots+2 h k^{*} b^{*} U^{12}\right]$

\begin{tabular}{|c|c|c|c|c|c|c|}
\hline & $\mathrm{U}^{11}$ & $\mathrm{U}^{22}$ & $\mathrm{U}^{33}$ & $\mathrm{U}^{23}$ & $\mathrm{U}^{13}$ & $\mathrm{U}^{12}$ \\
\hline $\mathrm{O}(4)$ & $57(3)$ & $56(2)$ & $64(3)$ & $20(2)$ & $-15(2)$ & $-15(2)$ \\
\hline $\mathrm{O}(8)$ & $66(3)$ & $75(3)$ & $53(3)$ & $-8(2)$ & $0(2)$ & $-20(2)$ \\
\hline$C(15)$ & $40(3)$ & $49(3)$ & $44(3)$ & $-6(3)$ & $9(2)$ & $-5(3)$ \\
\hline $\mathrm{C}(11)$ & $32(3)$ & $38(3)$ & $44(3)$ & $3(2)$ & $6(2)$ & $4(2)$ \\
\hline $\mathrm{C}(12)$ & $37(3)$ & $39(3)$ & $47(3)$ & 3(3) & $8(2)$ & $5(2)$ \\
\hline$C(16)$ & $39(3)$ & $44(3)$ & $35(3)$ & $-1(2)$ & $8(2)$ & $0(2)$ \\
\hline $\mathrm{C}(14)$ & $43(3)$ & $43(3)$ & $55(4)$ & 1(3) & 12(3) & $-5(3)$ \\
\hline $\mathrm{N}(17)$ & $59(3)$ & $56(3)$ & $61(3)$ & $15(3)$ & $10(3)$ & $7(3)$ \\
\hline $\mathrm{O}(1)$ & $55(3)$ & $83(3)$ & $67(3)$ & $-24(3)$ & $-6(2)$ & $11(2)$ \\
\hline $\mathrm{C}(10)$ & $33(3)$ & $48(3)$ & $44(3)$ & $5(3)$ & $-1(2)$ & $2(3)$ \\
\hline $\mathrm{O}(5)$ & $62(3)$ & $69(3)$ & $60(3)$ & $13(2)$ & $-9(2)$ & $-1(2)$ \\
\hline $\mathrm{N}(18)$ & $51(3)$ & $57(3)$ & $53(3)$ & $-10(3)$ & $9(2)$ & $-16(3)$ \\
\hline $\mathrm{O}(3)$ & $37(2)$ & $92(3)$ & $85(3)$ & $50(3)$ & $-19(2)$ & $-21(2)$ \\
\hline $\mathrm{C}(13)$ & $49(3)$ & $45(3)$ & $47(3)$ & 4(3) & 13(3) & 8(3) \\
\hline $\mathrm{O}(2)$ & $66(3)$ & 84(3) & $61(3)$ & $-18(3)$ & 4(2) & $18(3)$ \\
\hline $\mathrm{O}(7)$ & $81(3)$ & $72(3)$ & $73(3)$ & $-4(2)$ & $7(2)$ & $-42(3)$ \\
\hline $\mathrm{C}(4)$ & $38(3)$ & $59(4)$ & $60(4)$ & 6(3) & 8(3) & $12(3)$ \\
\hline $\mathrm{C}(1)$ & $60(4)$ & 71(5) & $91(5)$ & 19(4) & $-1(4)$ & $-7(4)$ \\
\hline $\mathrm{O}(6)$ & $95(4)$ & $83(4)$ & $95(4)$ & $49(3)$ & $-9(3)$ & $-28(3)$ \\
\hline$C(6)$ & $60(4)$ & $62(4)$ & $74(5)$ & $5(4)$ & $-8(4)$ & 1(4) \\
\hline $\mathrm{C}(2)$ & $72(4)$ & $41(3)$ & $96(5)$ & $-8(3)$ & $15(4)$ & $-9(3)$ \\
\hline$C(5)$ & $64(4)$ & $74(4)$ & $50(4)$ & $5(3)$ & $-11(3)$ & $-18(4)$ \\
\hline $\mathrm{C}(8)$ & $37(3)$ & $50(3)$ & 63(4) & 18(3) & $-7(3)$ & $-10(3)$ \\
\hline$C(7)$ & $91(6)$ & $115(7)$ & $58(4)$ & $4(5)$ & $-8(4)$ & $-35(6)$ \\
\hline $\mathrm{C}(3)$ & $80(5)$ & $122(7)$ & $76(5)$ & $-16(5)$ & $2(4)$ & $41(5)$ \\
\hline $\mathrm{C}(9)$ & $40(4)$ & $99(6)$ & $138(7)$ & $73(6)$ & $-13(4)$ & $-20(4)$ \\
\hline
\end{tabular}


Table 5. Hydrogen coordinates $\left(\mathrm{x} 10^{4}\right)$ and isotropic displacement parameters $\left(\AA^{2} \mathrm{x} 10^{3}\right)$ for Compound IX.

\begin{tabular}{lrrrr}
\hline & $x$ & $y$ & $z$ & $U(e q)$ \\
\hline & & & & \\
$H(12 A)$ & 6855 & 6689 & 1710 & 49 \\
$H(16 A)$ & 4479 & 7137 & 326 & 47 \\
H(14A) & 4089 & 1933 & 1380 & 55 \\
H(1A) & 9530 & 8711 & 1703 & 90 \\
H(6A) & 10494 & 5870 & 446 & 80 \\
$\mathrm{H}(2 \mathrm{~A})$ & 10142 & 13673 & 1841 & 83 \\
$\mathrm{H}(2 \mathrm{~B})$ & 9165 & 12226 & 2151 & 83 \\
$\mathrm{H}(5 \mathrm{~A})$ & 12980 & 7648 & 1598 & 77 \\
$\mathrm{H}(8 \mathrm{~A})$ & 10192 & 11995 & 951 & 61 \\
$\mathrm{H}(7 \mathrm{~A})$ & 9250 & 8452 & 360 & 107 \\
$\mathrm{H}(3 \mathrm{~A})$ & 12492 & 9781 & 2414 & 112 \\
$\mathrm{H}(3 \mathrm{~B})$ & 12428 & 11994 & 2027 & 112 \\
$\mathrm{H}(9 \mathrm{~A})$ & 8172 & 12878 & 1068 & 113 \\
$\mathrm{H}(9 \mathrm{~B})$ & 8170 & 11559 & 507 & 113 \\
& & & & \\
\hline
\end{tabular}

Table 6. Torsion angles $\left[^{\circ}\right]$ for Compound IX.

\begin{tabular}{lc}
\hline $\mathrm{C}(16)-\mathrm{C}(11)-\mathrm{C}(12)-\mathrm{C}(13)$ & $0.6(7)$ \\
$\mathrm{C}(10)-\mathrm{C}(11)-\mathrm{C}(12)-\mathrm{C}(13)$ & $-177.3(5)$ \\
$\mathrm{C}(14)-\mathrm{C}(15)-\mathrm{C}(16)-\mathrm{C}(11)$ & $1.8(8)$ \\
$\mathrm{N}(18)-\mathrm{C}(15)-\mathrm{C}(16)-\mathrm{C}(11)$ & $-176.9(5)$ \\
$\mathrm{C}(12)-\mathrm{C}(11)-\mathrm{C}(16)-\mathrm{C}(15)$ & $-1.6(7)$ \\
$\mathrm{C}(10)-\mathrm{C}(11)-\mathrm{C}(16)-\mathrm{C}(15)$ & $176.4(4)$ \\
$\mathrm{C}(16)-\mathrm{C}(15)-\mathrm{C}(14)-\mathrm{C}(13)$ & $-0.9(8)$ \\
$\mathrm{N}(18)-\mathrm{C}(15)-\mathrm{C}(14)-\mathrm{C}(13)$ & $177.8(5)$ \\
$\mathrm{C}(16)-\mathrm{C}(11)-\mathrm{C}(10)-\mathrm{O}(4)$ & $16.2(8)$ \\
$\mathrm{C}(12)-\mathrm{C}(11)-\mathrm{C}(10)-\mathrm{O}(4)$ & $-165.8(5)$ \\
$\mathrm{C}(16)-\mathrm{C}(11)-\mathrm{C}(10)-\mathrm{O}(3)$ & $-165.1(5)$ \\
$\mathrm{C}(12)-\mathrm{C}(11)-\mathrm{C}(10)-\mathrm{O}(3)$ & $12.9(7)$ \\
$\mathrm{C}(16)-\mathrm{C}(15)-\mathrm{N}(18)-\mathrm{O}(7)$ & $178.2(5)$ \\
$\mathrm{C}(14)-\mathrm{C}(15)-\mathrm{N}(18)-\mathrm{O}(7)$ & $-0.5(8)$ \\
$\mathrm{C}(16)-\mathrm{C}(15)-\mathrm{N}(18)-\mathrm{O}(8)$ & $-1.1(7)$ \\
$\mathrm{C}(14)-\mathrm{C}(15)-\mathrm{N}(18)-\mathrm{O}(8)$ & $-179.8(5)$ \\
$\mathrm{O}(4)-\mathrm{C}(10)-\mathrm{O}(3)-\mathrm{C}(9)$ & $-7.4(9)$ \\
$\mathrm{C}(11)-\mathrm{C}(10)-\mathrm{O}(3)-\mathrm{C}(9)$ & $173.9(6)$ \\
$\mathrm{C}(15)-\mathrm{C}(14)-\mathrm{C}(13)-\mathrm{C}(12)$ & $-0.2(8)$ \\
$\mathrm{C}(15)-\mathrm{C}(14)-\mathrm{C}(13)-\mathrm{N}(17)$ & $-179.4(5)$ \\
$\mathrm{C}(11)-\mathrm{C}(12)-\mathrm{C}(13)-\mathrm{C}(14)$ & $0.3(8)$ \\
$\mathrm{C}(11)-\mathrm{C}(12)-\mathrm{C}(13)-\mathrm{N}(17)$ & $179.5(4)$ \\
$\mathrm{O}(6)-\mathrm{N}(17)-\mathrm{C}(13)-\mathrm{C}(14)$ & $-3.7(8)$ \\
$\mathrm{O}(5)-\mathrm{N}(17)-\mathrm{C}(13)-\mathrm{C}(14)$ & $176.7(5)$ \\
$\mathrm{O}(6)-\mathrm{N}(17)-\mathrm{C}(13)-\mathrm{C}(12)$ & $177.1(5)$ \\
$\mathrm{O}(5)-\mathrm{N}(17)-\mathrm{C}(13)-\mathrm{C}(12)$ & $-2.5(8)$ \\
$\mathrm{C}(5)-\mathrm{C}(4)-\mathrm{C}(1)-\mathrm{C}(8)$ & $-51.8(10)$ \\
$\mathrm{C}(3)-\mathrm{C}(4)-\mathrm{C}(1)-\mathrm{C}(8)$ & $126.3(7)$ \\
&
\end{tabular}




$\begin{array}{lc}\mathrm{C}(5)-\mathrm{C}(4)-\mathrm{C}(1)-\mathrm{C}(2) & -172.9(7) \\ \mathrm{C}(3)-\mathrm{C}(4)-\mathrm{C}(1)-\mathrm{C}(2) & 5.3(7) \\ \mathrm{C}(5)-\mathrm{O}(2)-\mathrm{C}(6)-\mathrm{C}(7) & -6.4(14) \\ \mathrm{C}(3)-\mathrm{O}(1)-\mathrm{C}(2)-\mathrm{C}(1) & 39.4(7) \\ \mathrm{C}(8)-\mathrm{C}(1)-\mathrm{C}(2)-\mathrm{O}(1) & -151.2(5) \\ \mathrm{C}(4)-\mathrm{C}(1)-\mathrm{C}(2)-\mathrm{O}(1) & -26.8(6) \\ \mathrm{C}(3)-\mathrm{C}(4)-\mathrm{C}(5)-\mathrm{O}(2) & 177.7(7) \\ \mathrm{C}(1)-\mathrm{C}(4)-\mathrm{C}(5)-\mathrm{O}(2) & -4.4(12) \\ \mathrm{C}(6)-\mathrm{O}(2)-\mathrm{C}(5)-\mathrm{C}(4) & 27.8(9) \\ \mathrm{C}(4)-\mathrm{C}(1)-\mathrm{C}(8)-\mathrm{C}(9) & 177.6(6) \\ \mathrm{C}(2)-\mathrm{C}(1)-\mathrm{C}(8)-\mathrm{C}(9) & -64.8(8) \\ \mathrm{C}(4)-\mathrm{C}(1)-\mathrm{C}(8)-\mathrm{C}(7) & 59.2(7) \\ \mathrm{C}(2)-\mathrm{C}(1)-\mathrm{C}(8)-\mathrm{C}(7) & 176.8(5) \\ \mathrm{O}(2)-\mathrm{C}(6)-\mathrm{C}(7)-\mathrm{C}(8) & -2.7(16) \\ \mathrm{C}(9)-\mathrm{C}(8)-\mathrm{C}(7)-\mathrm{C}(6) & -158.8(8) \\ \mathrm{C}(1)-\mathrm{C}(8)-\mathrm{C}(7)-\mathrm{C}(6) & -30.4(10) \\ \mathrm{C}(2)-\mathrm{O}(1)-\mathrm{C}(3)-\mathrm{C}(4) & -39.3(8) \\ \mathrm{C}(5)-\mathrm{C}(4)-\mathrm{C}(3)-\mathrm{O}(1) & -163.4(6) \\ \mathrm{C}(1)-\mathrm{C}(4)-\mathrm{C}(3)-\mathrm{O}(1) & 18.2(8) \\ \mathrm{C}(10)-\mathrm{O}(3)-\mathrm{C}(9)-\mathrm{C}(8) & -150.0(6) \\ \mathrm{C}(1)-\mathrm{C}(8)-\mathrm{C}(9)-\mathrm{O}(3) & -50.5(10) \\ \mathrm{C}(7)-\mathrm{C}(8)-\mathrm{C}(9)-\mathrm{O}(3) & 69.5(8)\end{array}$

Symmetry transformations used to generate equivalent atoms: 


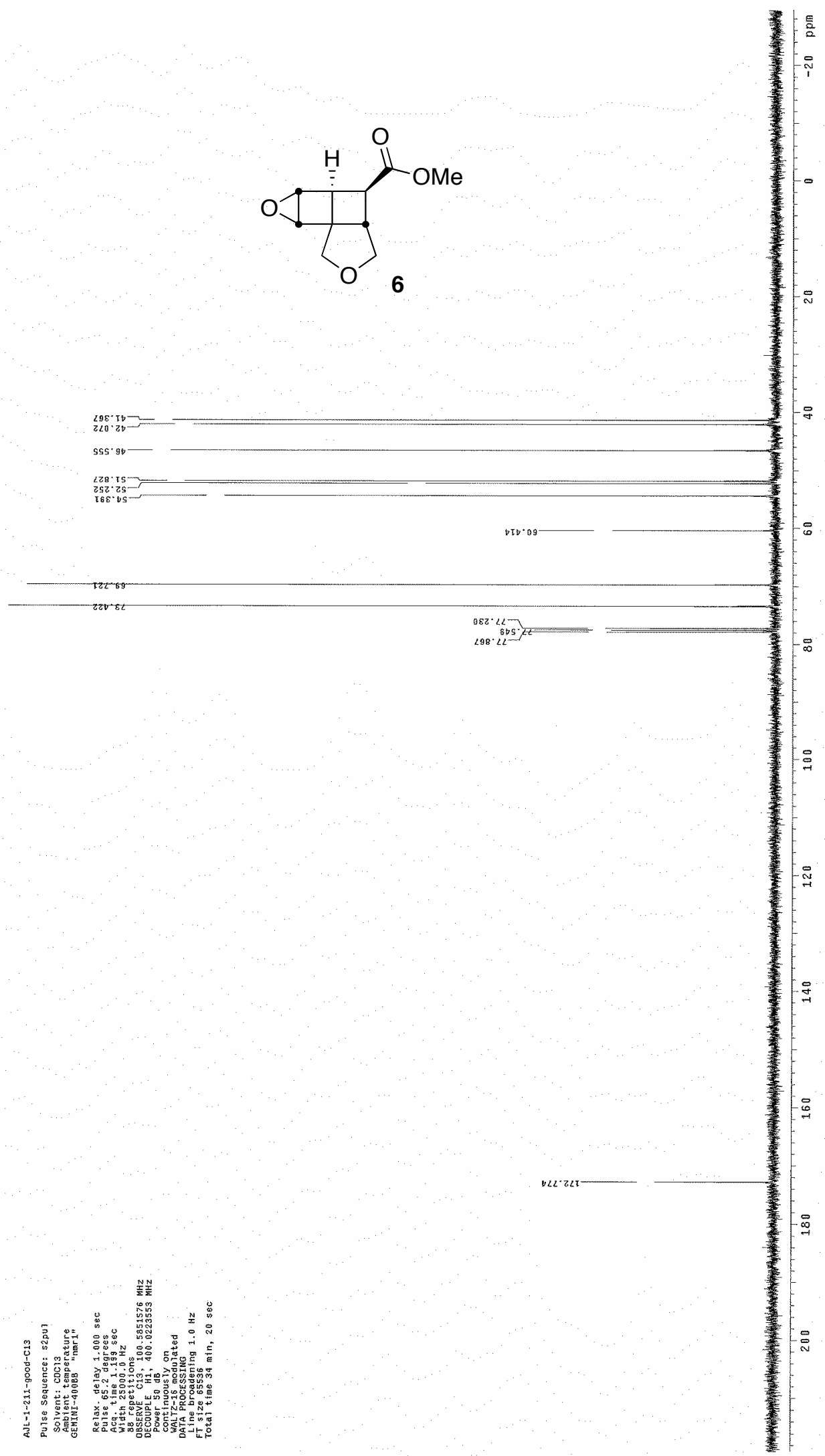



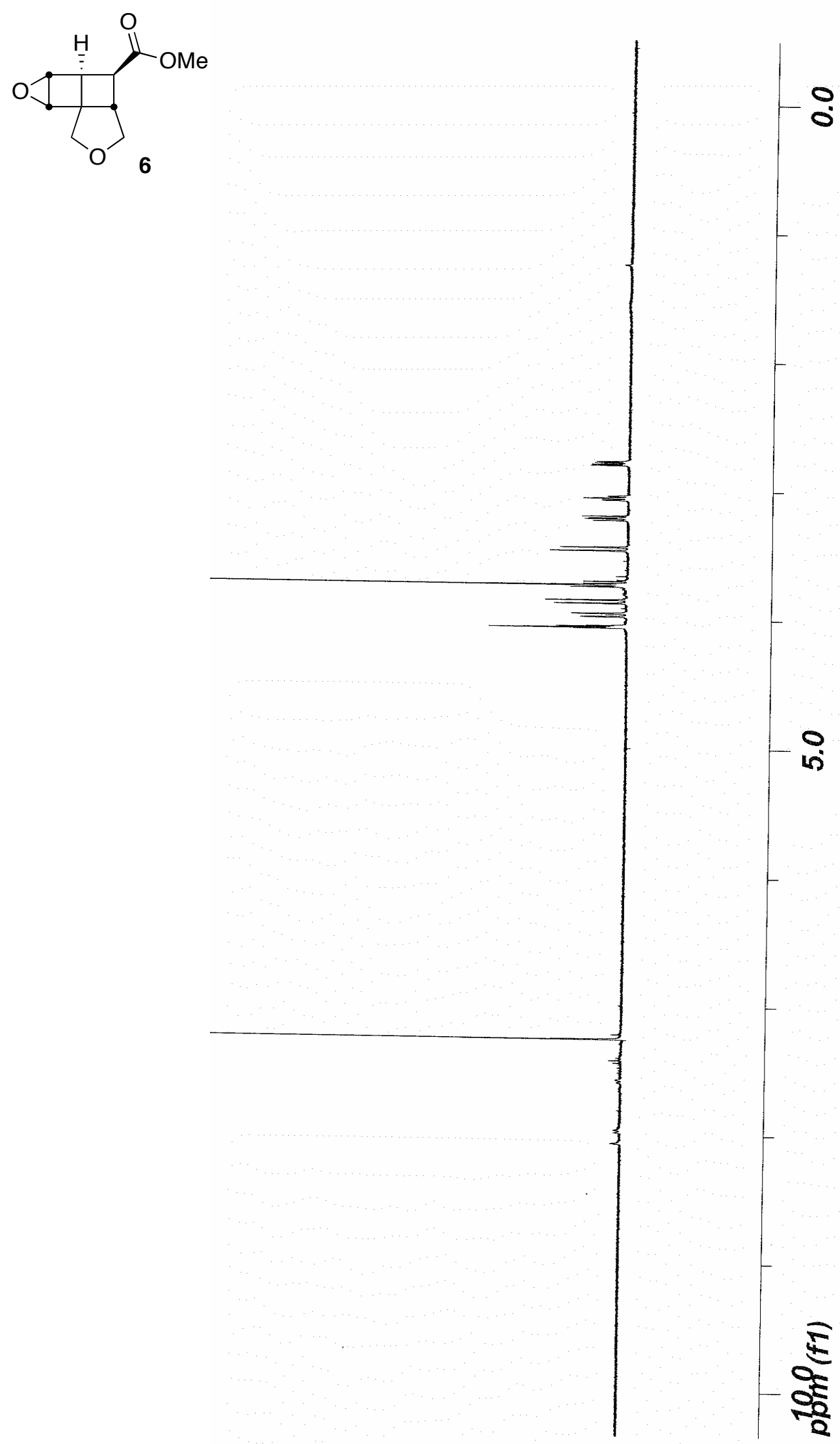


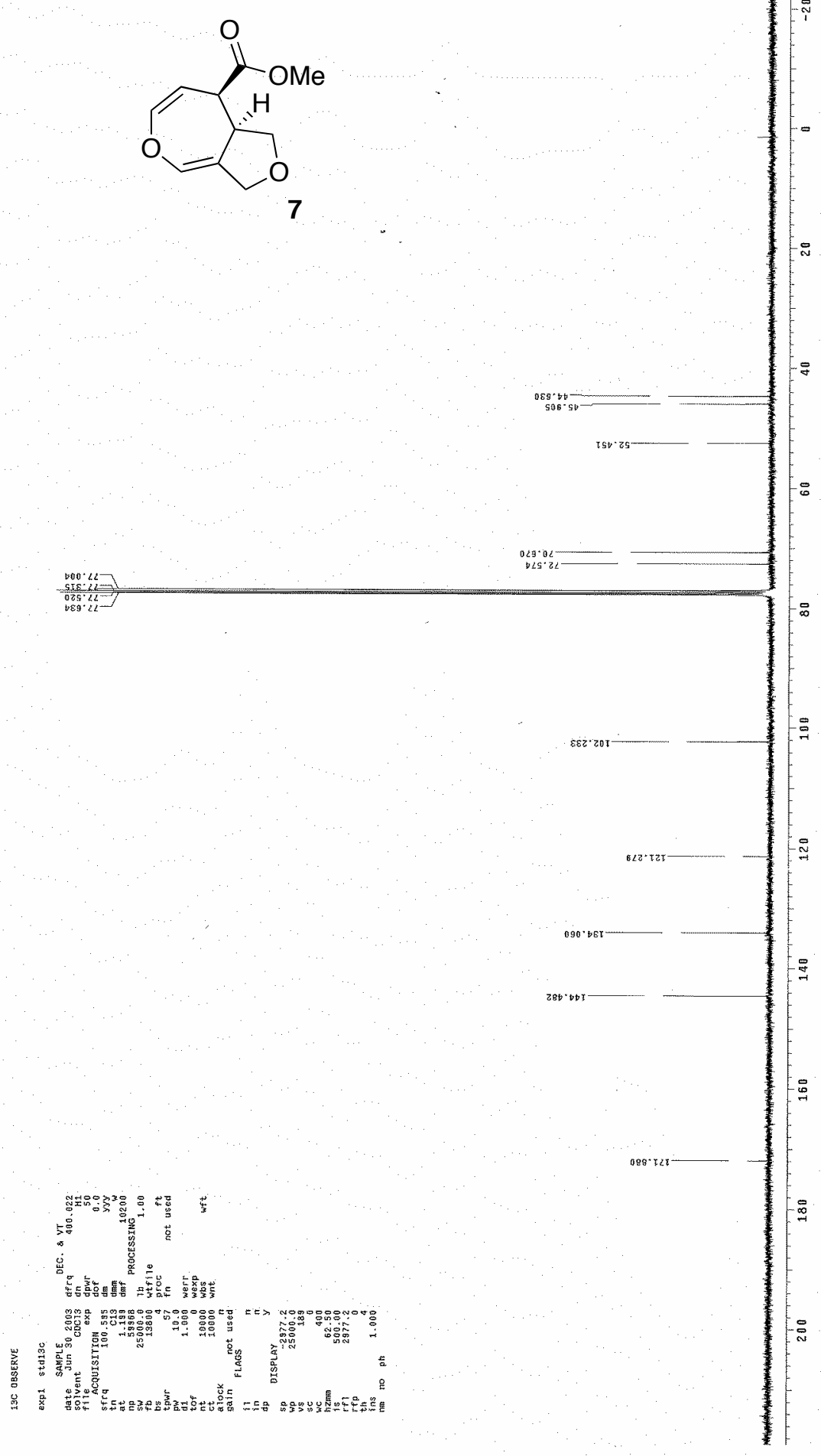




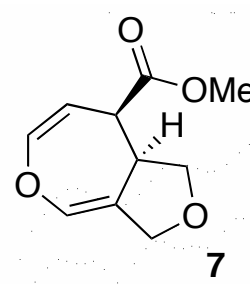

7

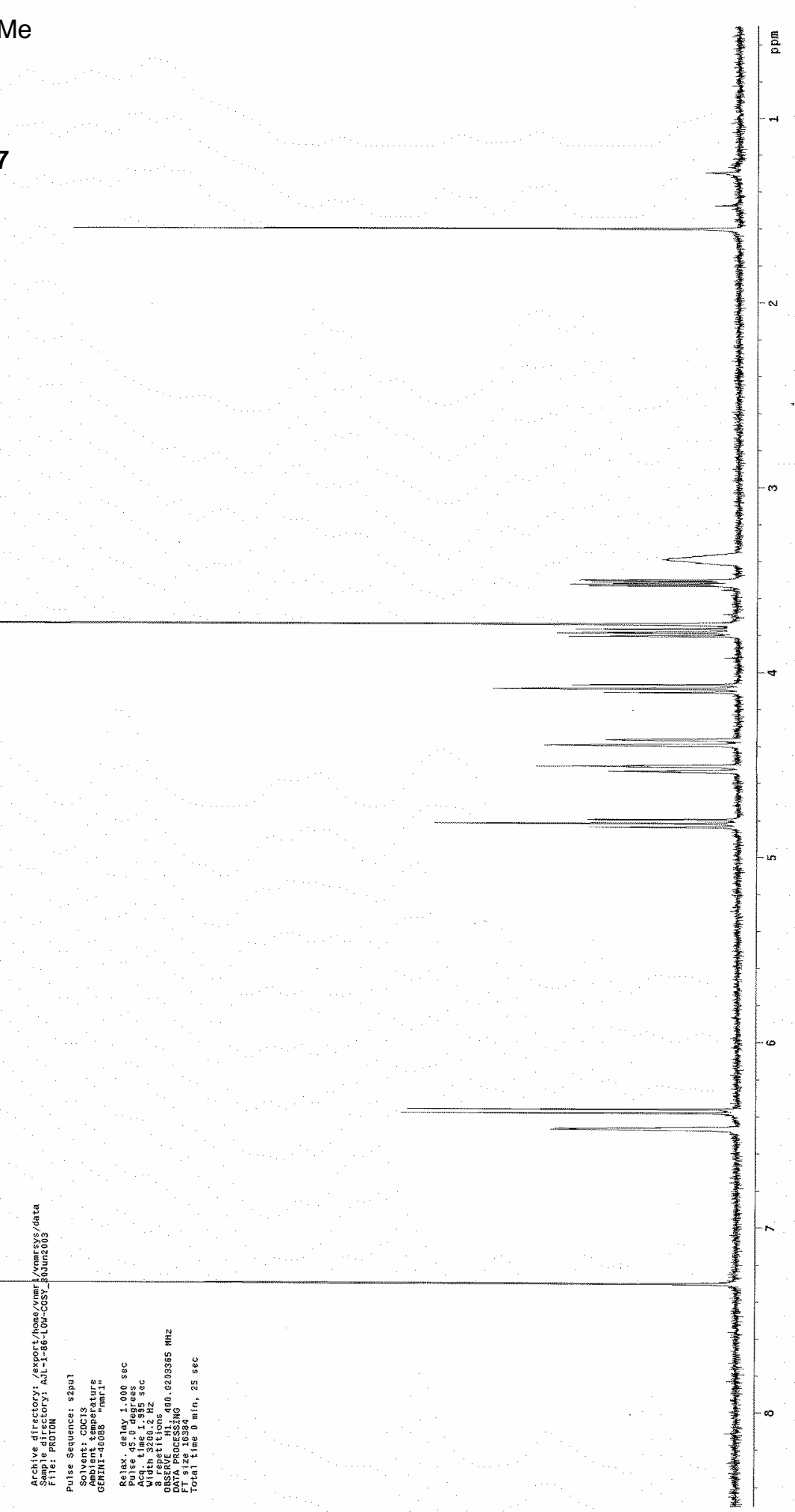




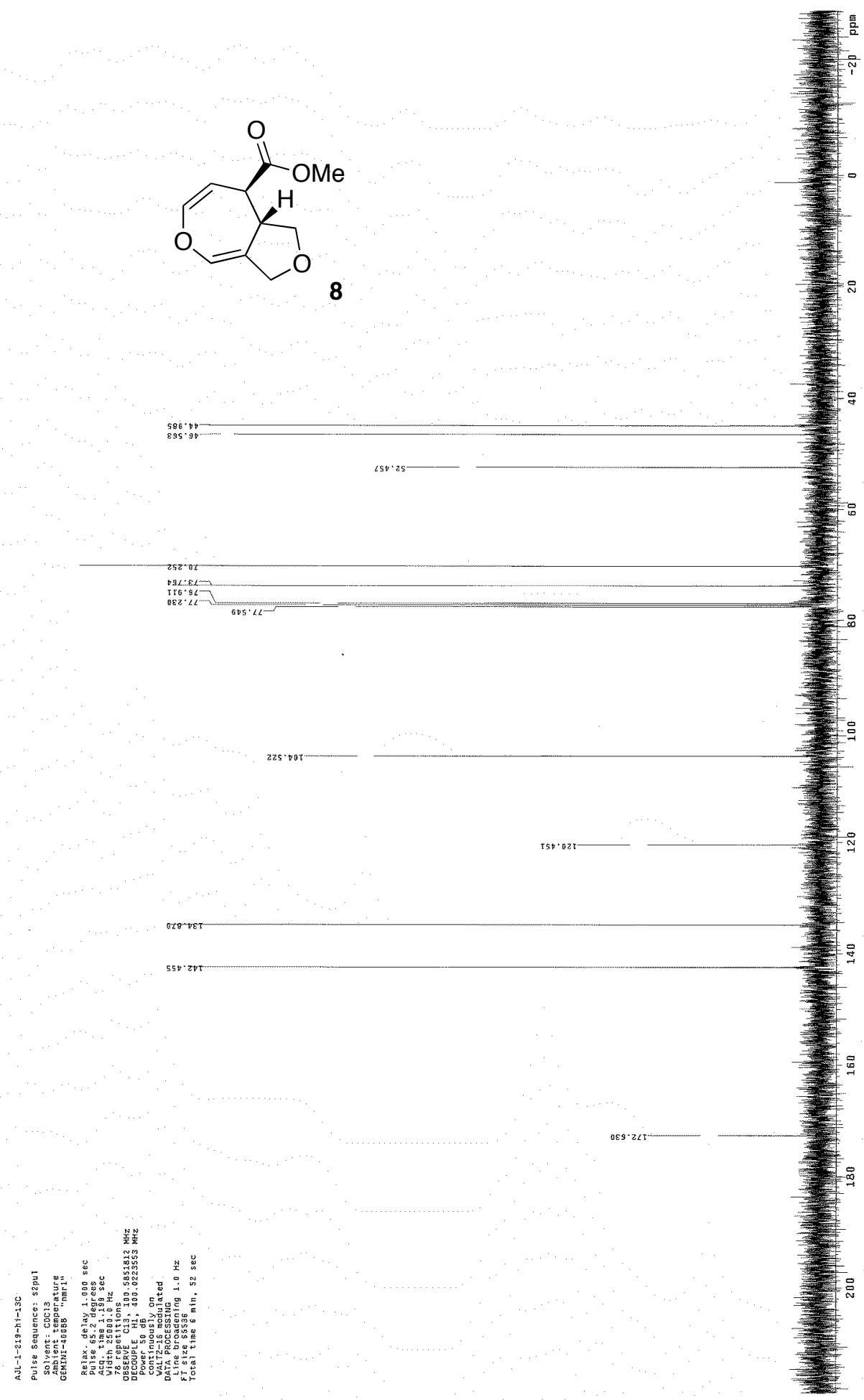




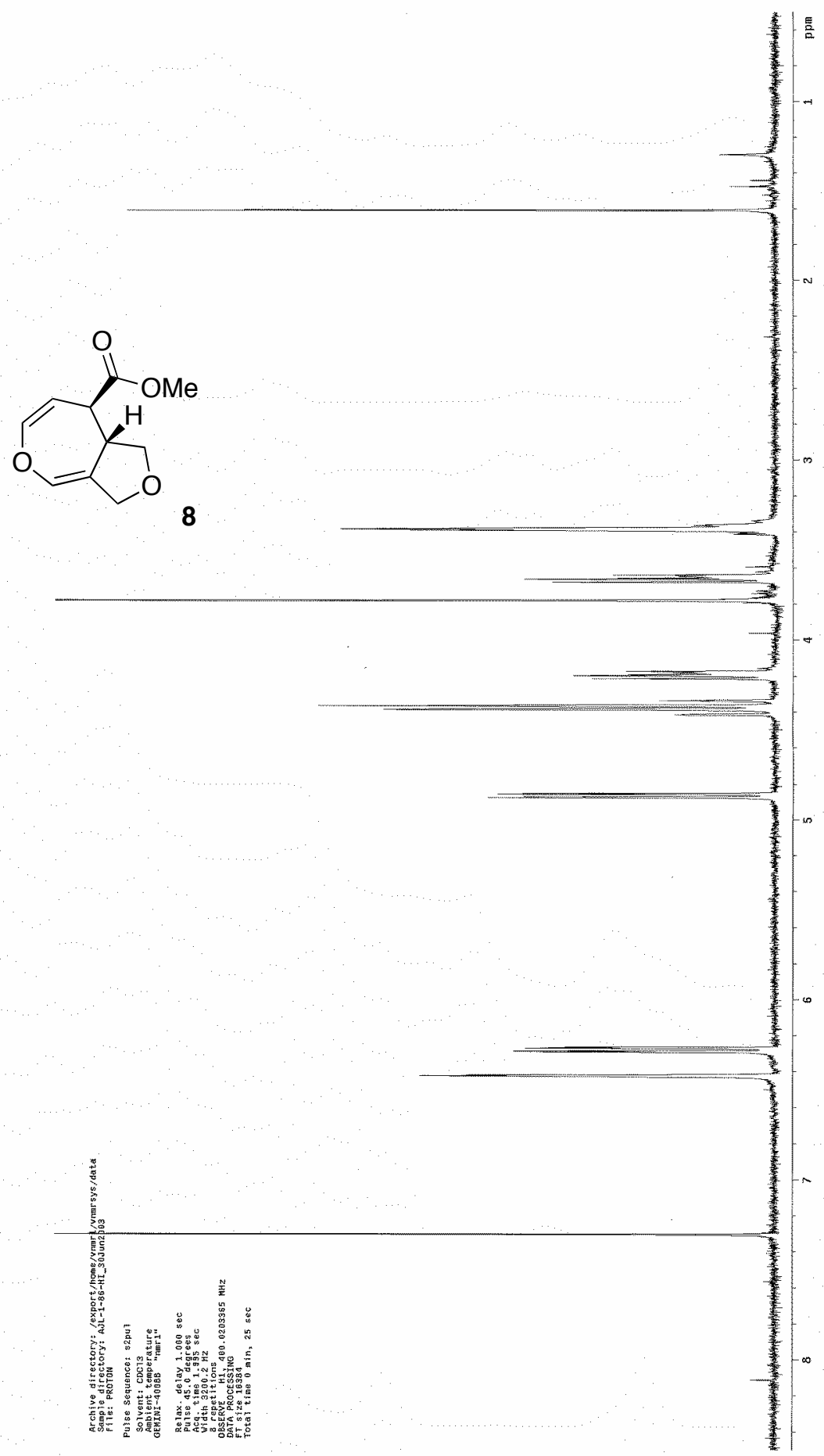




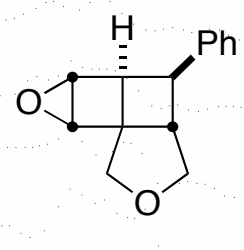

9
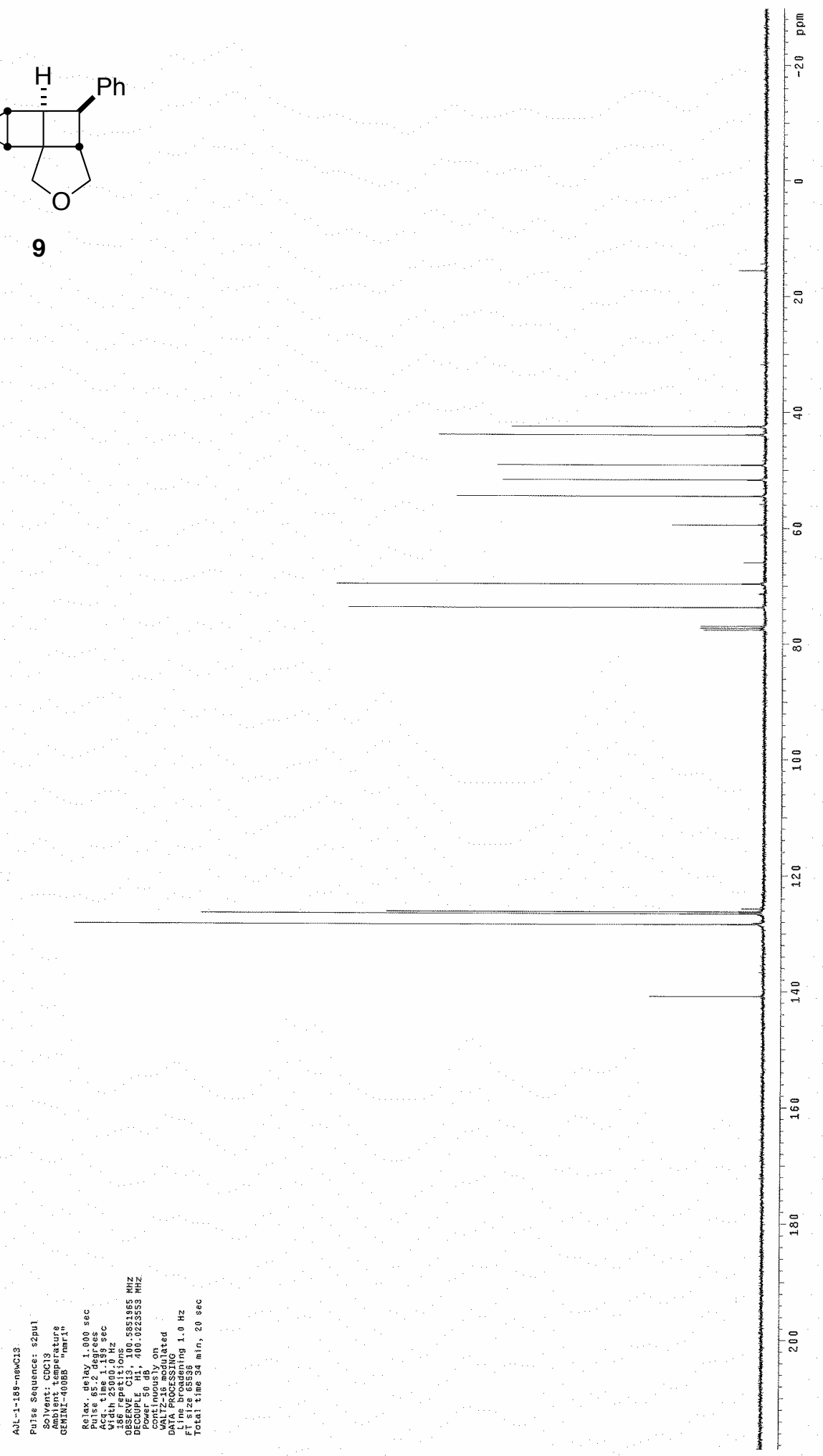


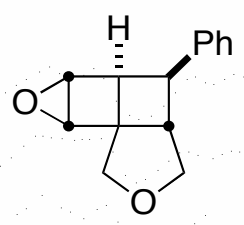

9

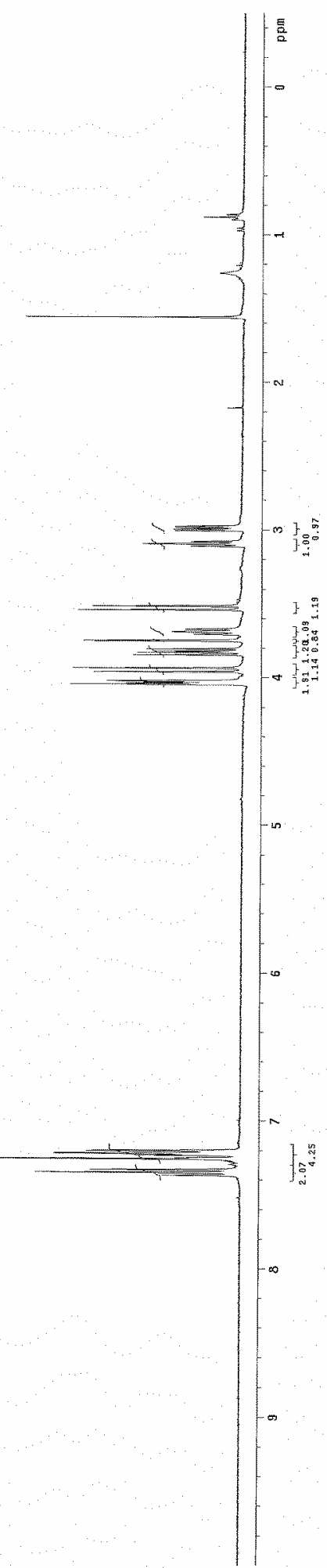



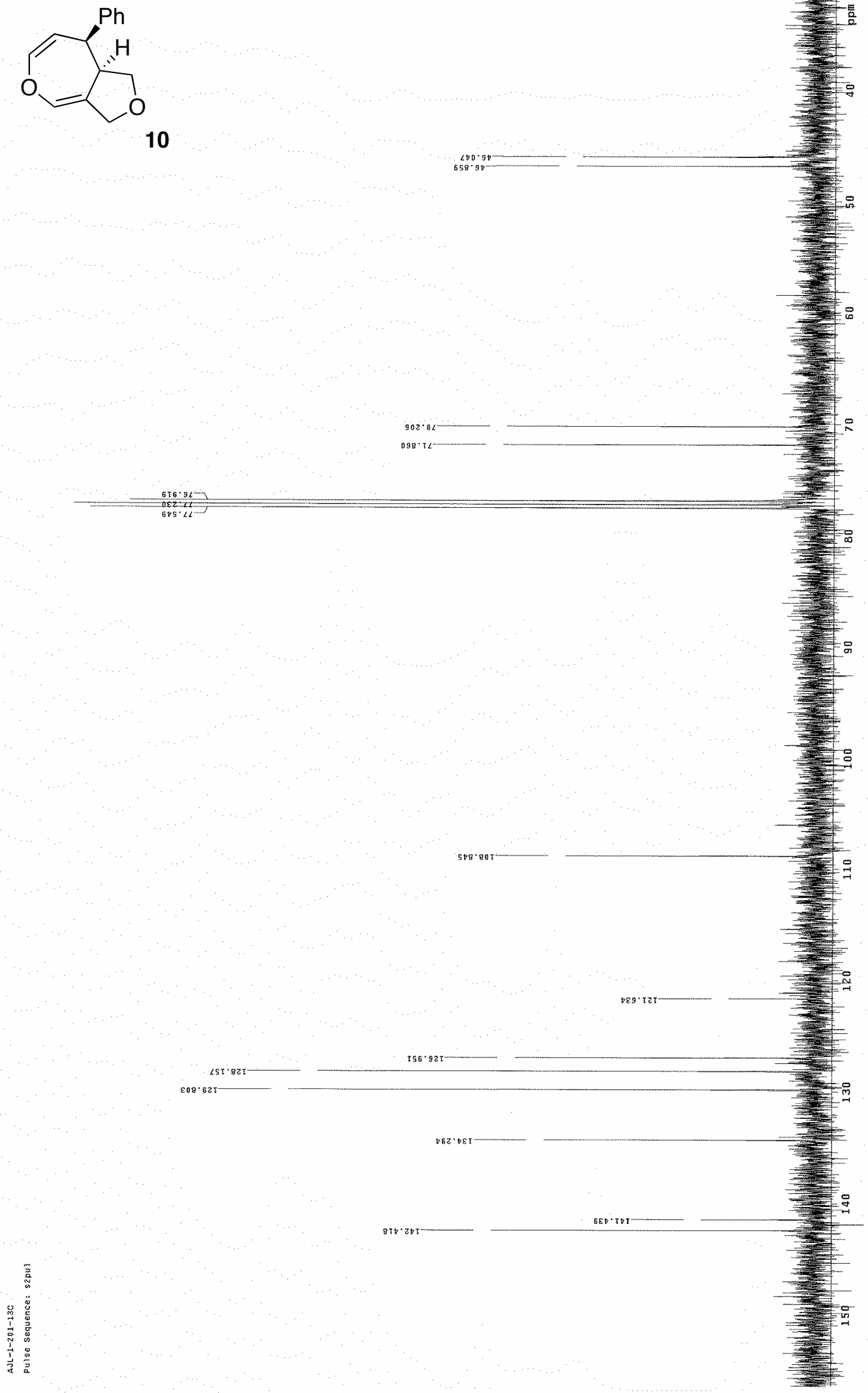


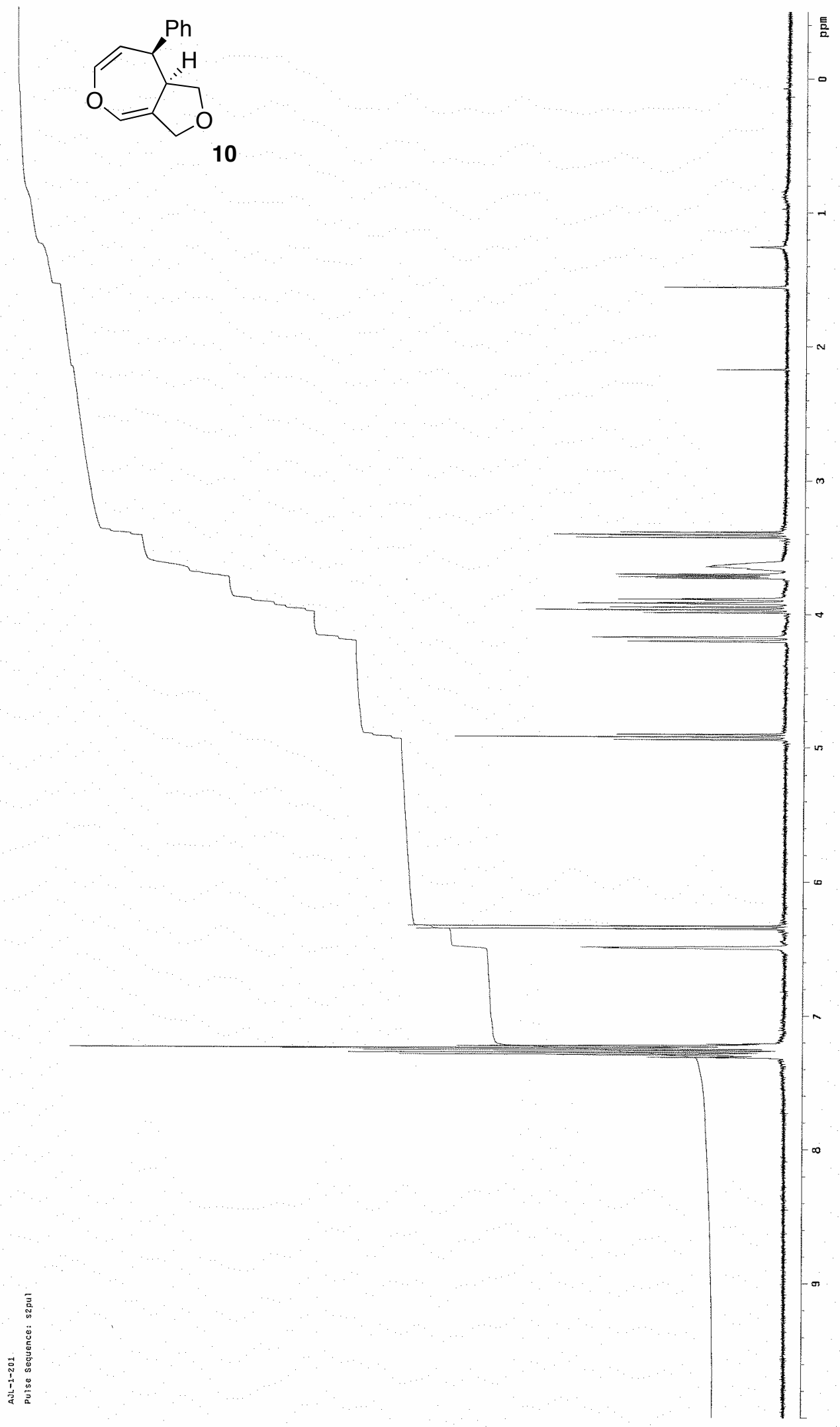




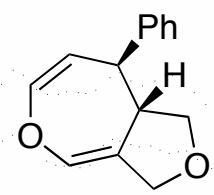

11

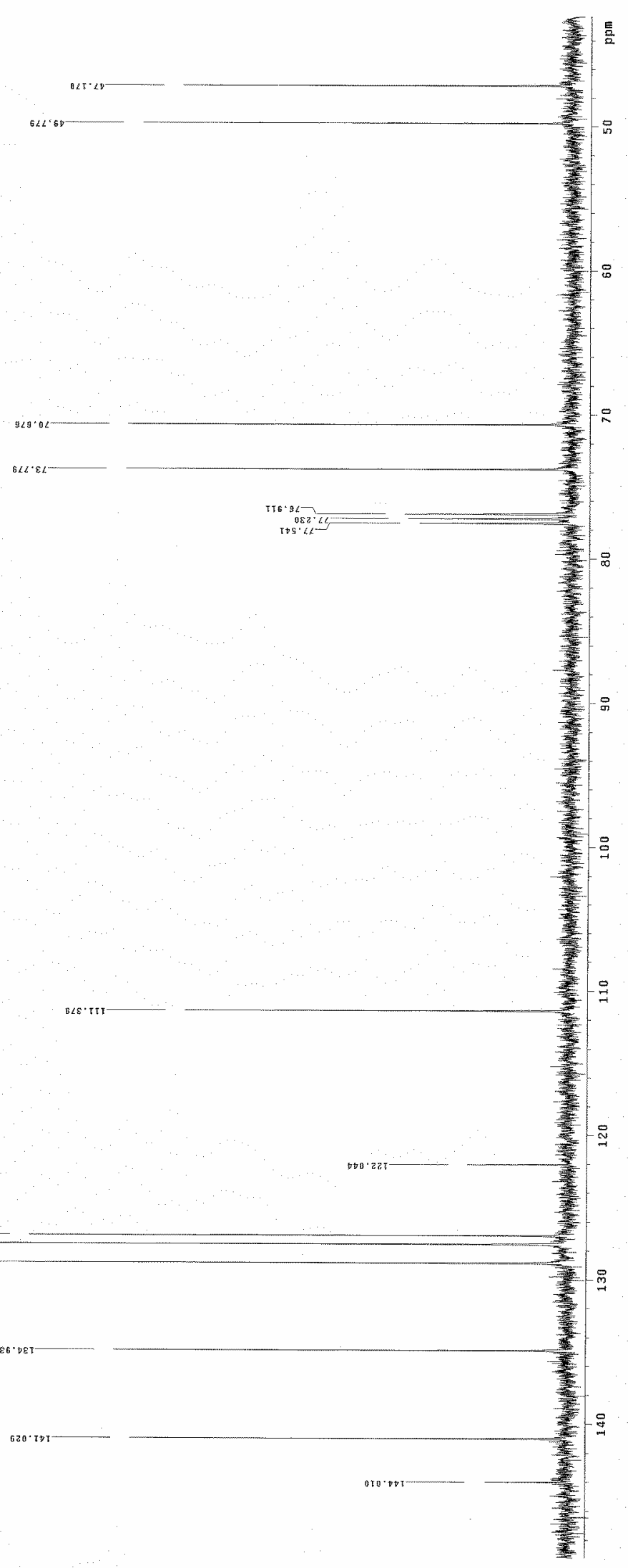




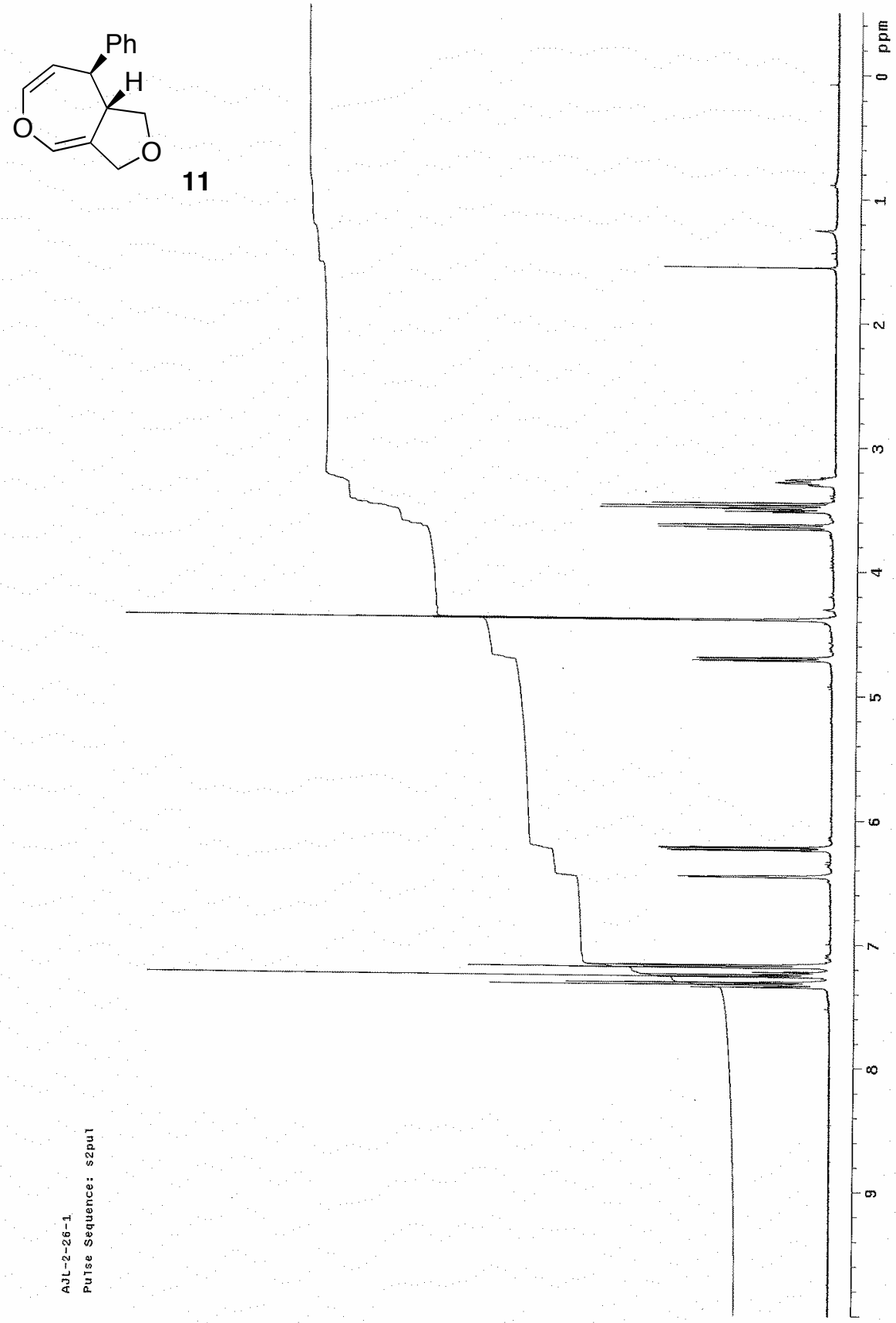




$$
\text { 列 }
$$




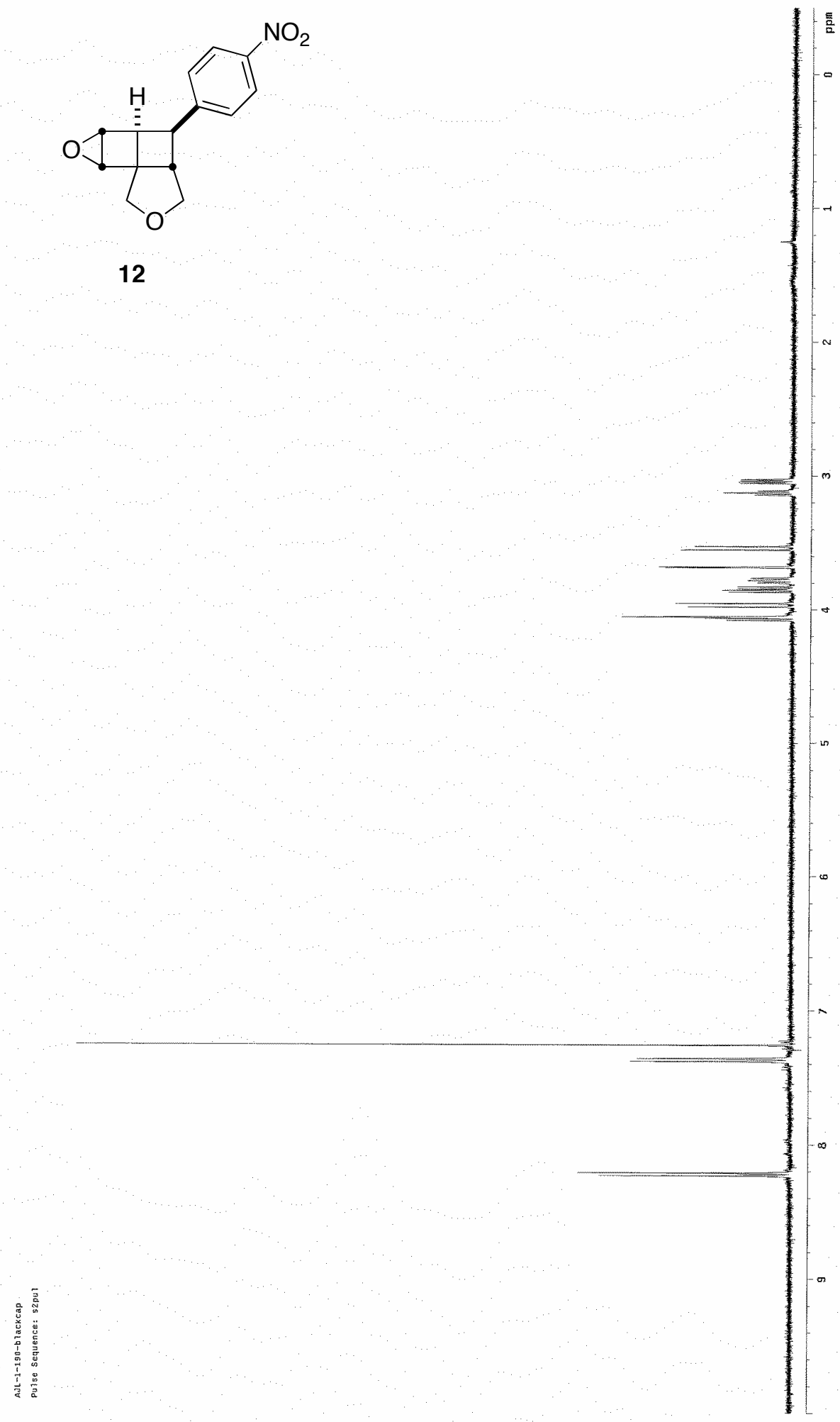



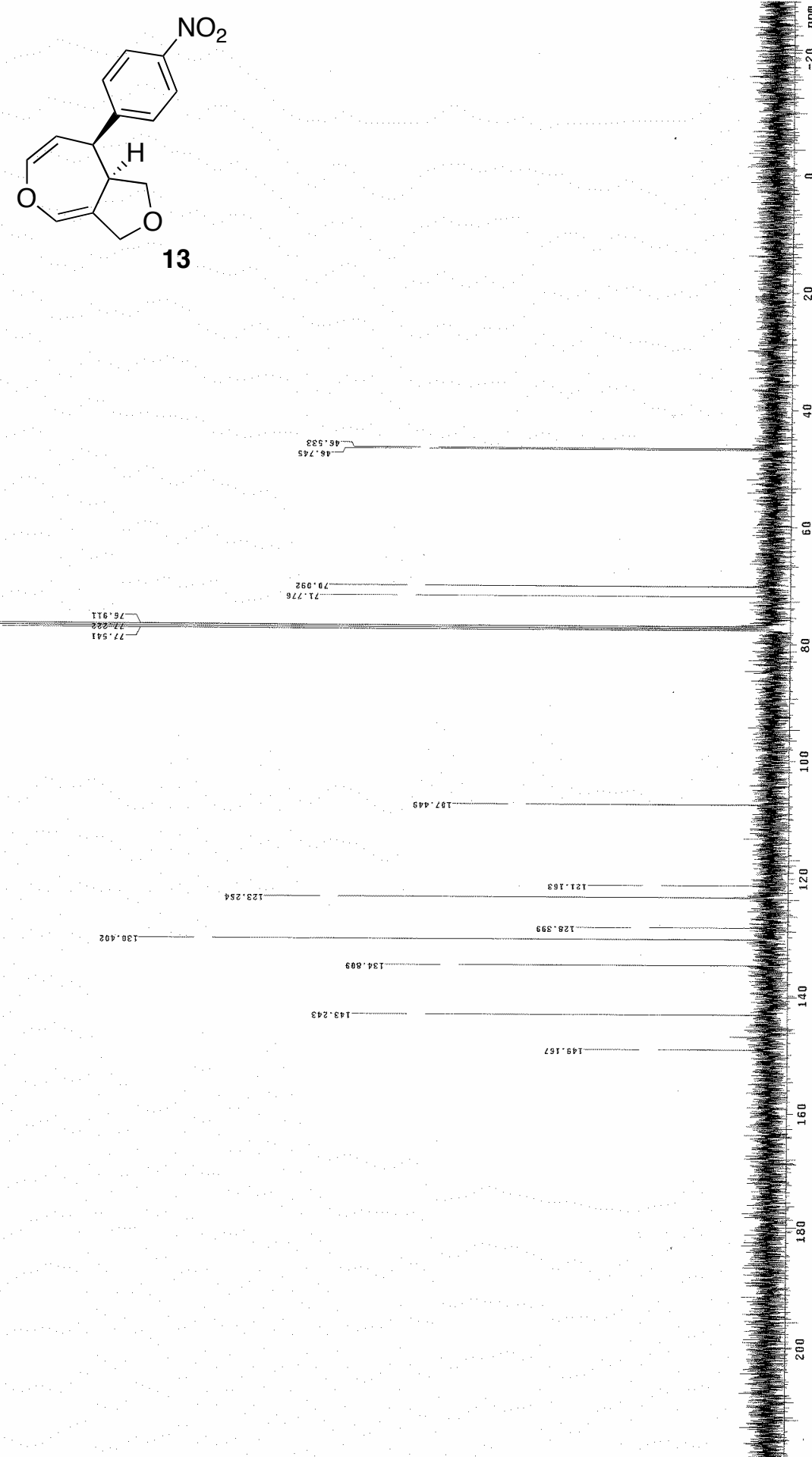

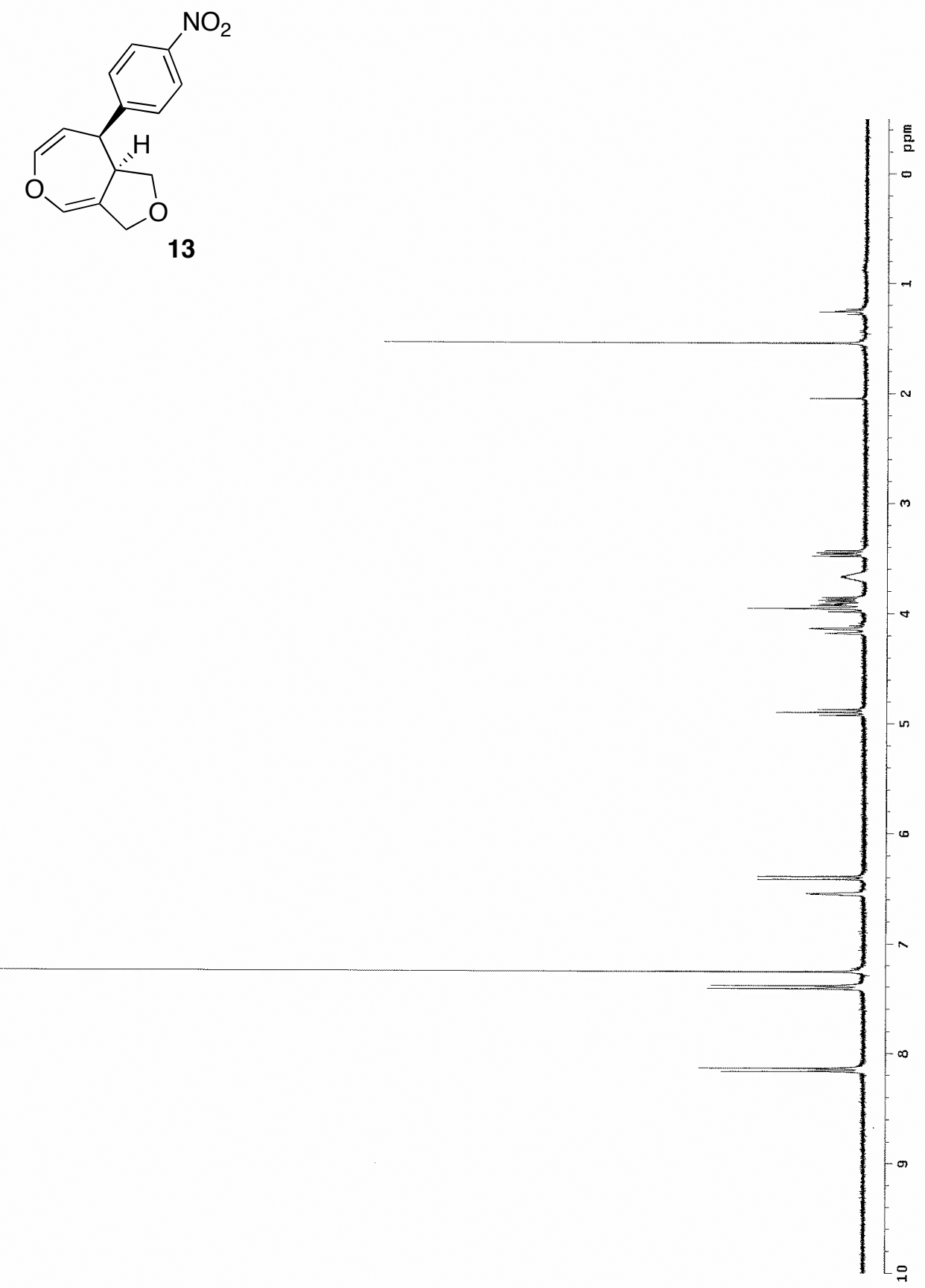
Supporting Information

SUI<smiles>O=[N+]([O-])c1ccc([C@H]2C=COC=C3COC[C@H]32)cc1</smiles>

14

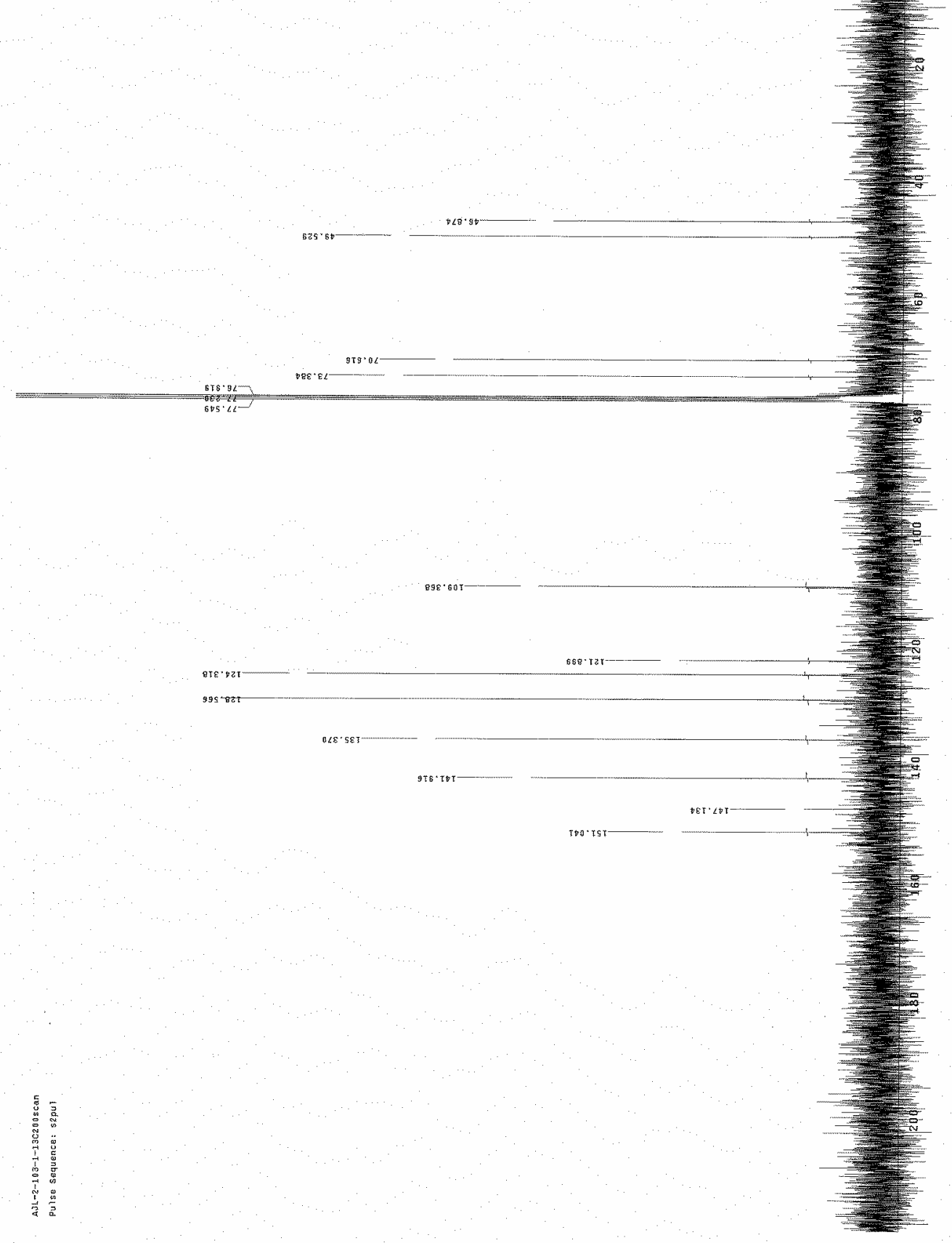



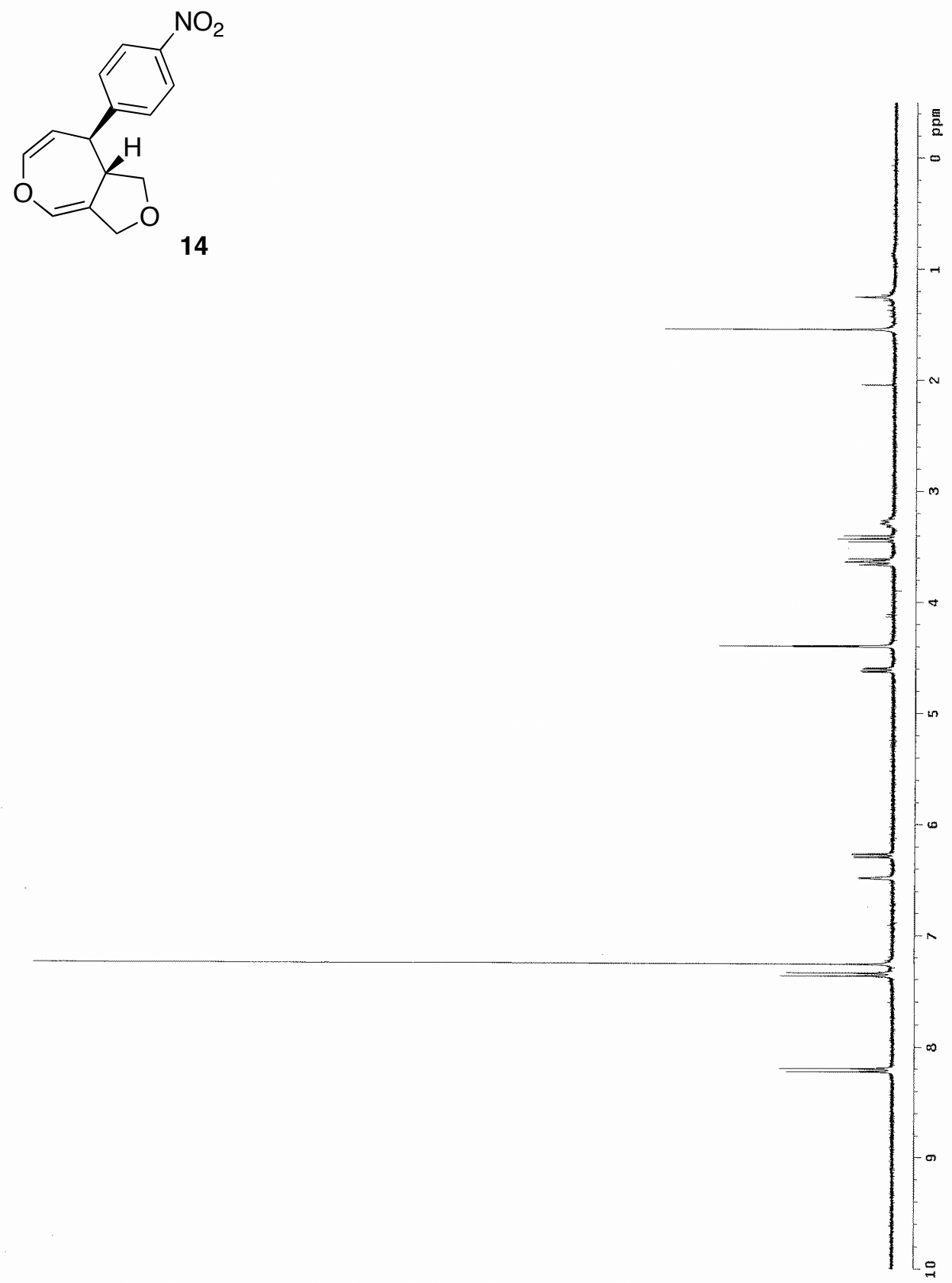


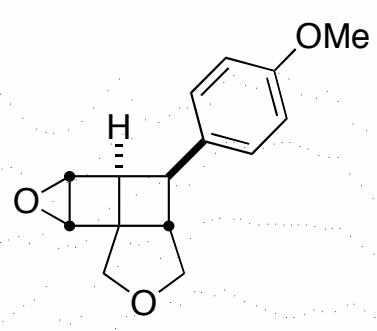

15

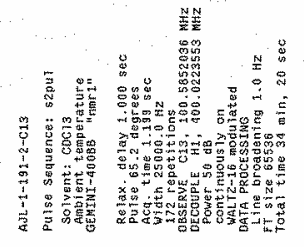




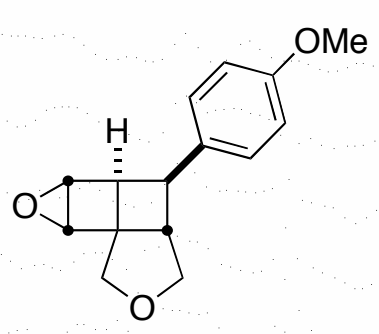

15
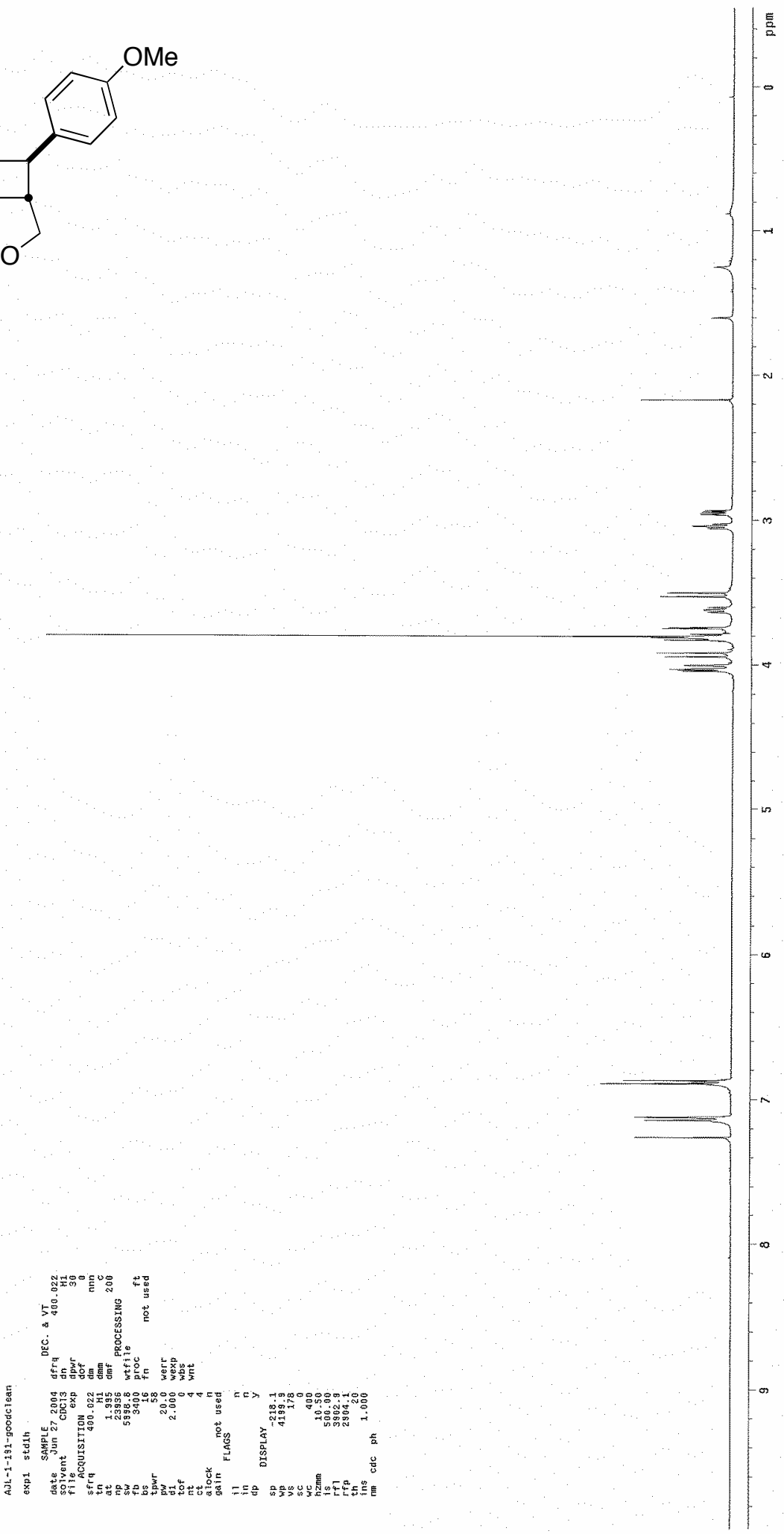

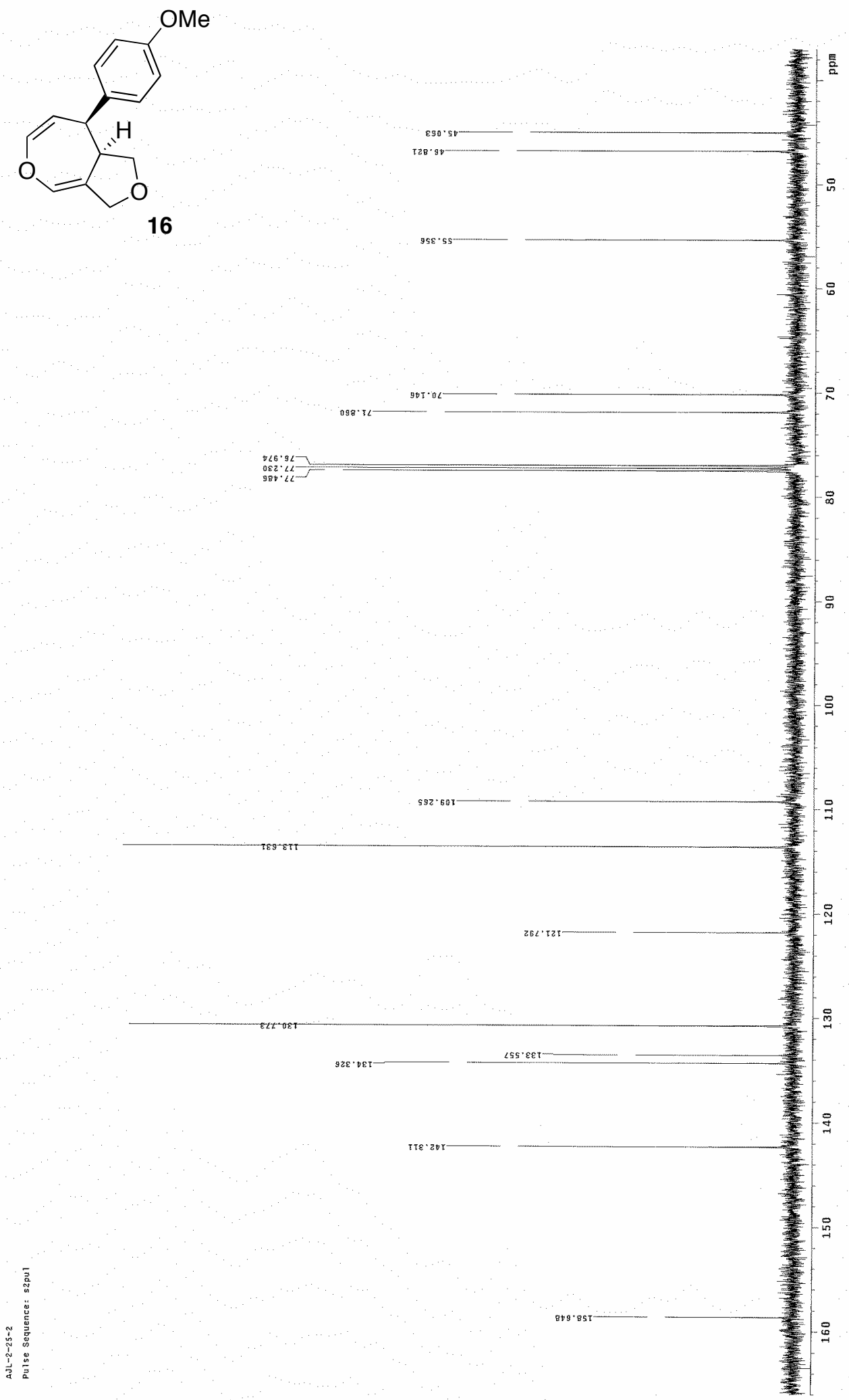


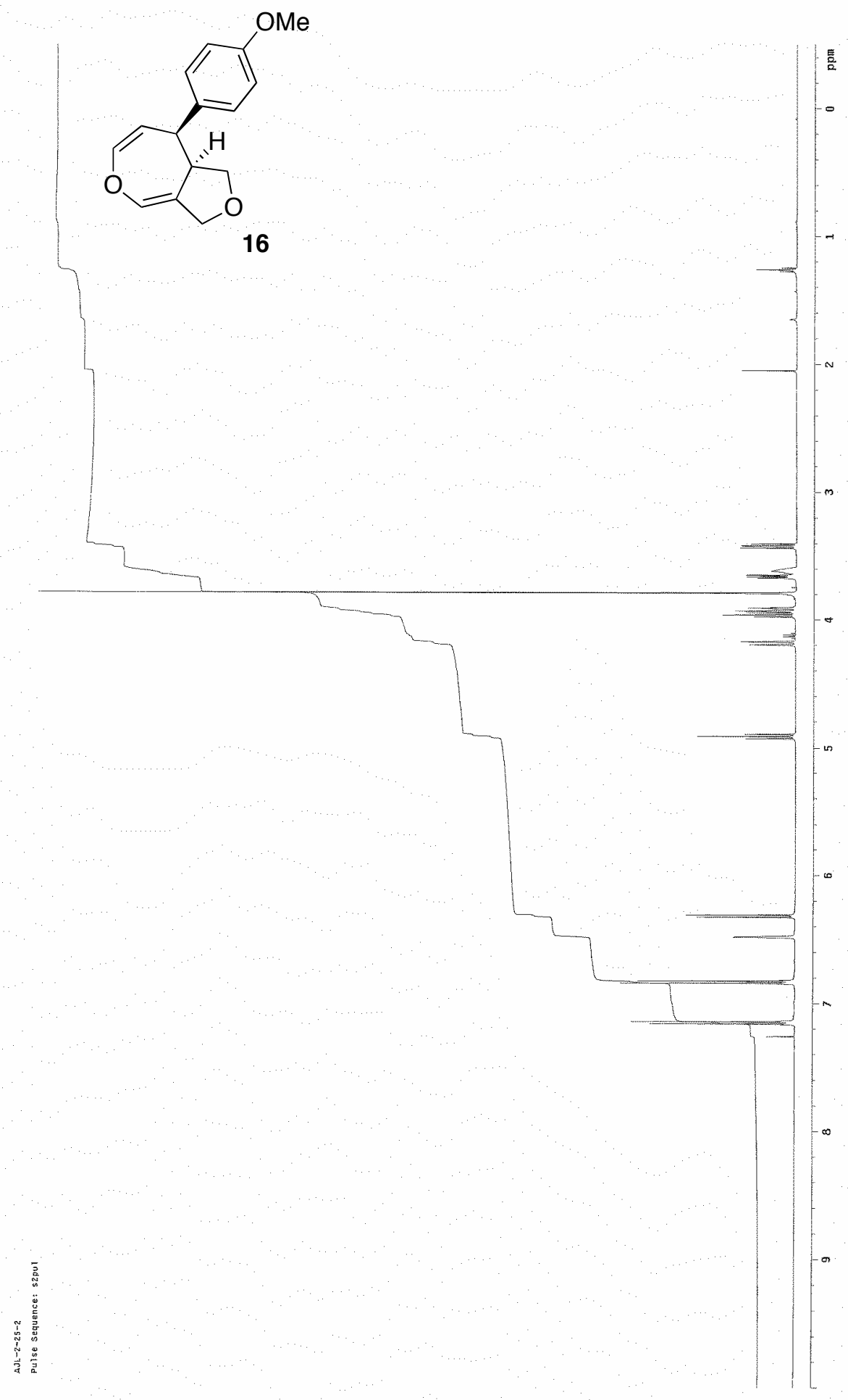



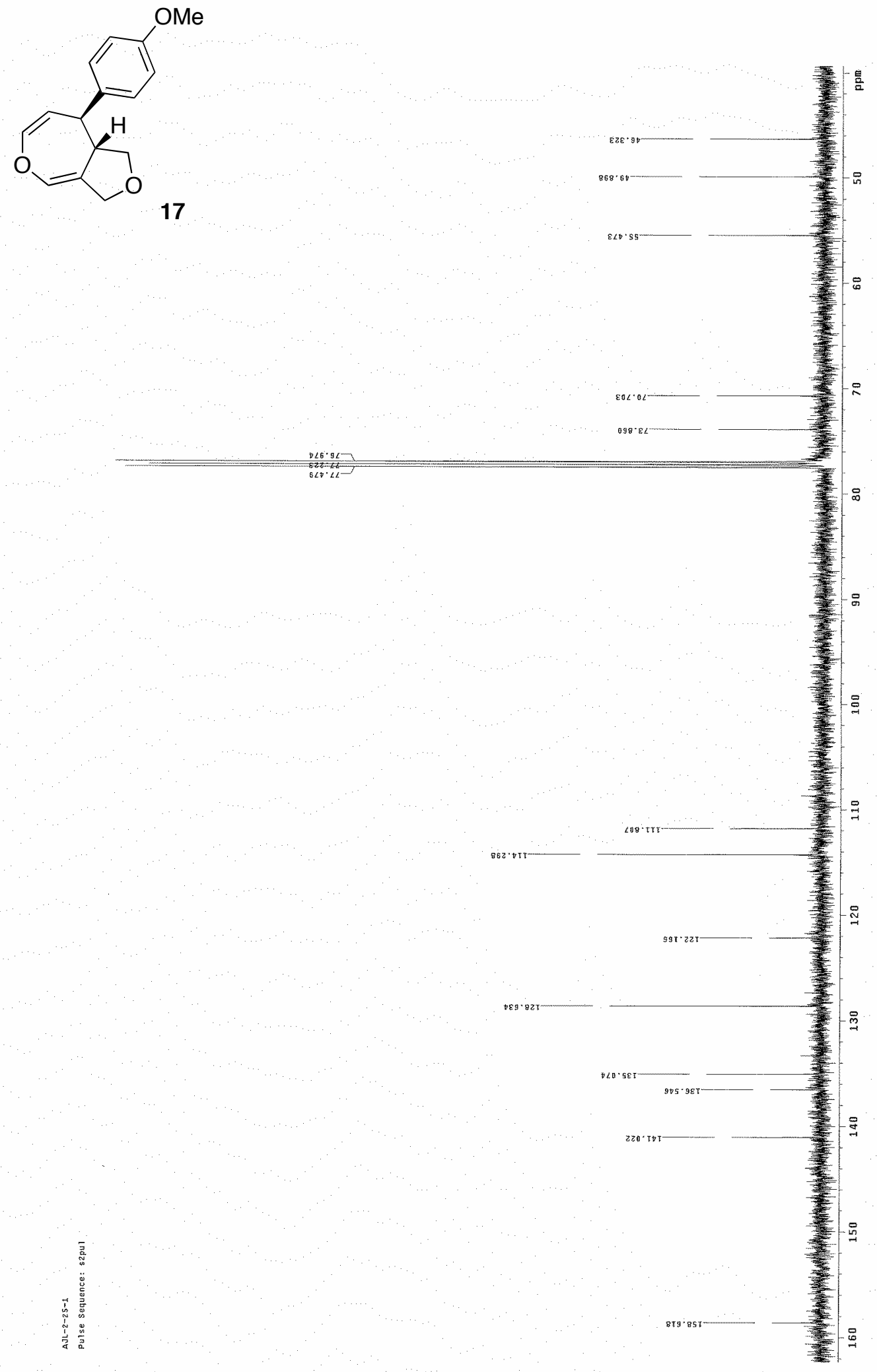


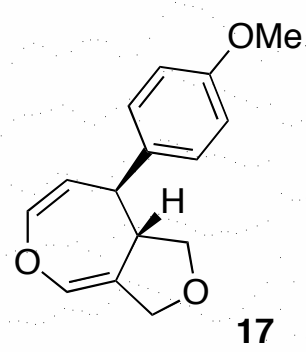

17
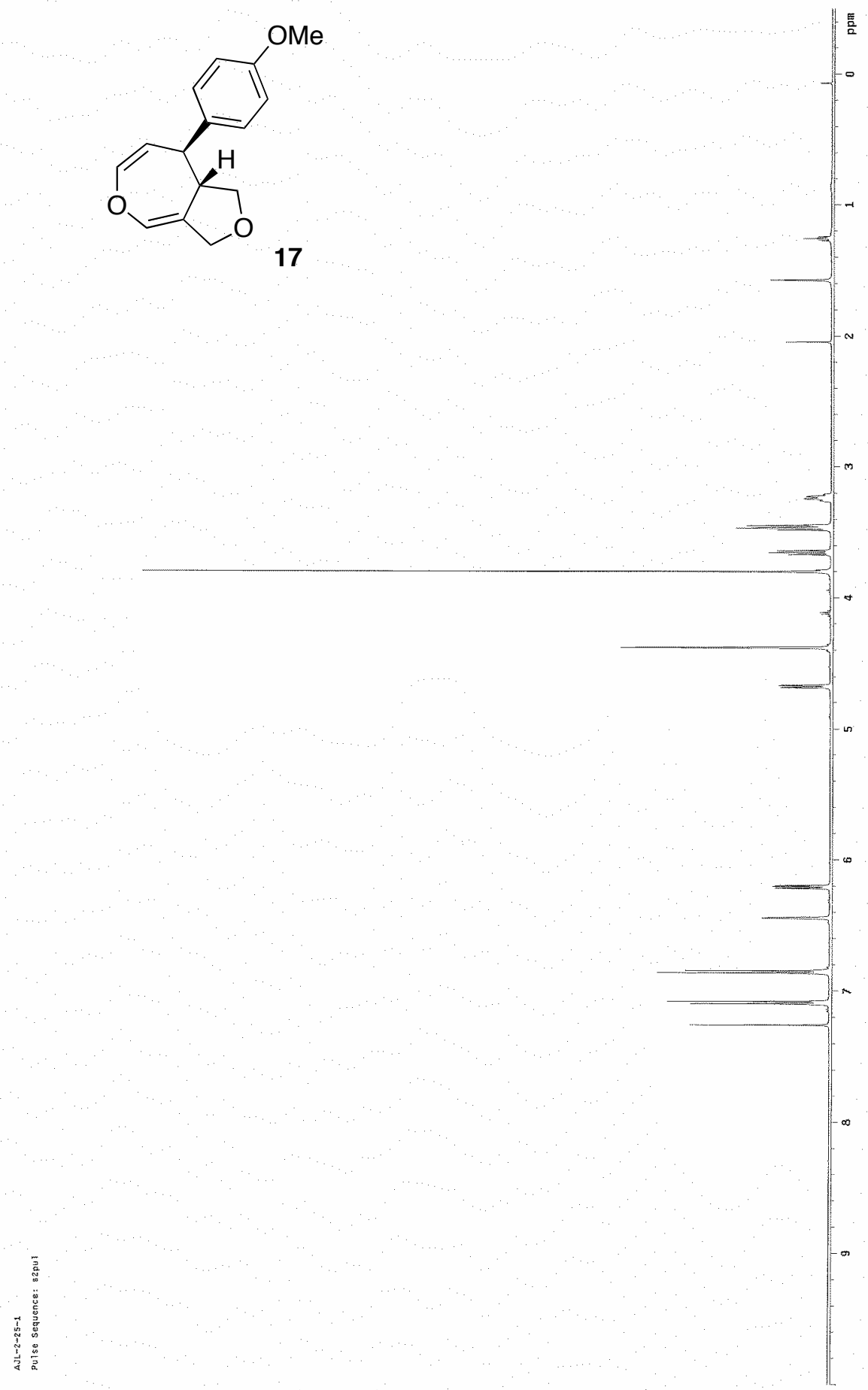

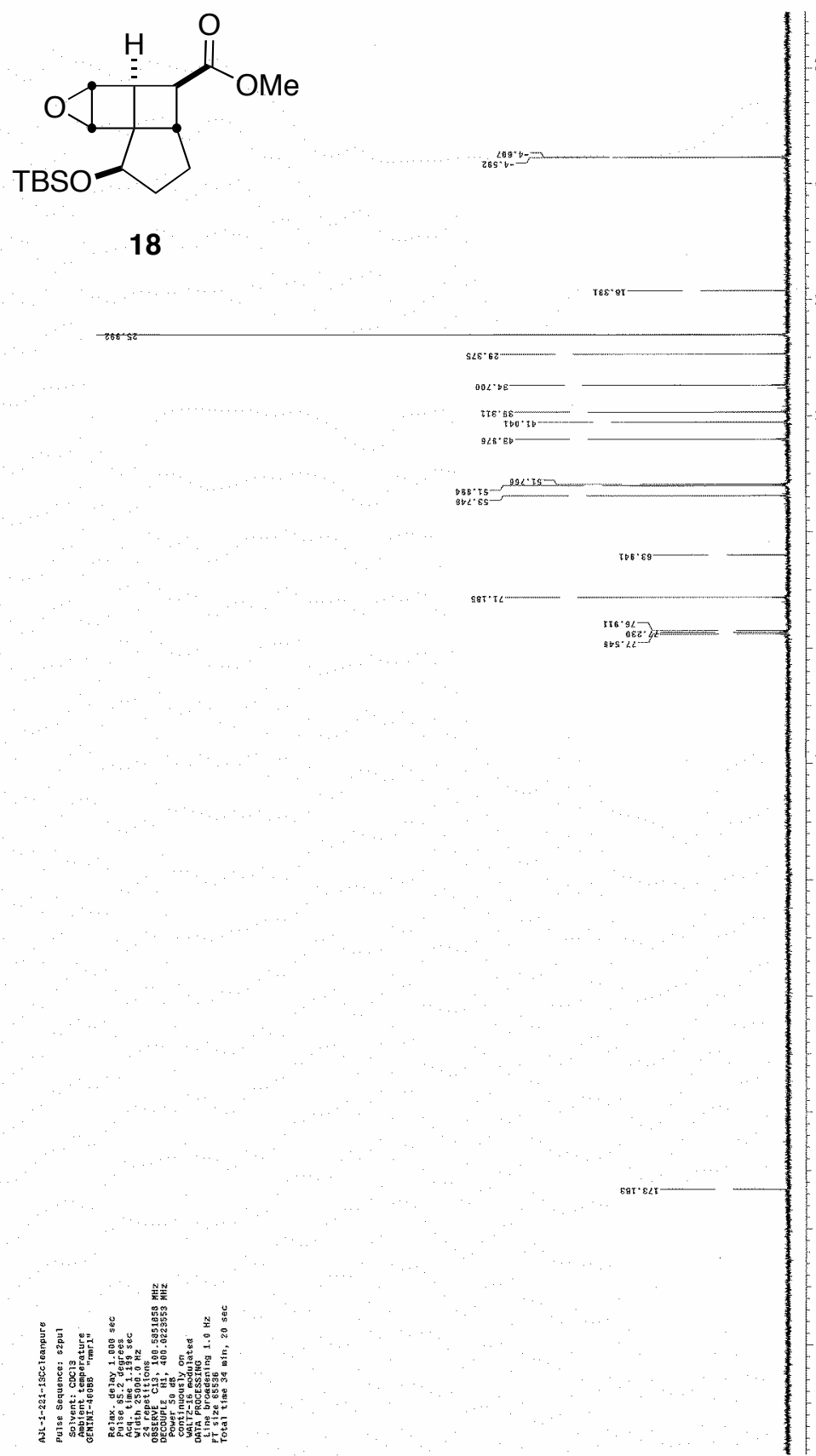


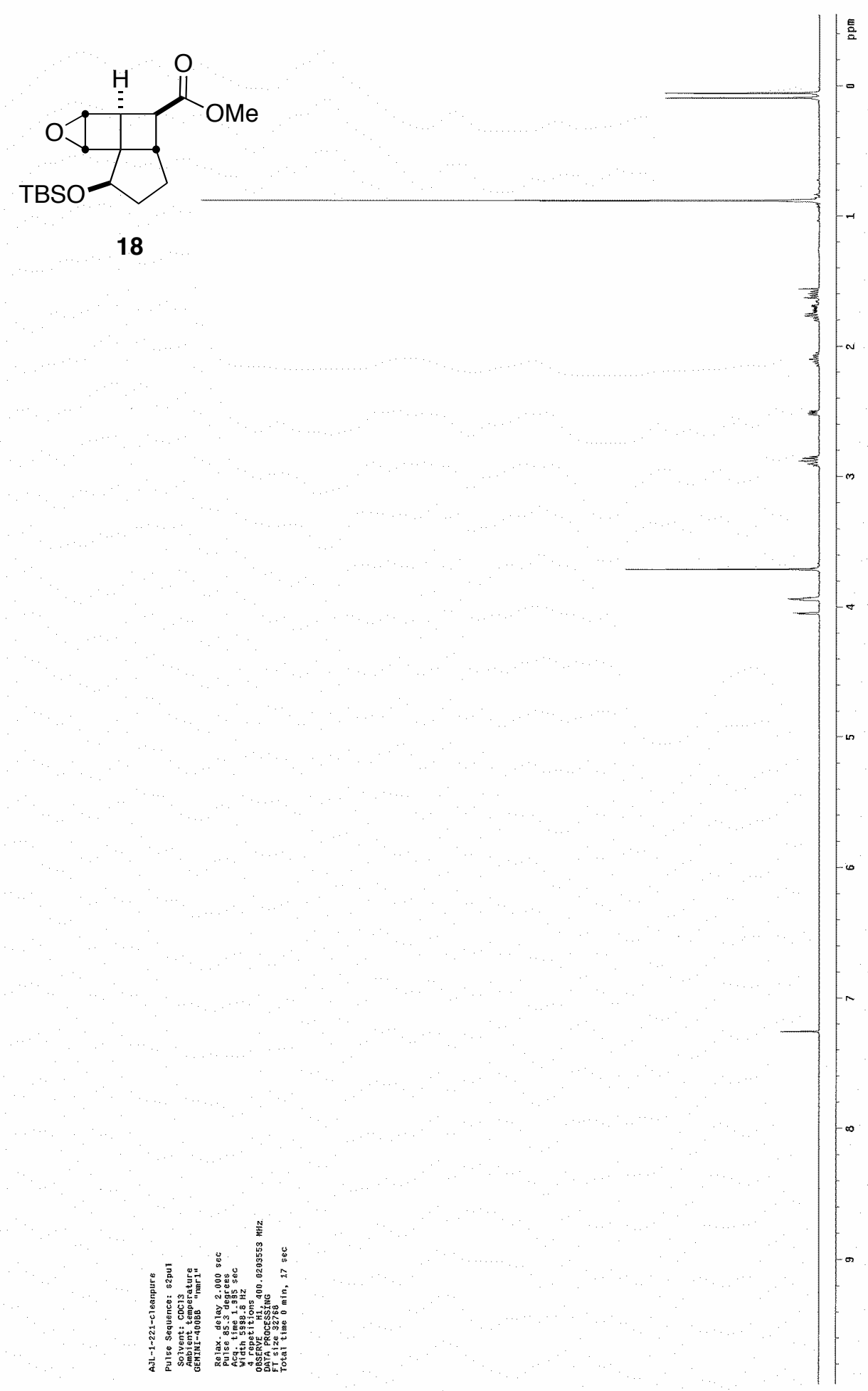




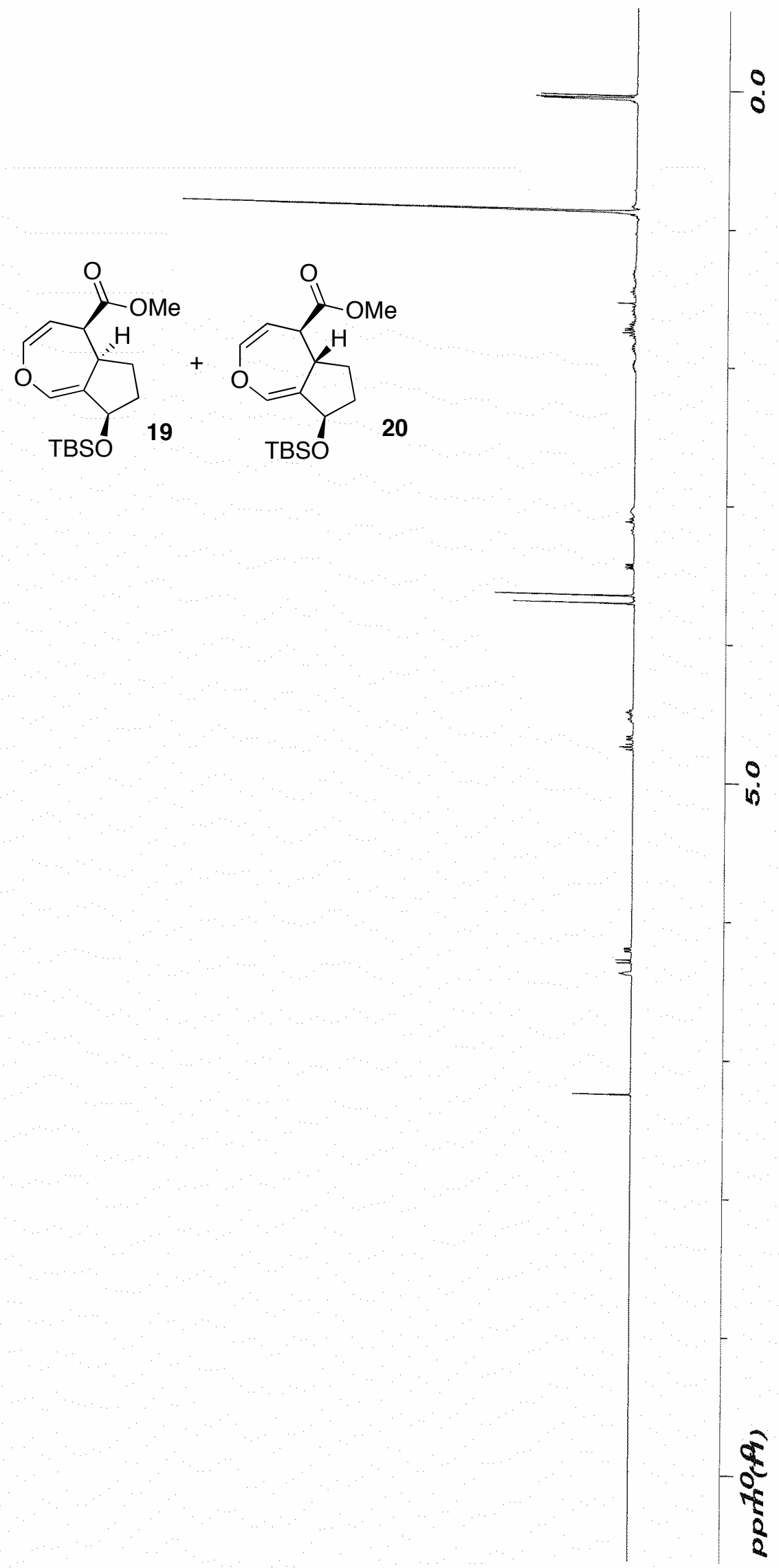




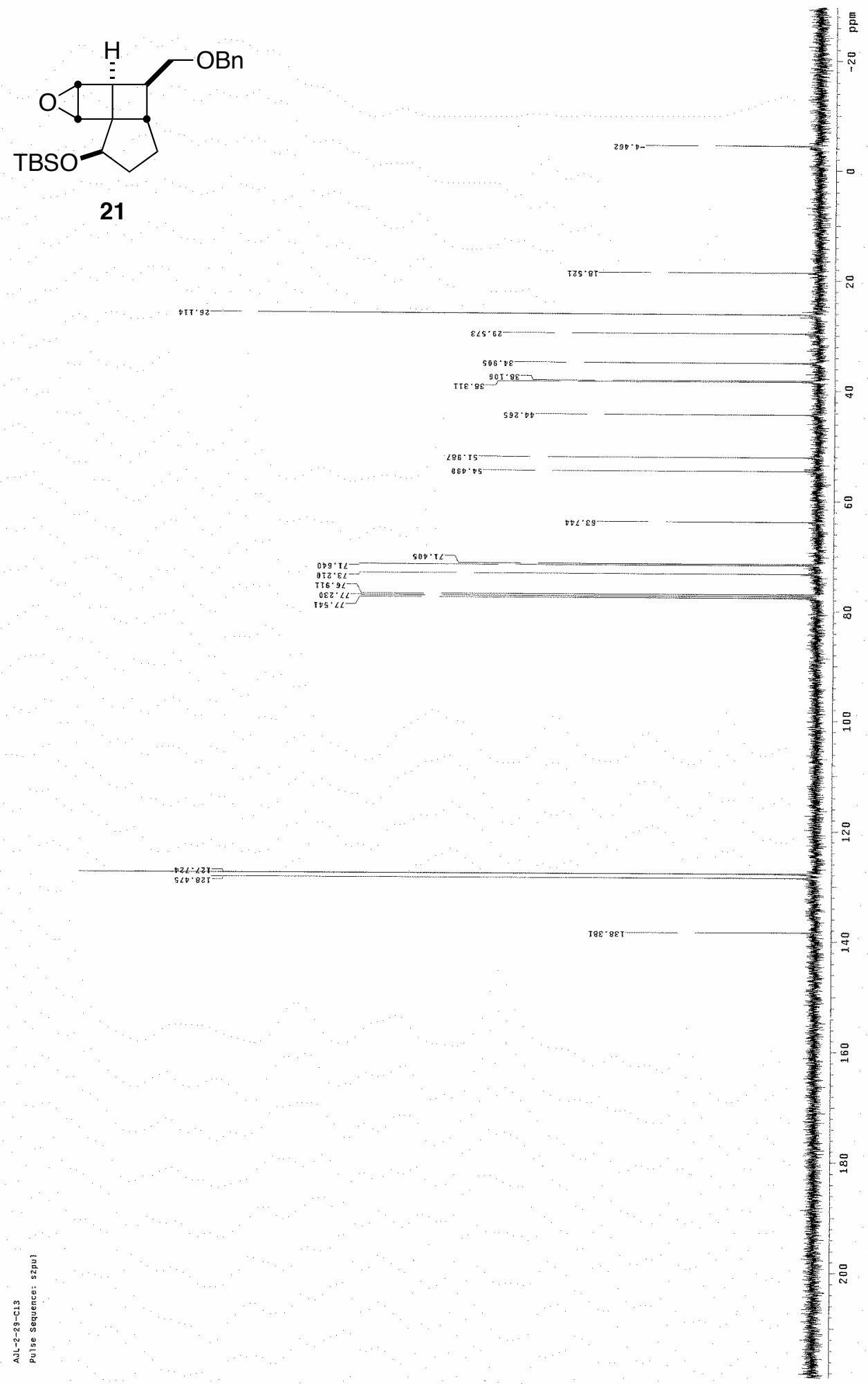




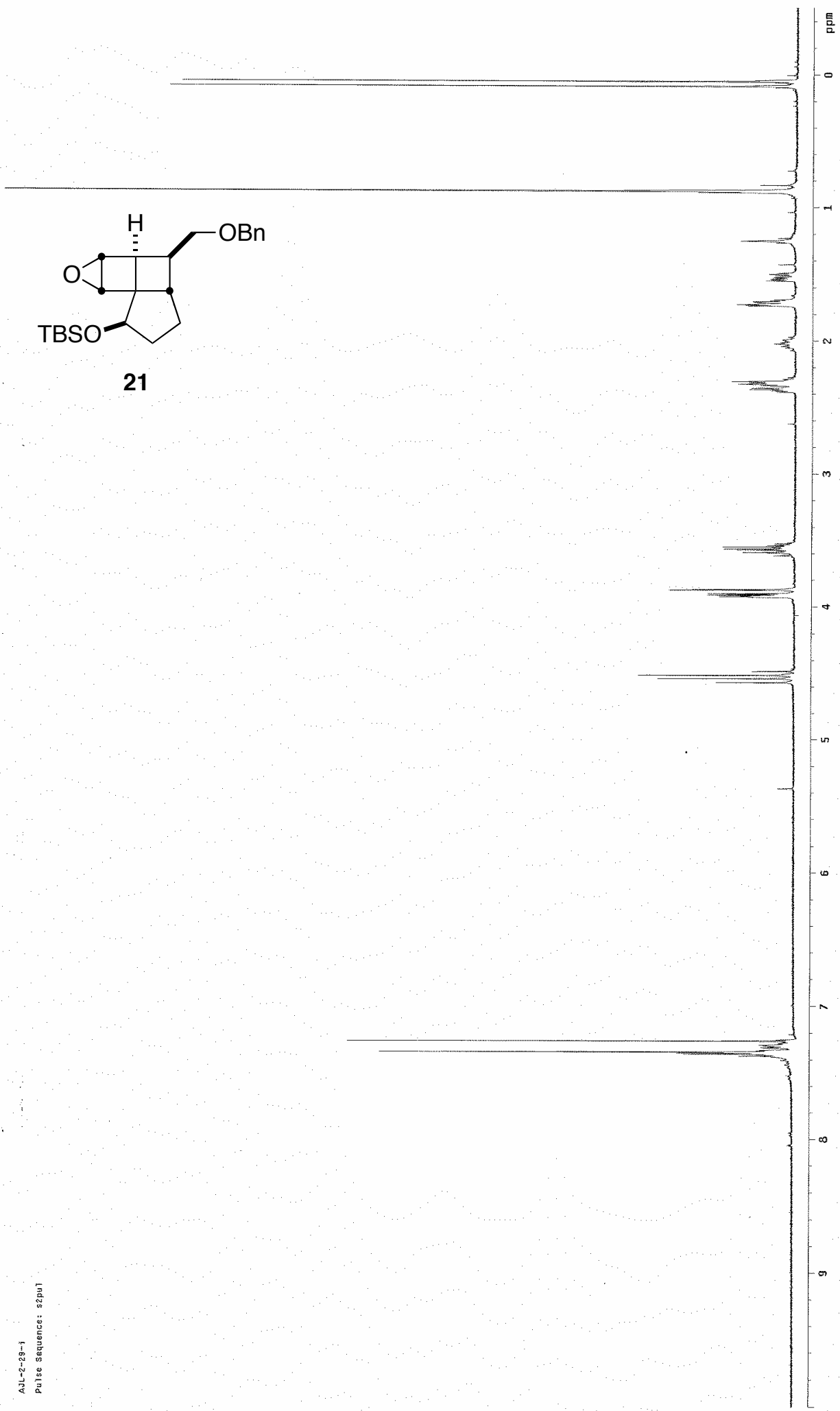




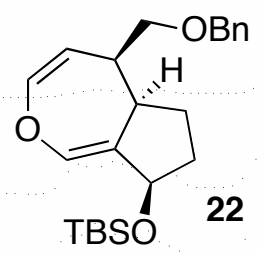

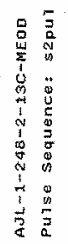

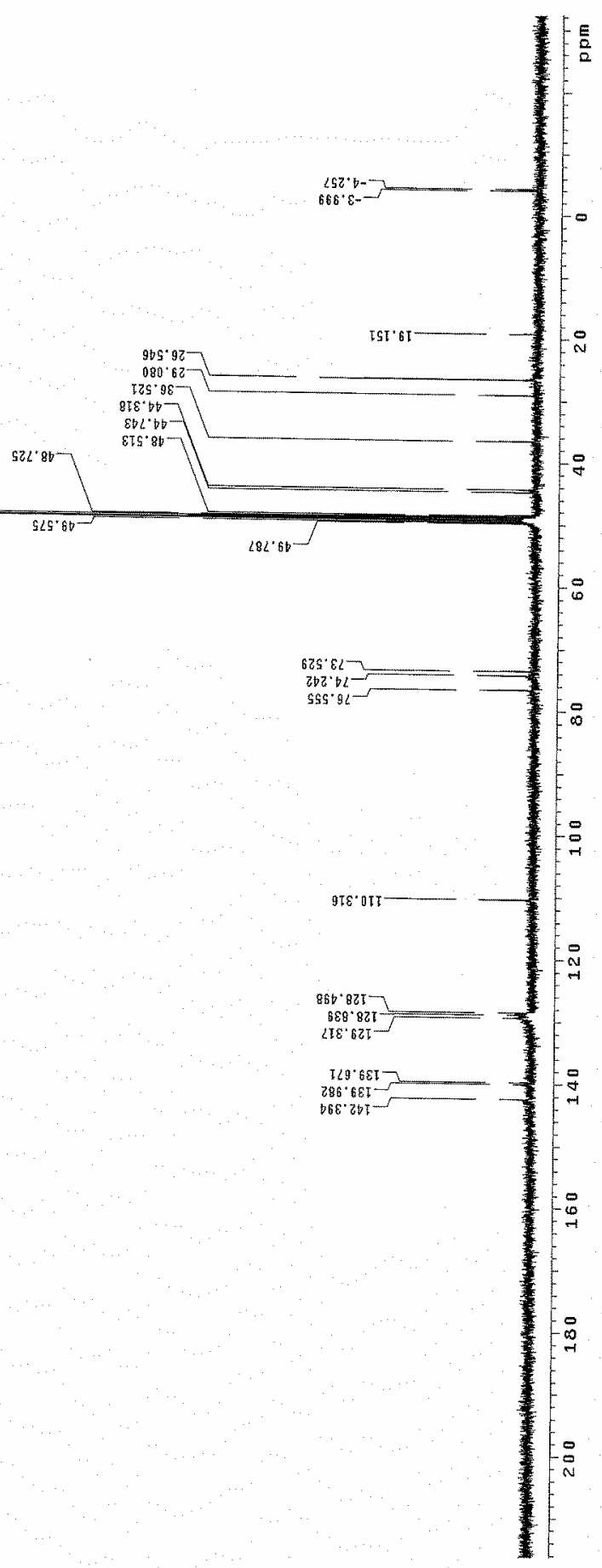




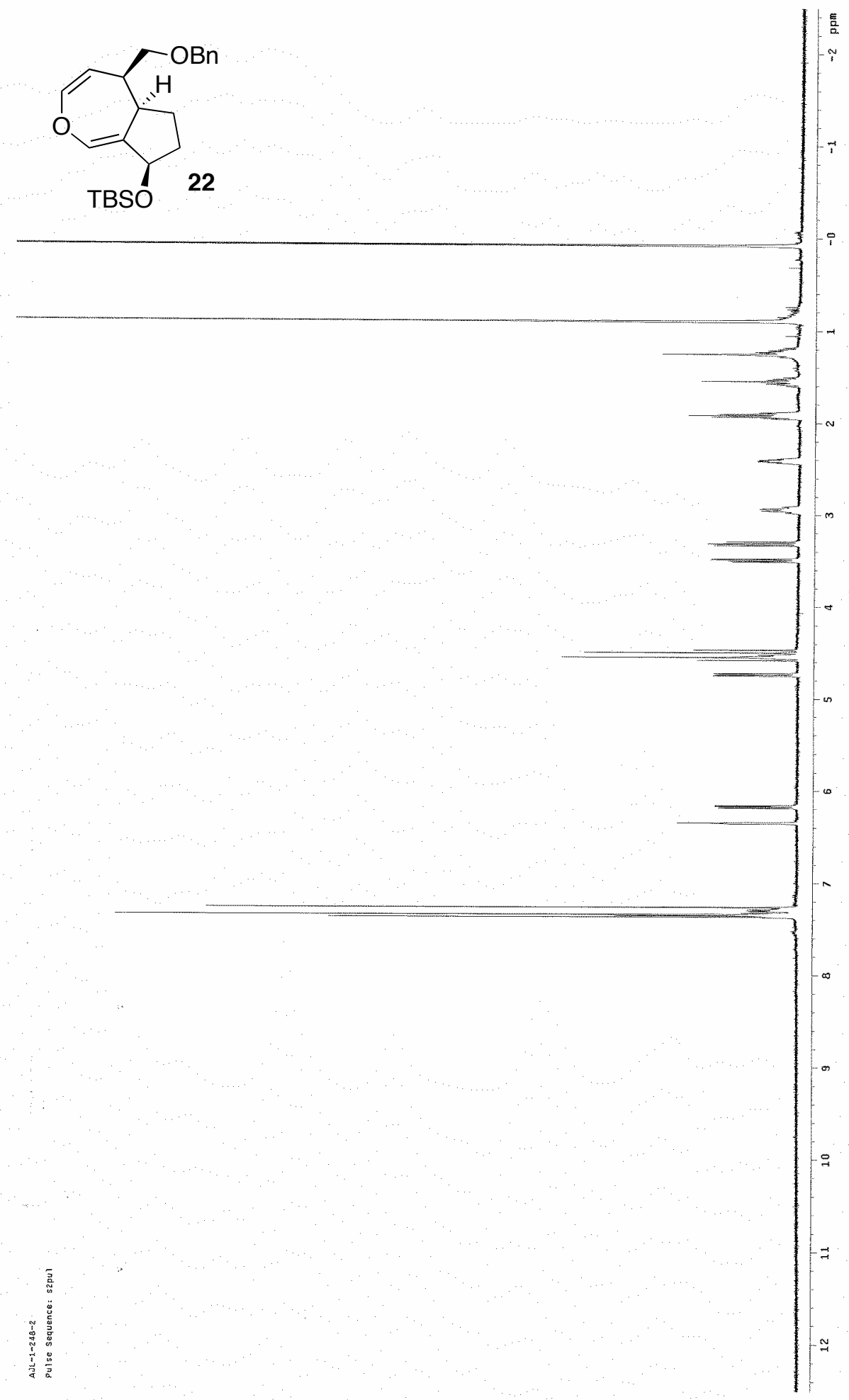




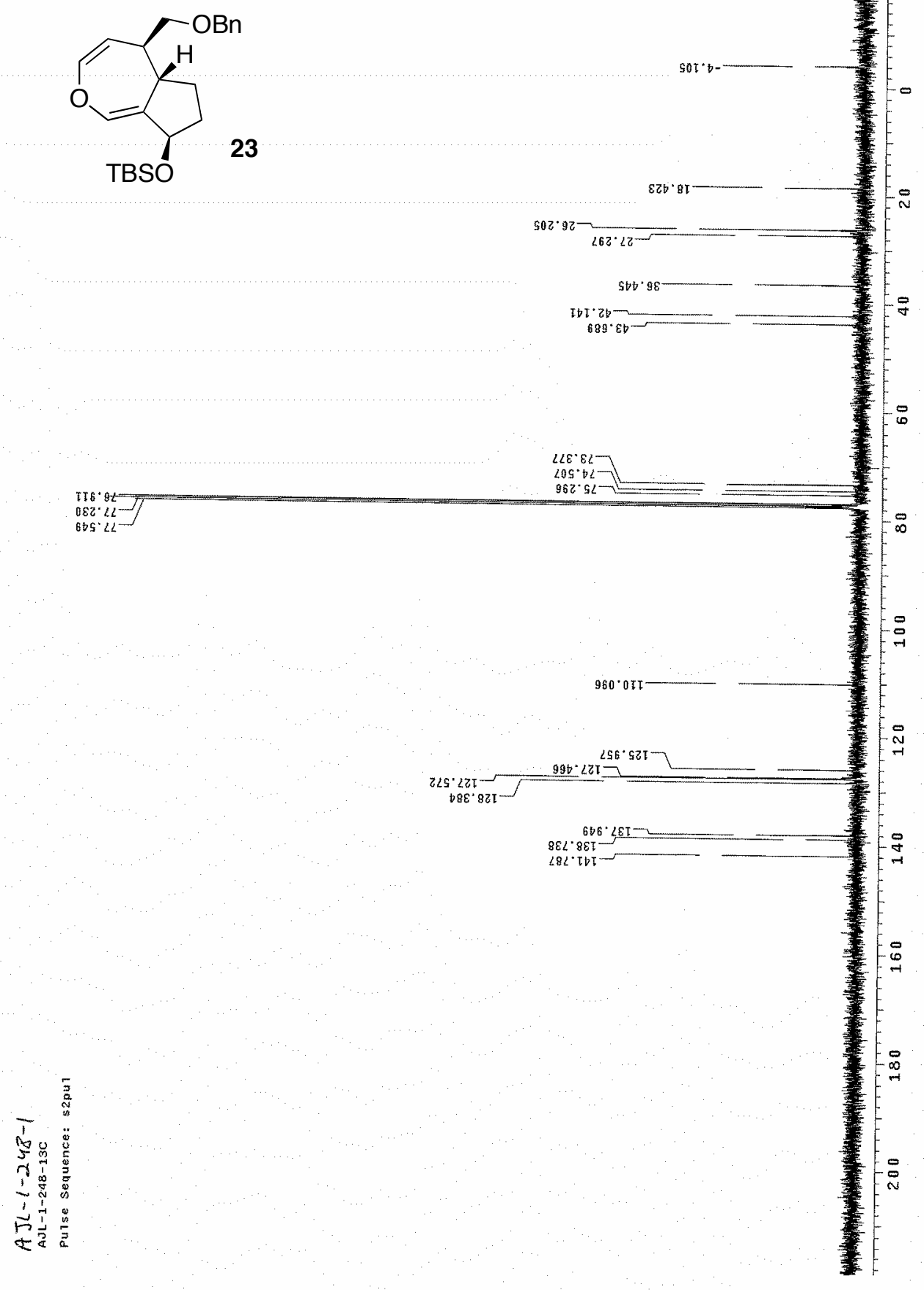




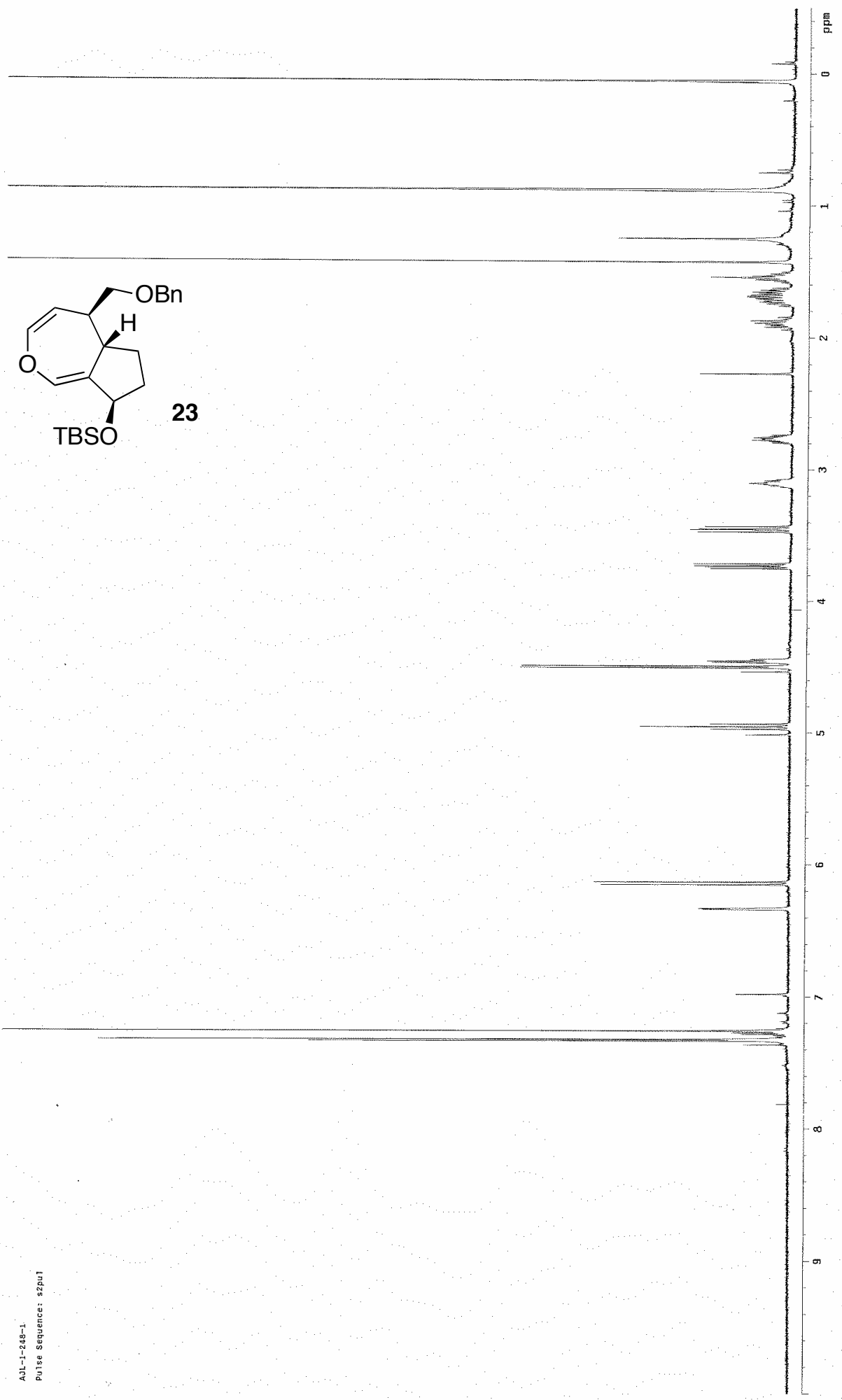



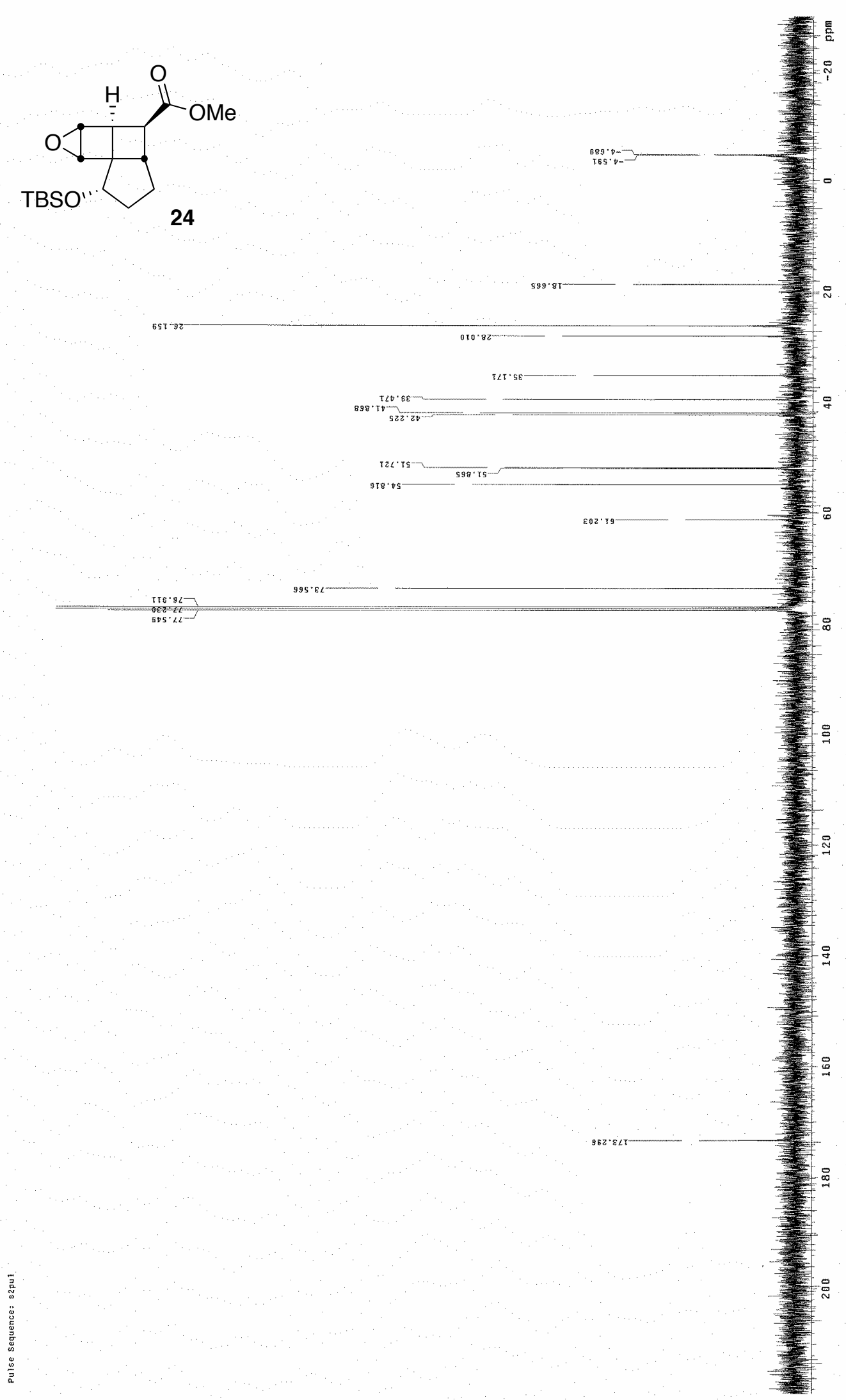


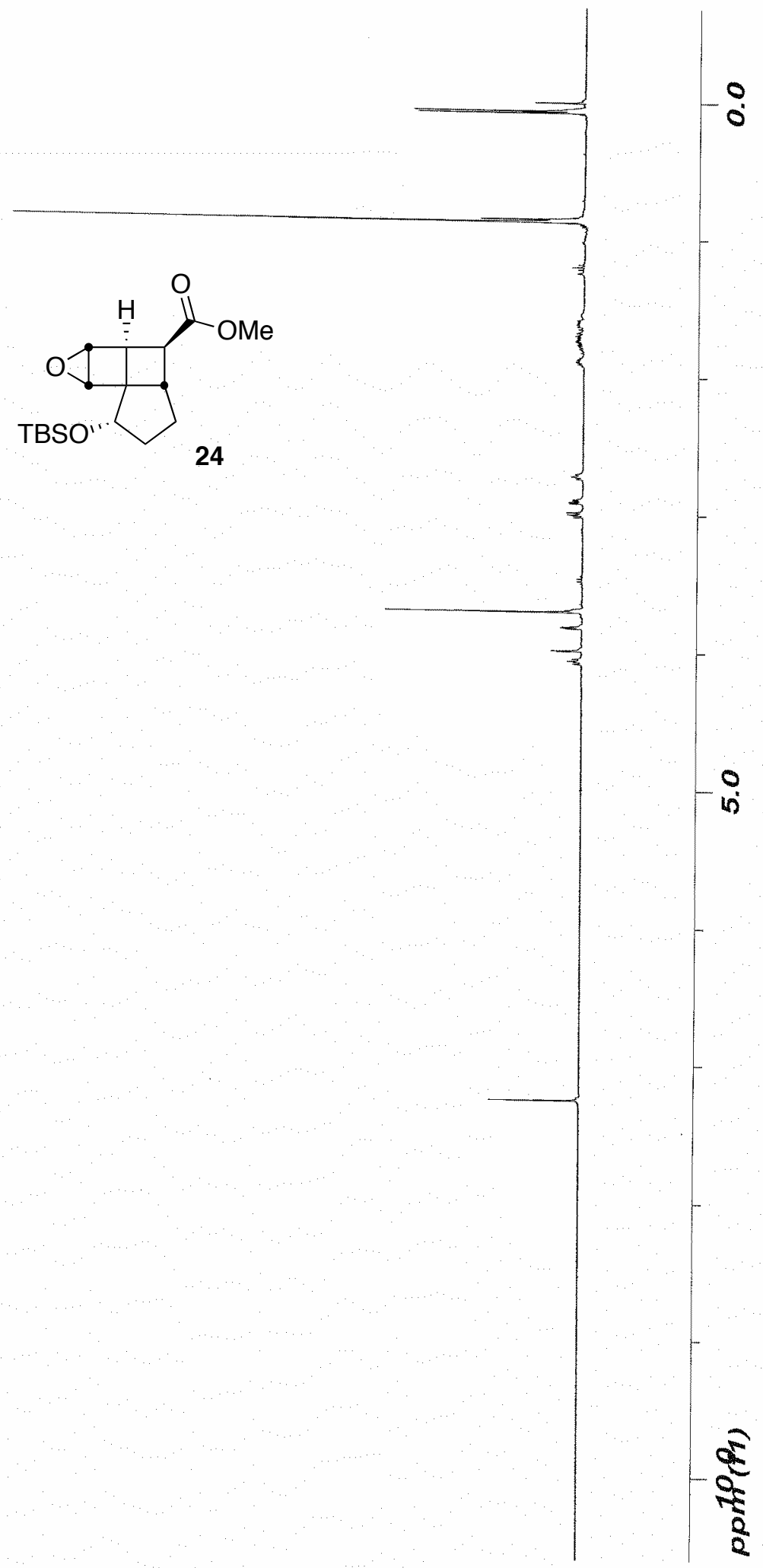




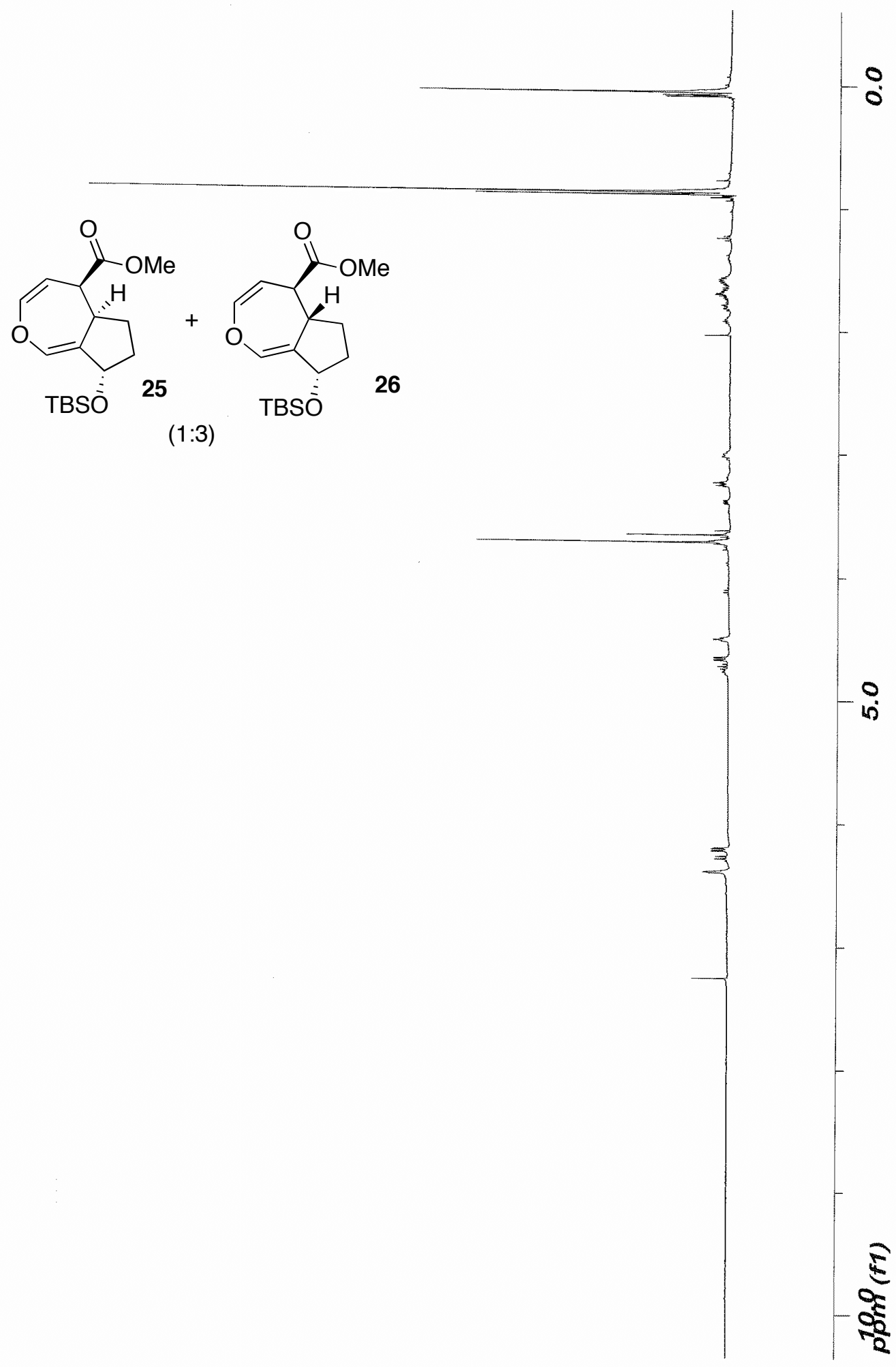



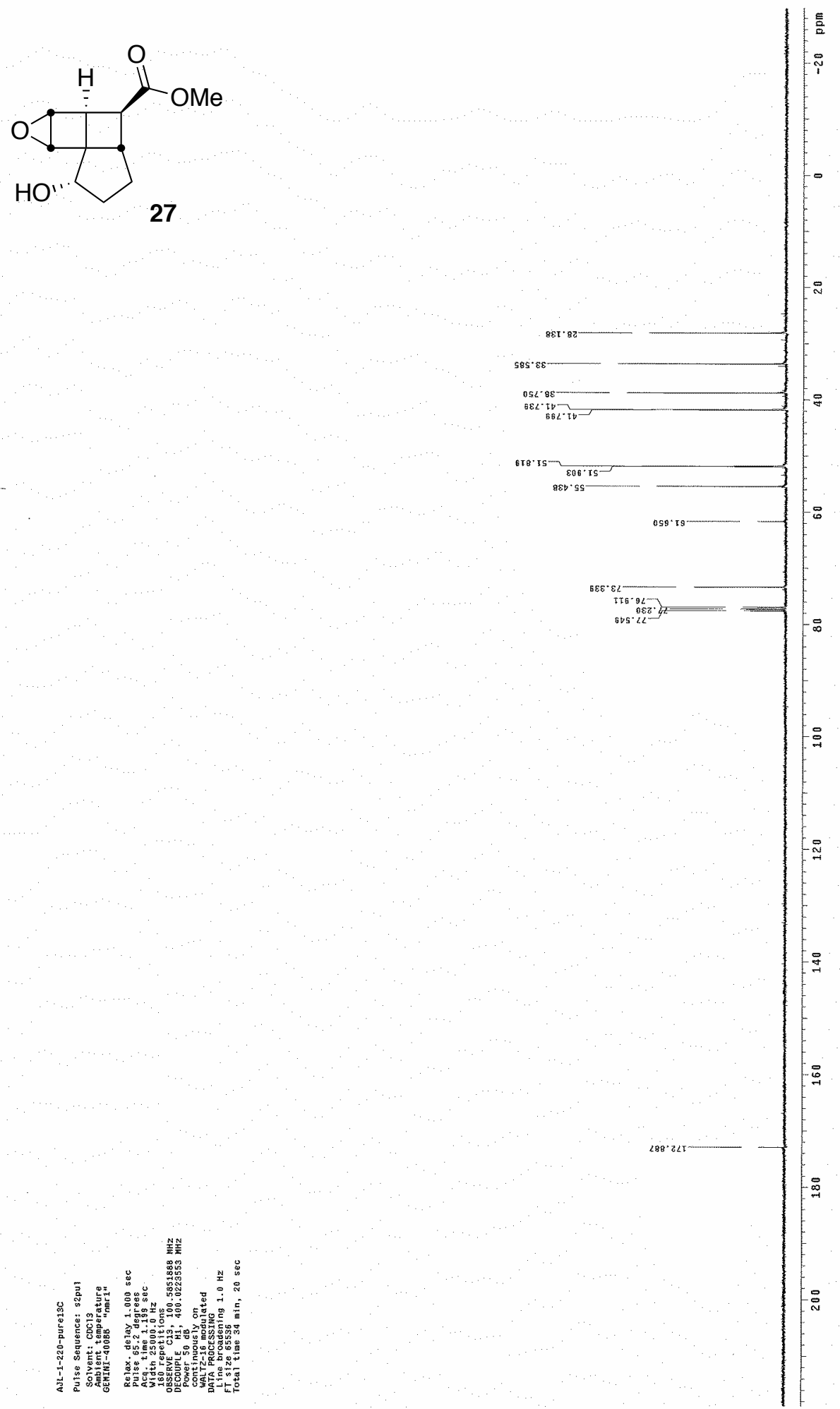

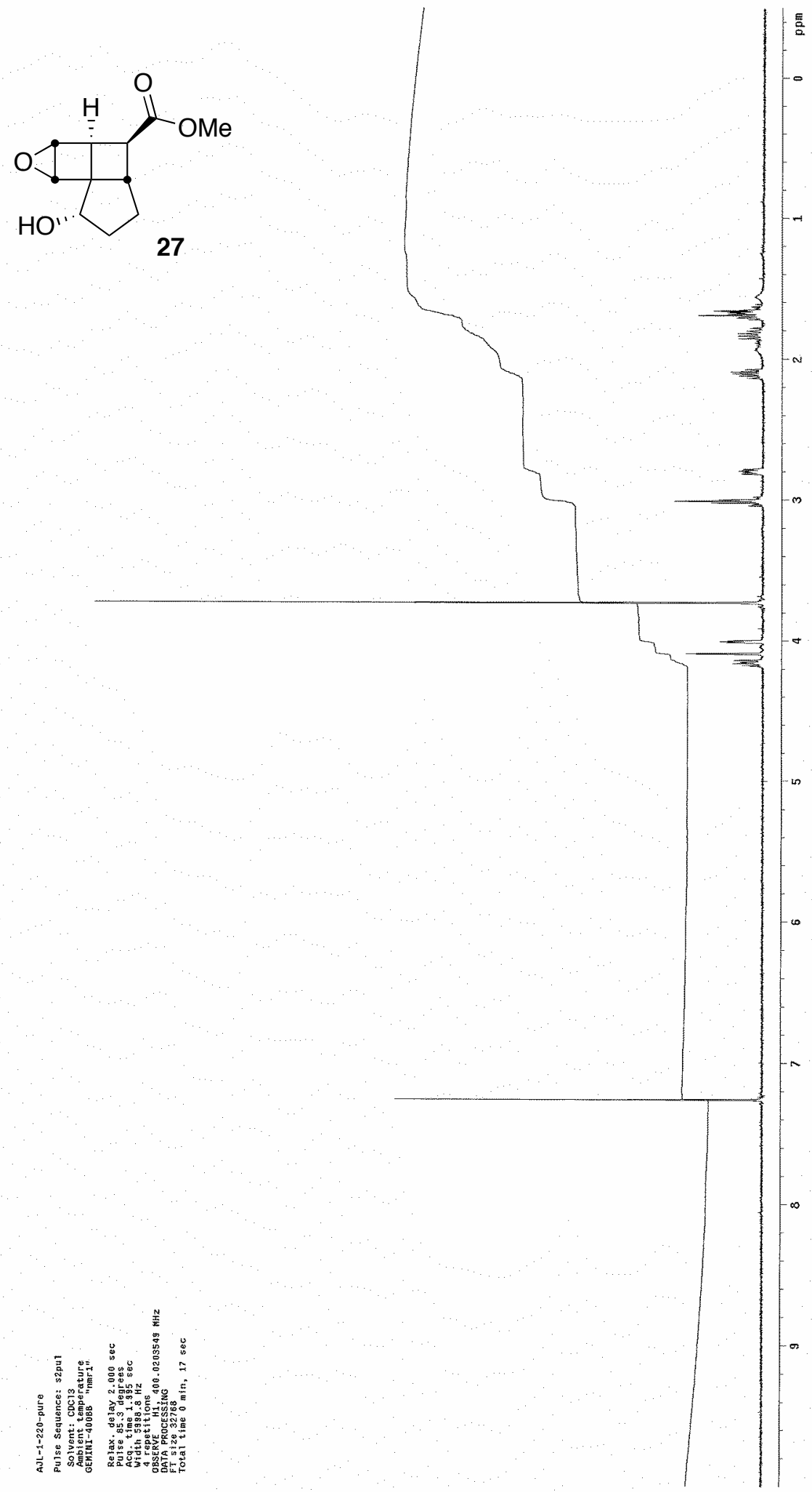

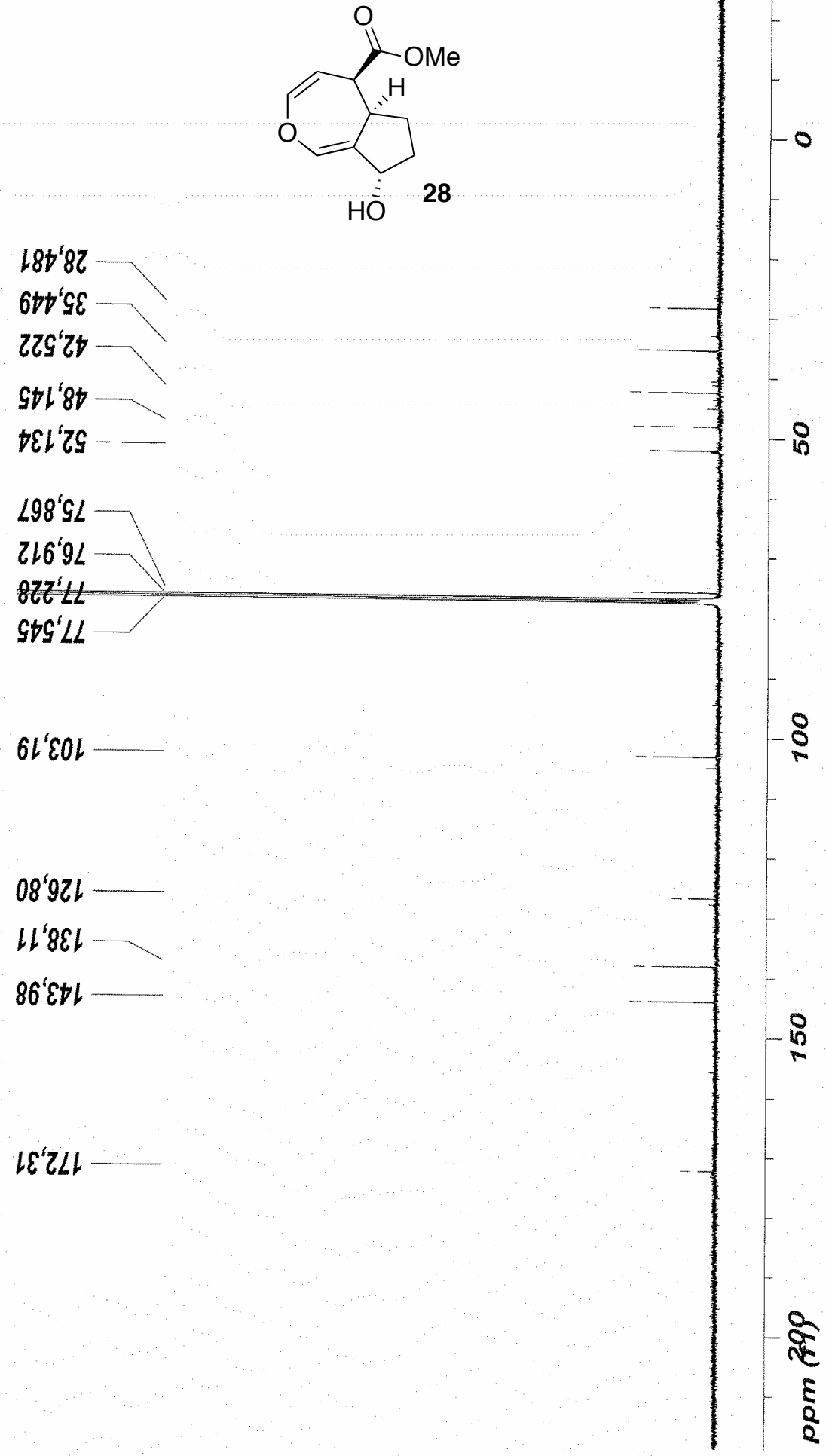


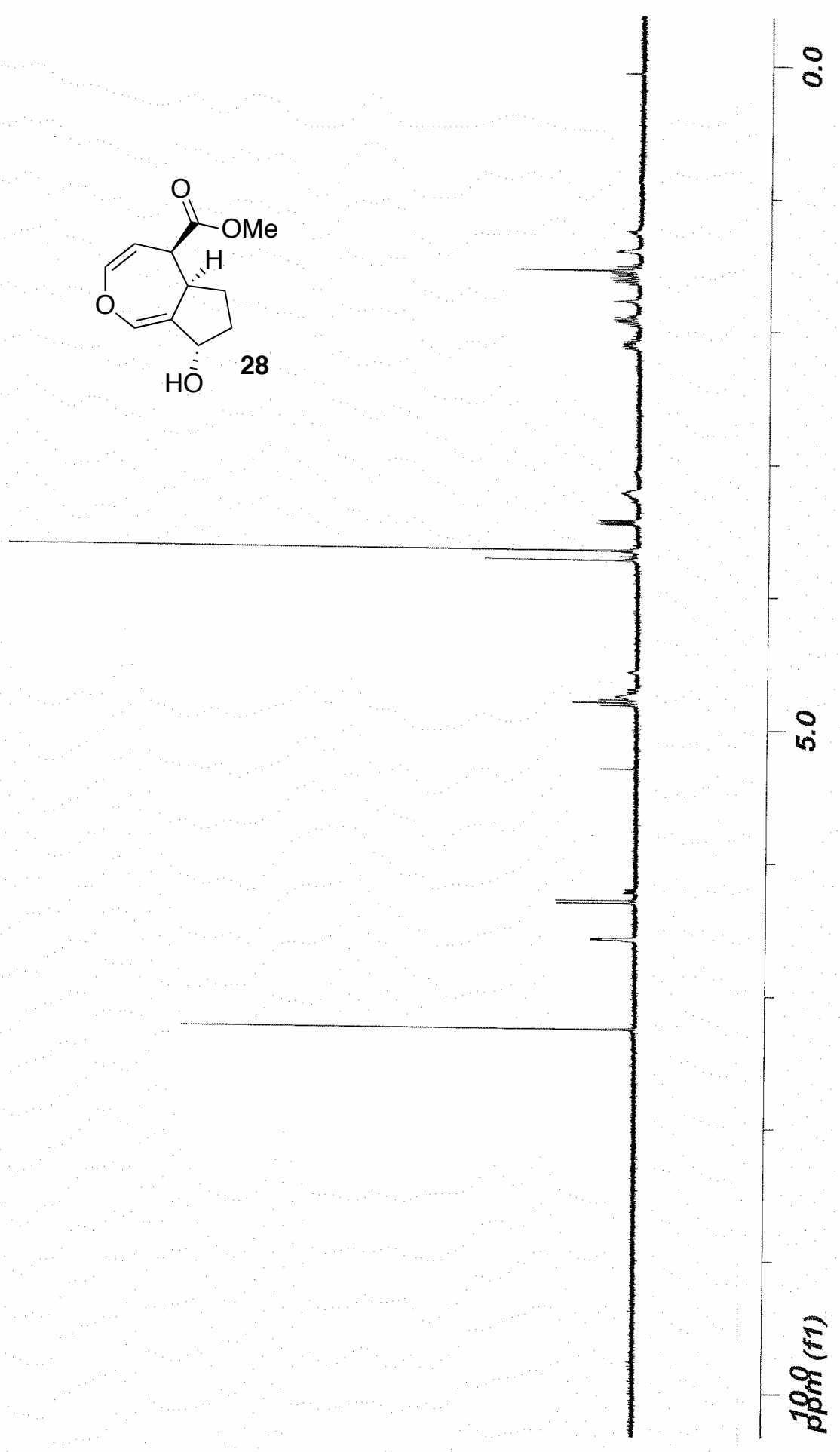




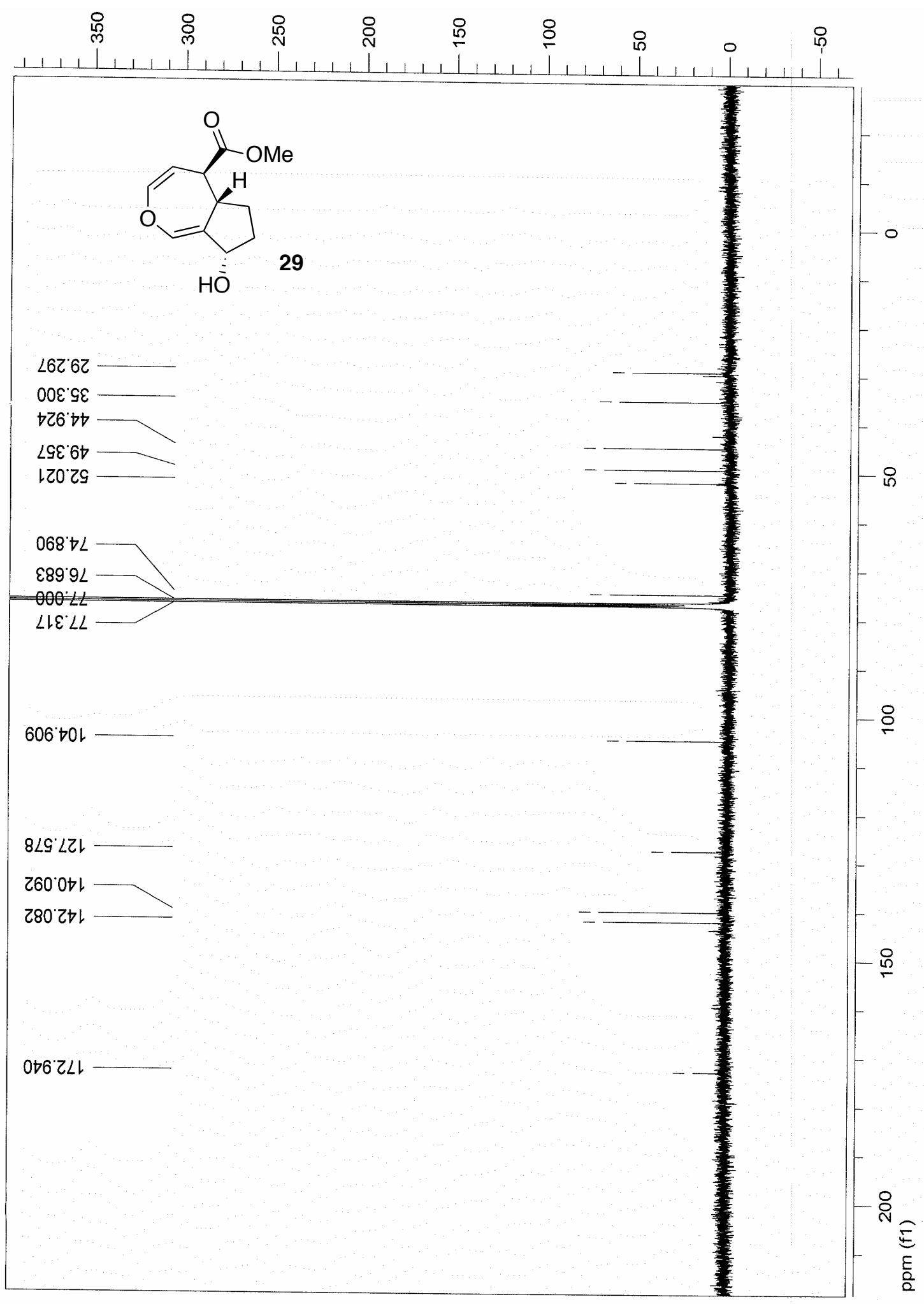




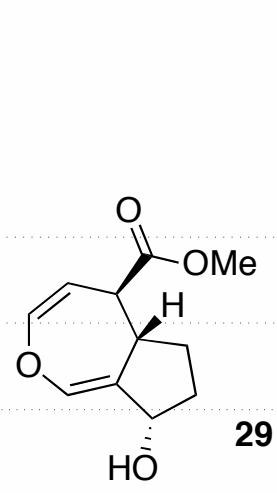

$\mid$\begin{tabular}{r|r} 
& \\
0 & 0
\end{tabular}

29

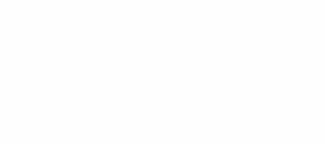

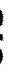
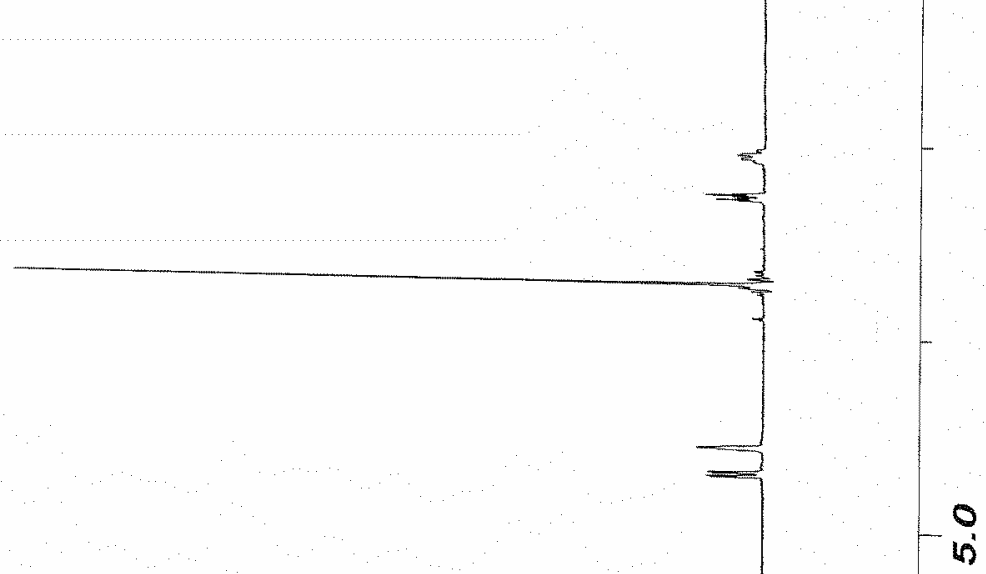

$\frac{\alpha}{\frac{\alpha}{2}}$ 


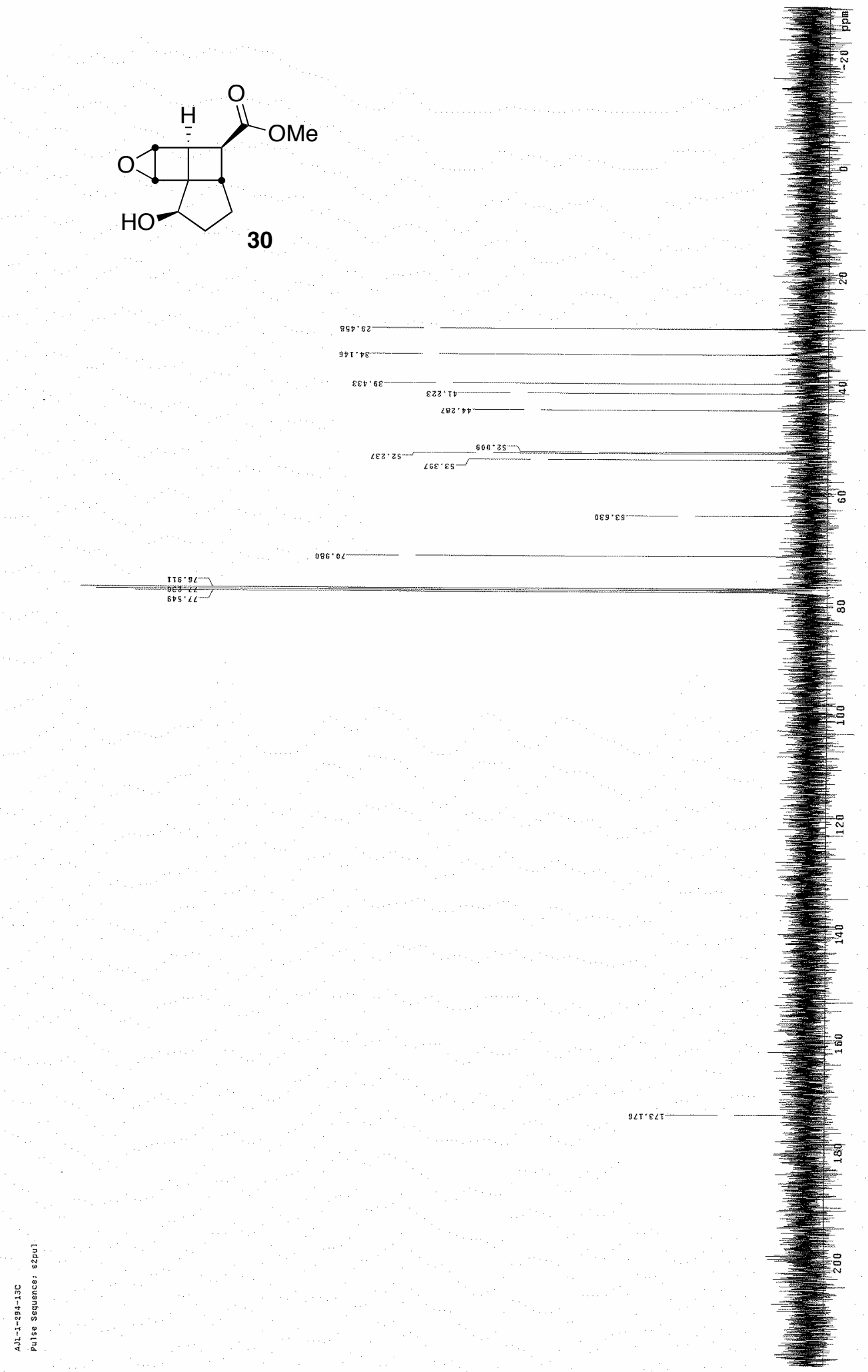




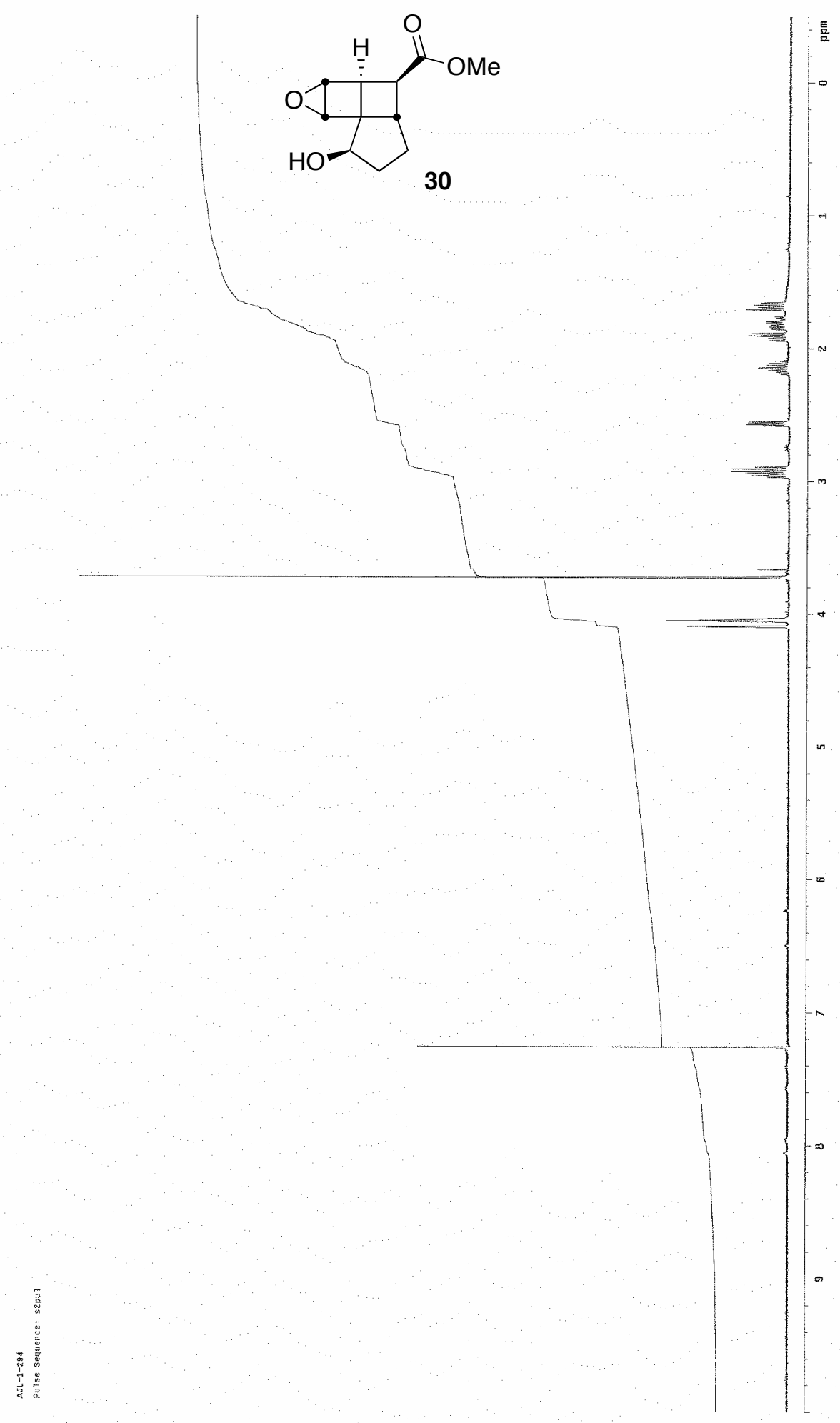



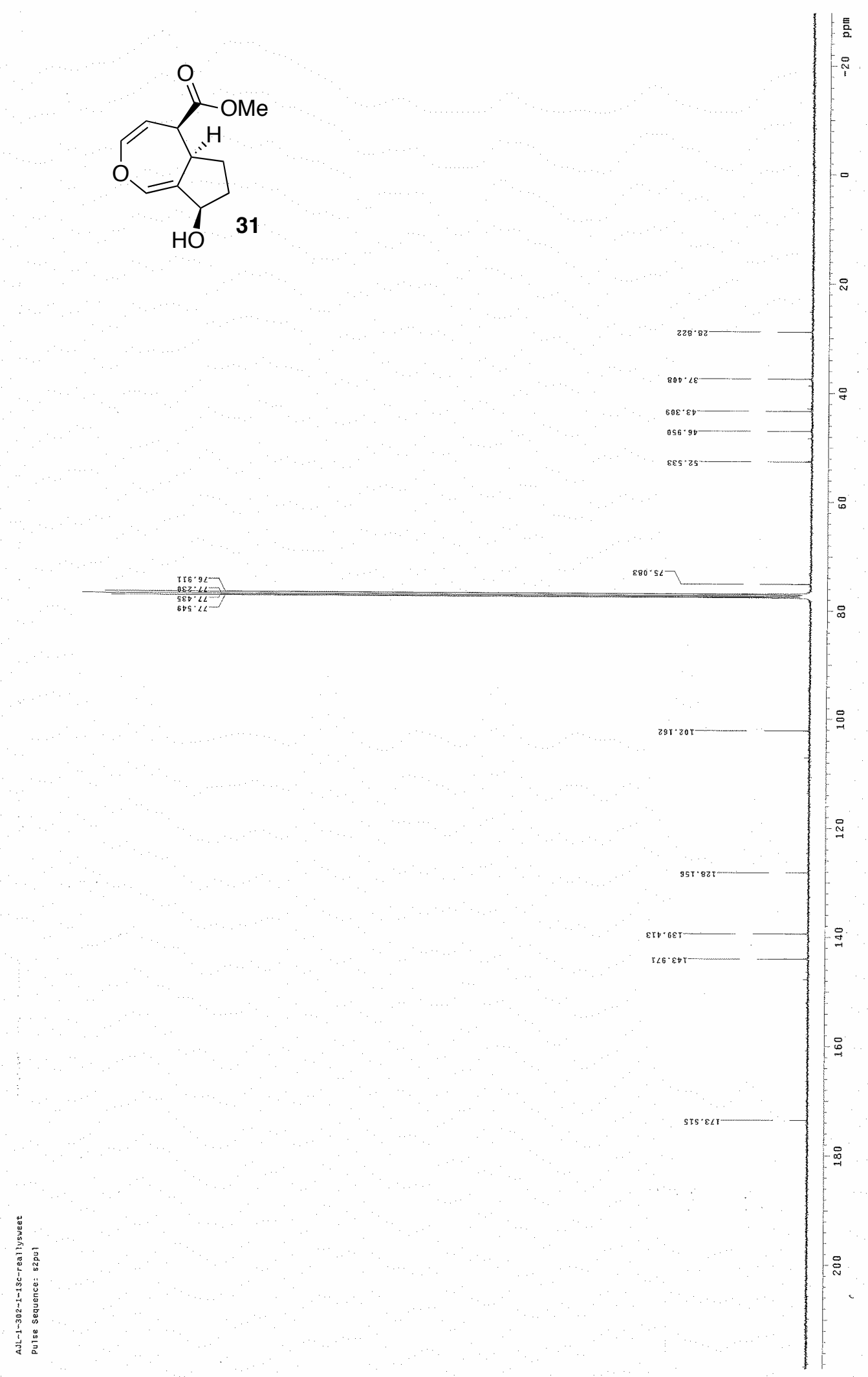


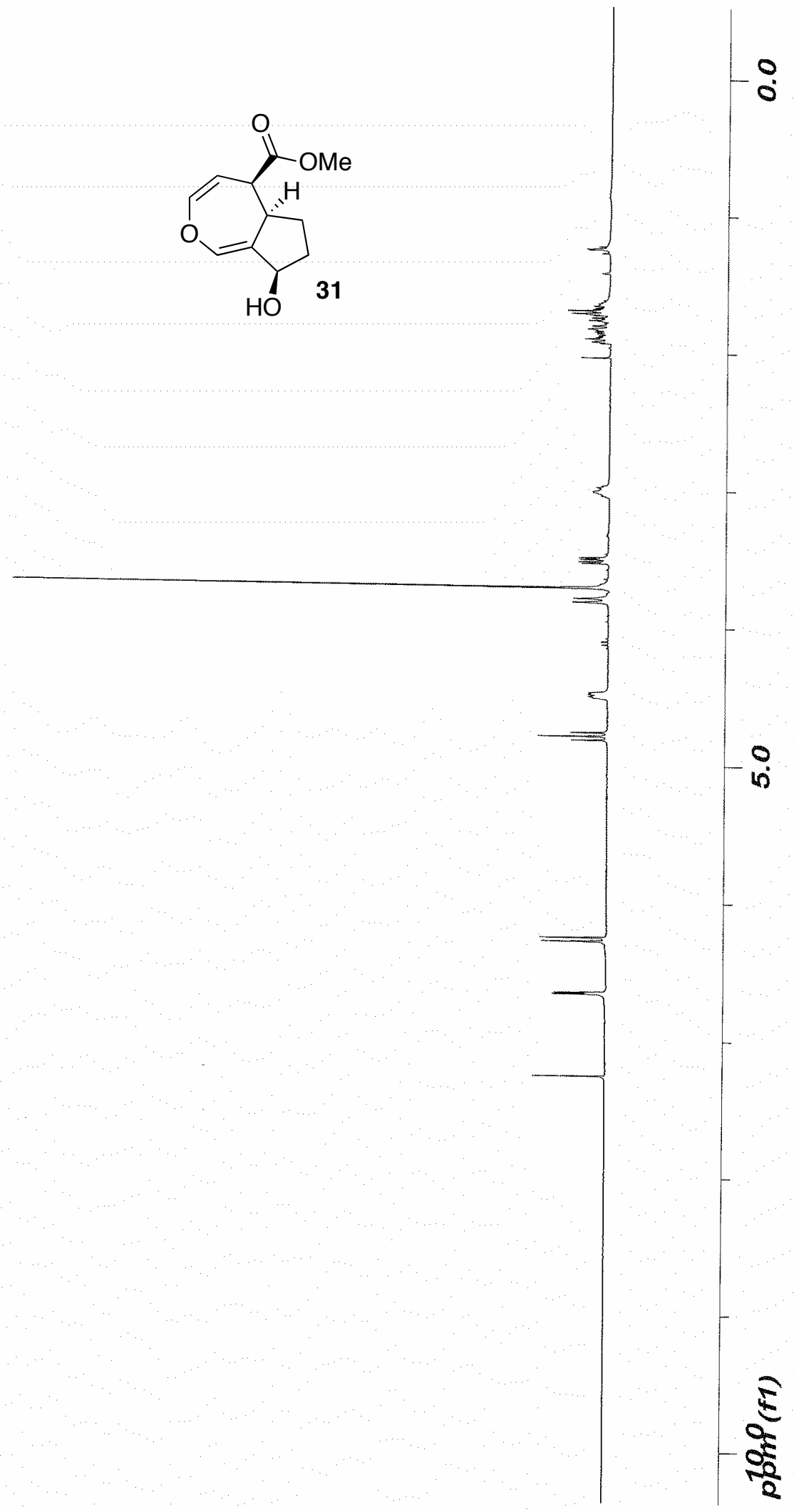




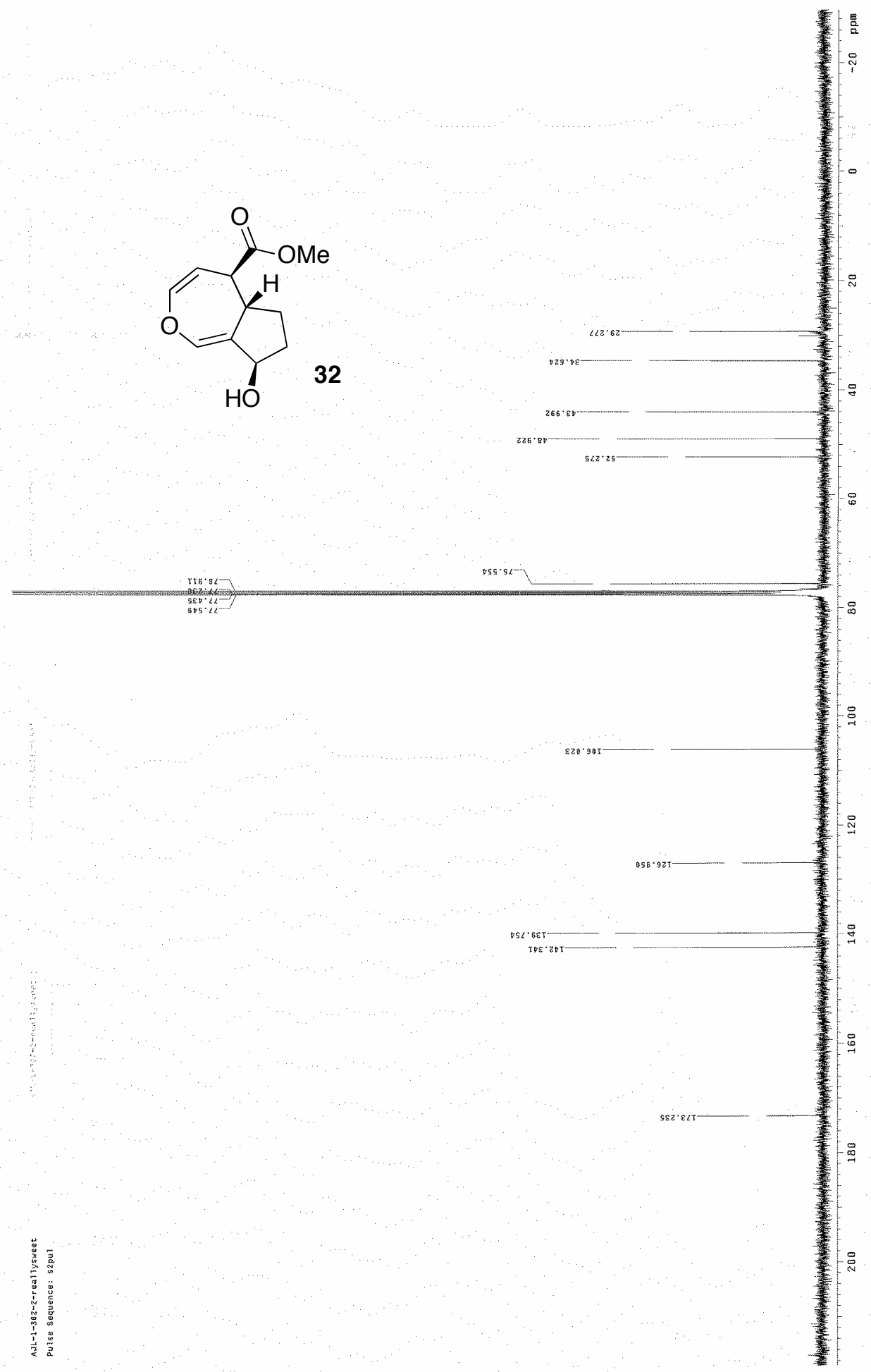



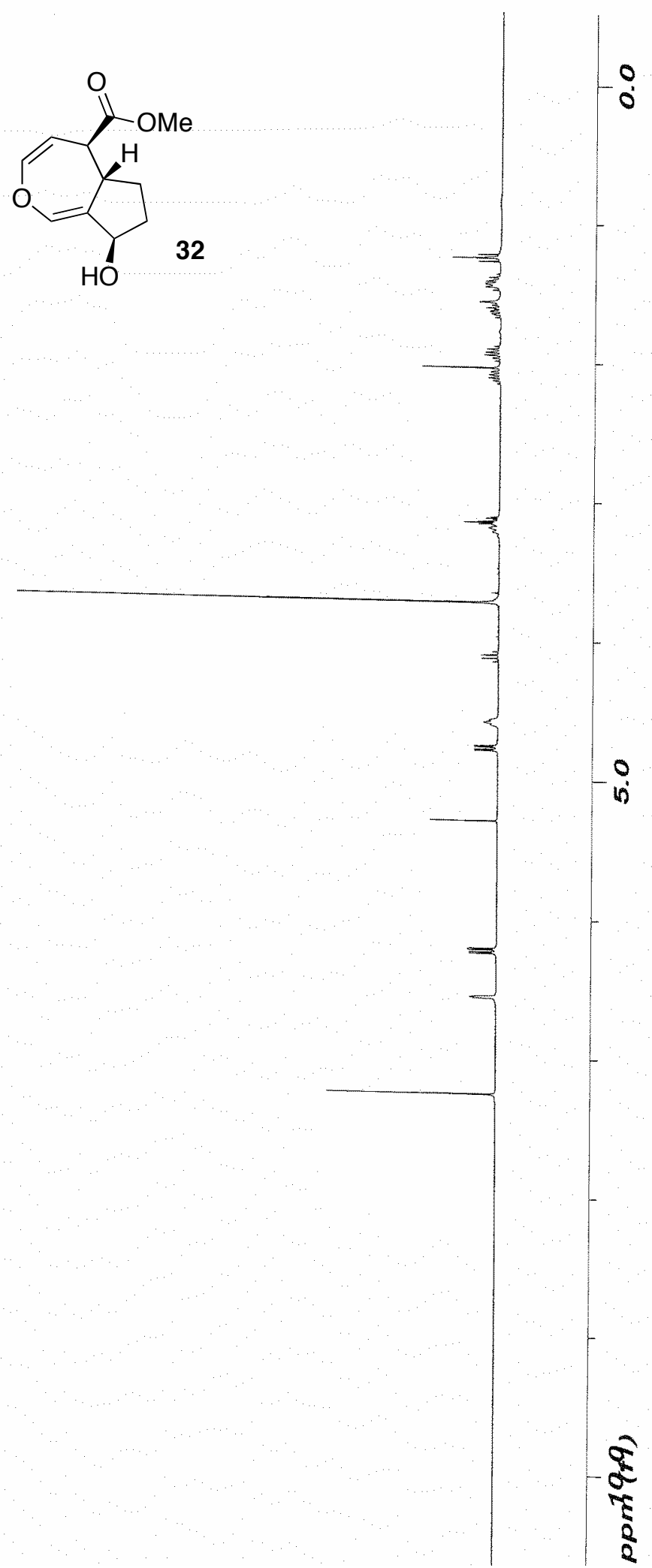

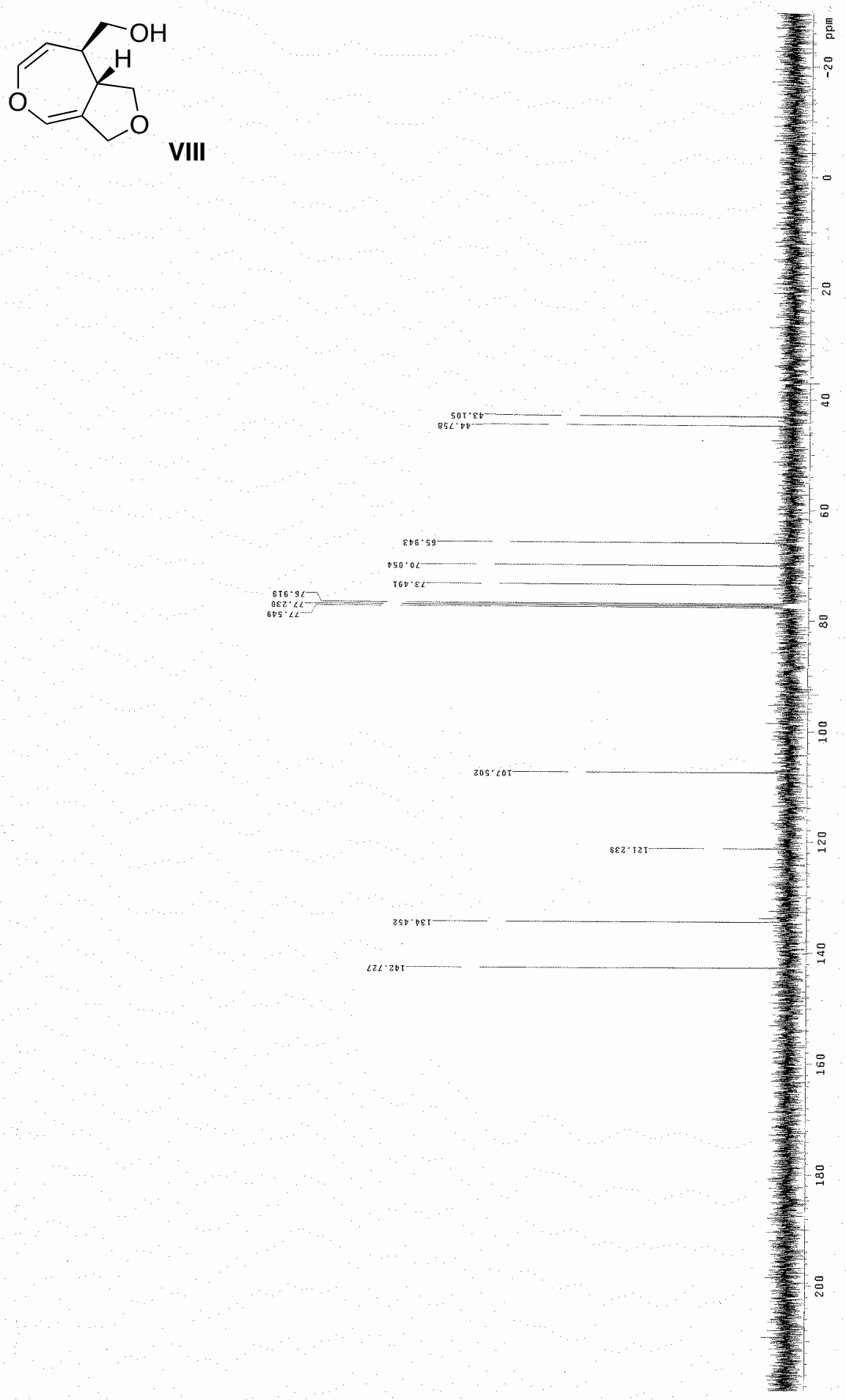


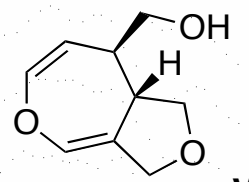

VIII

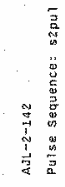
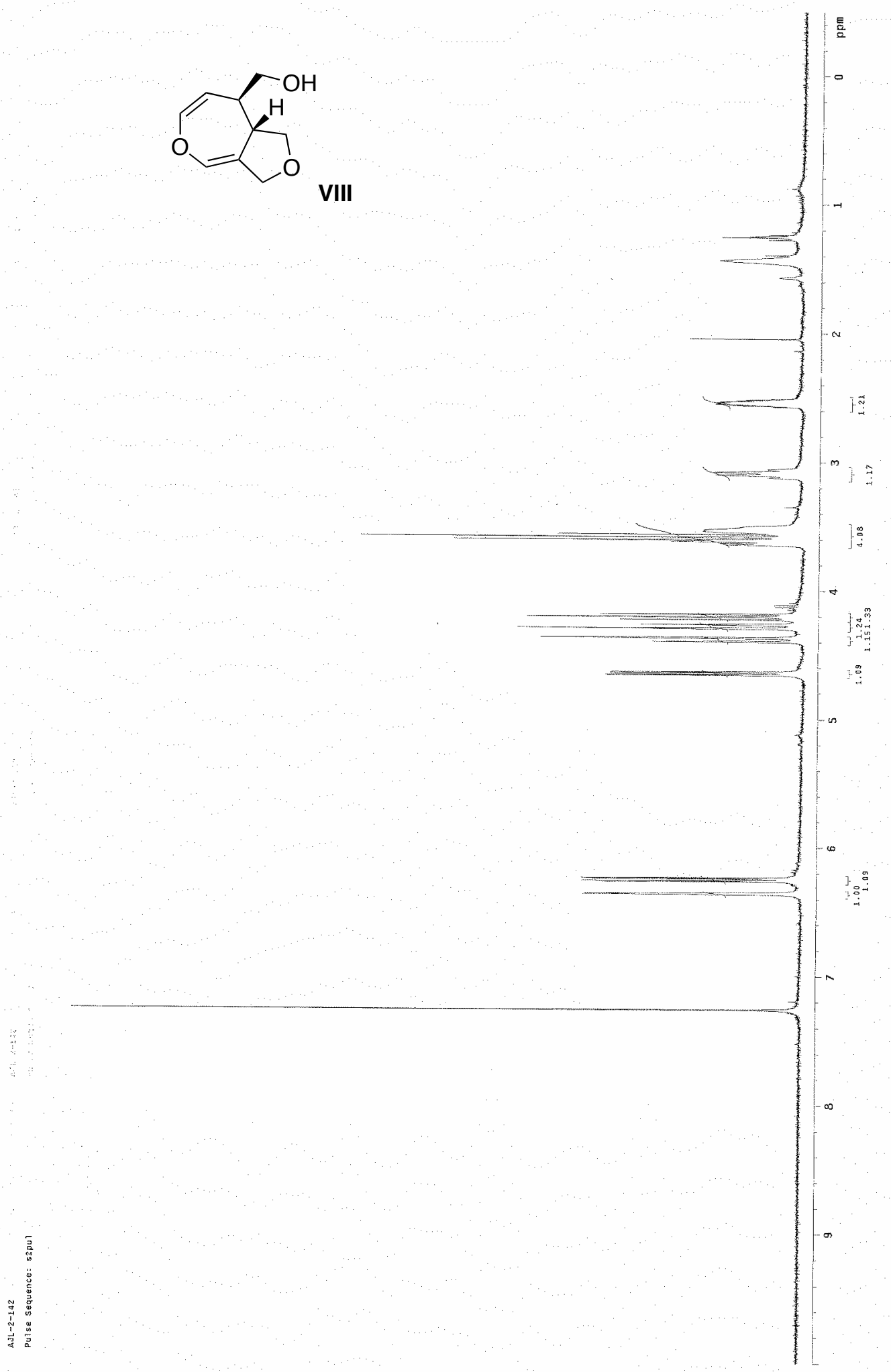

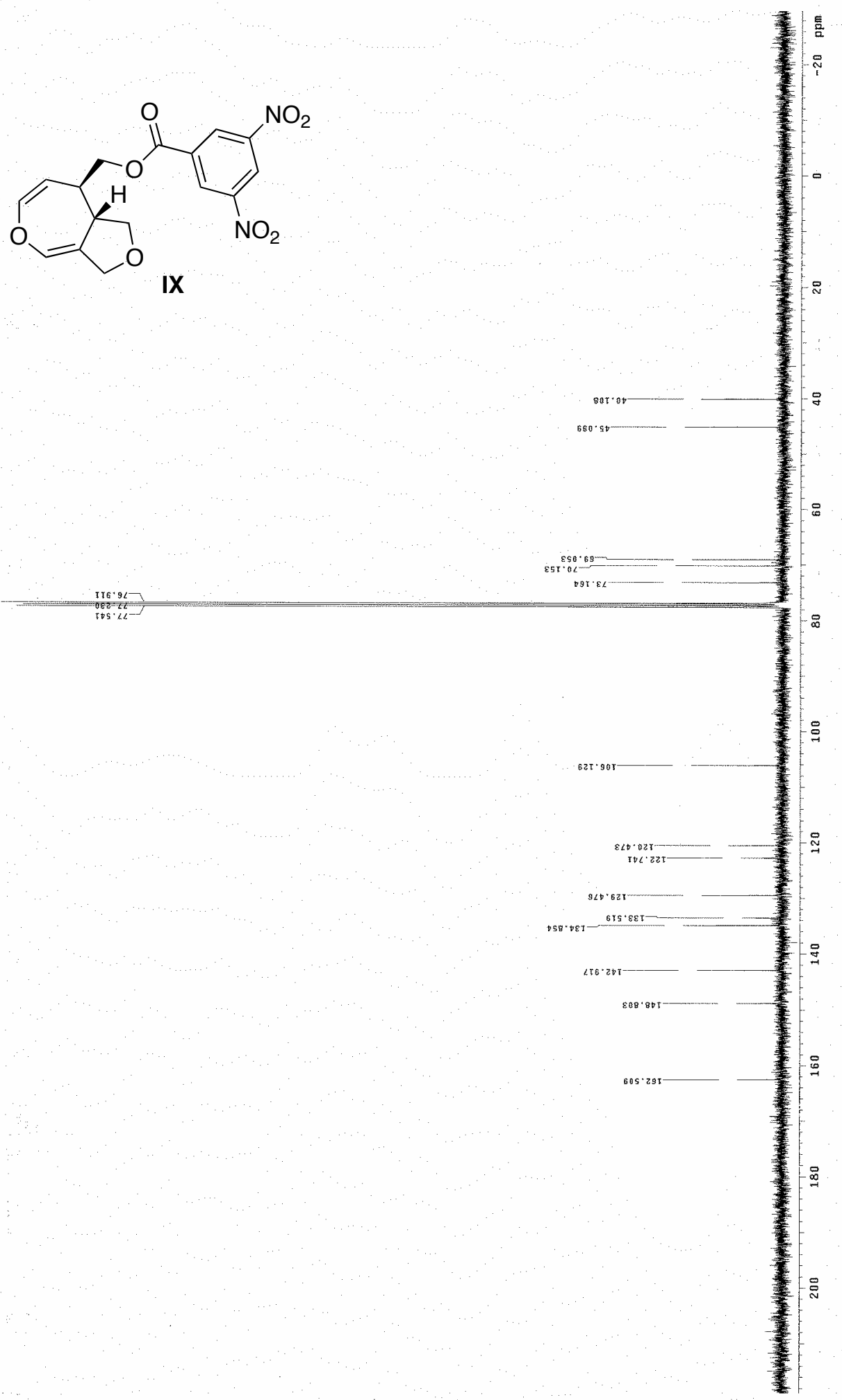

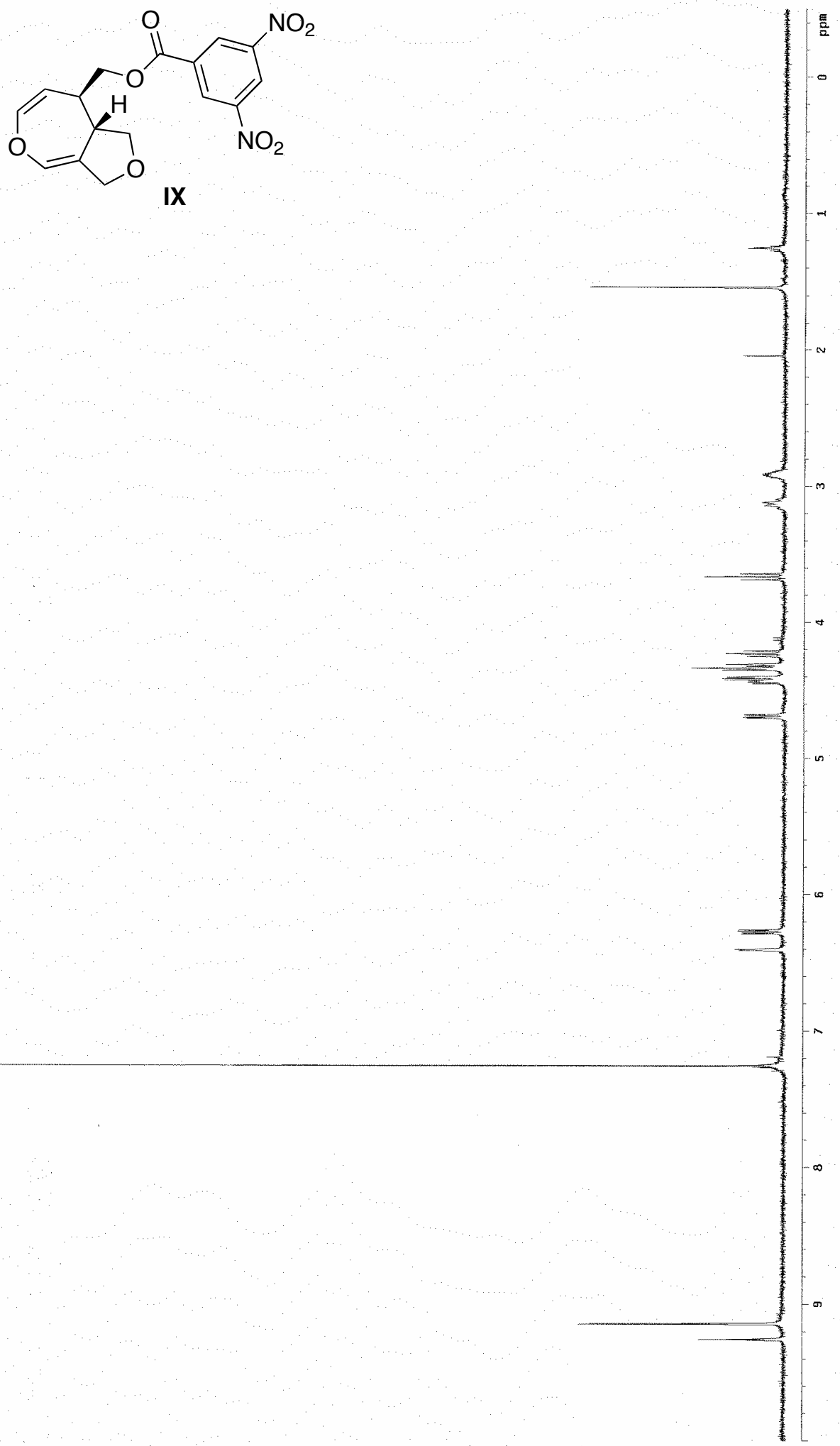


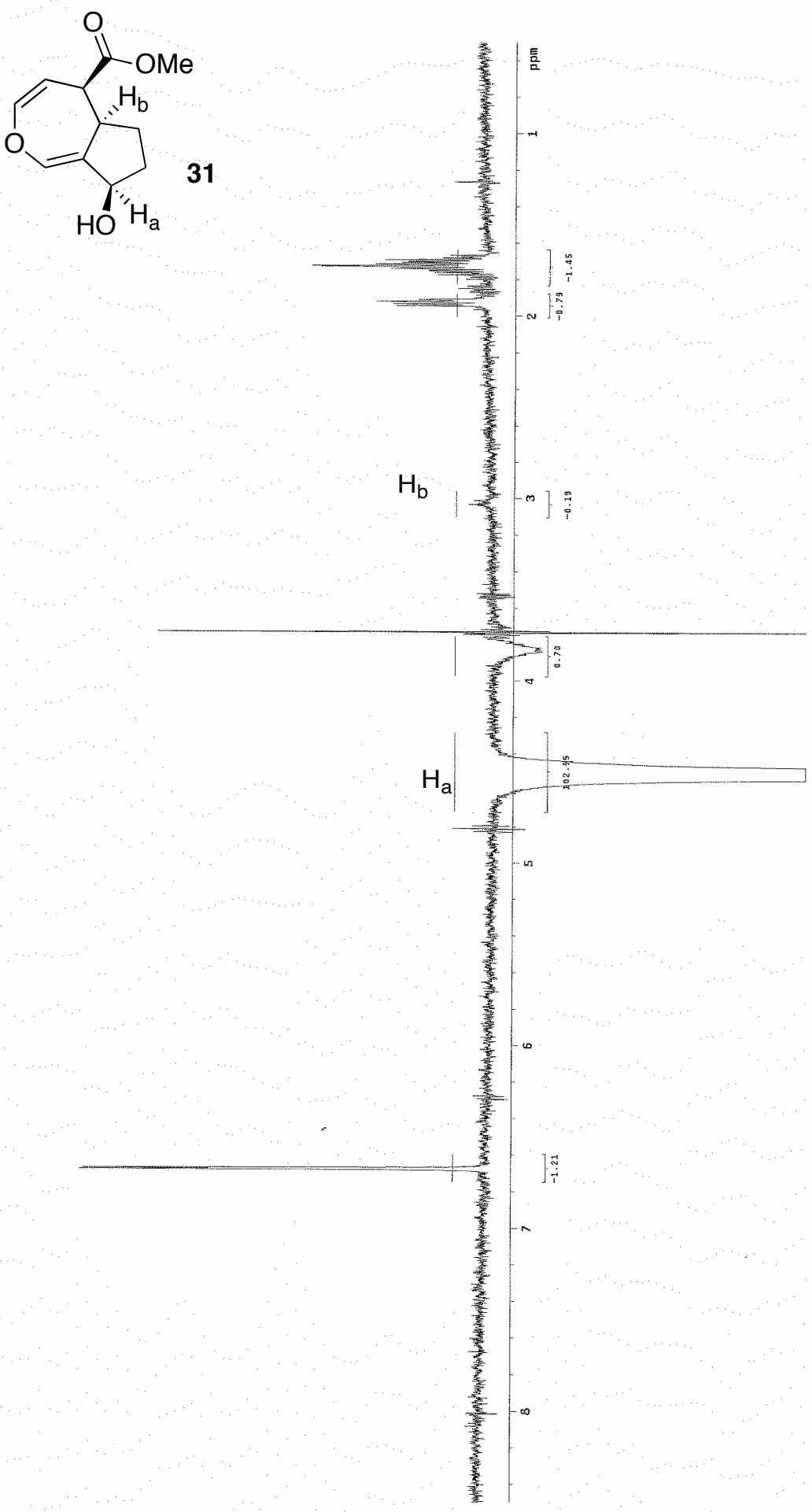




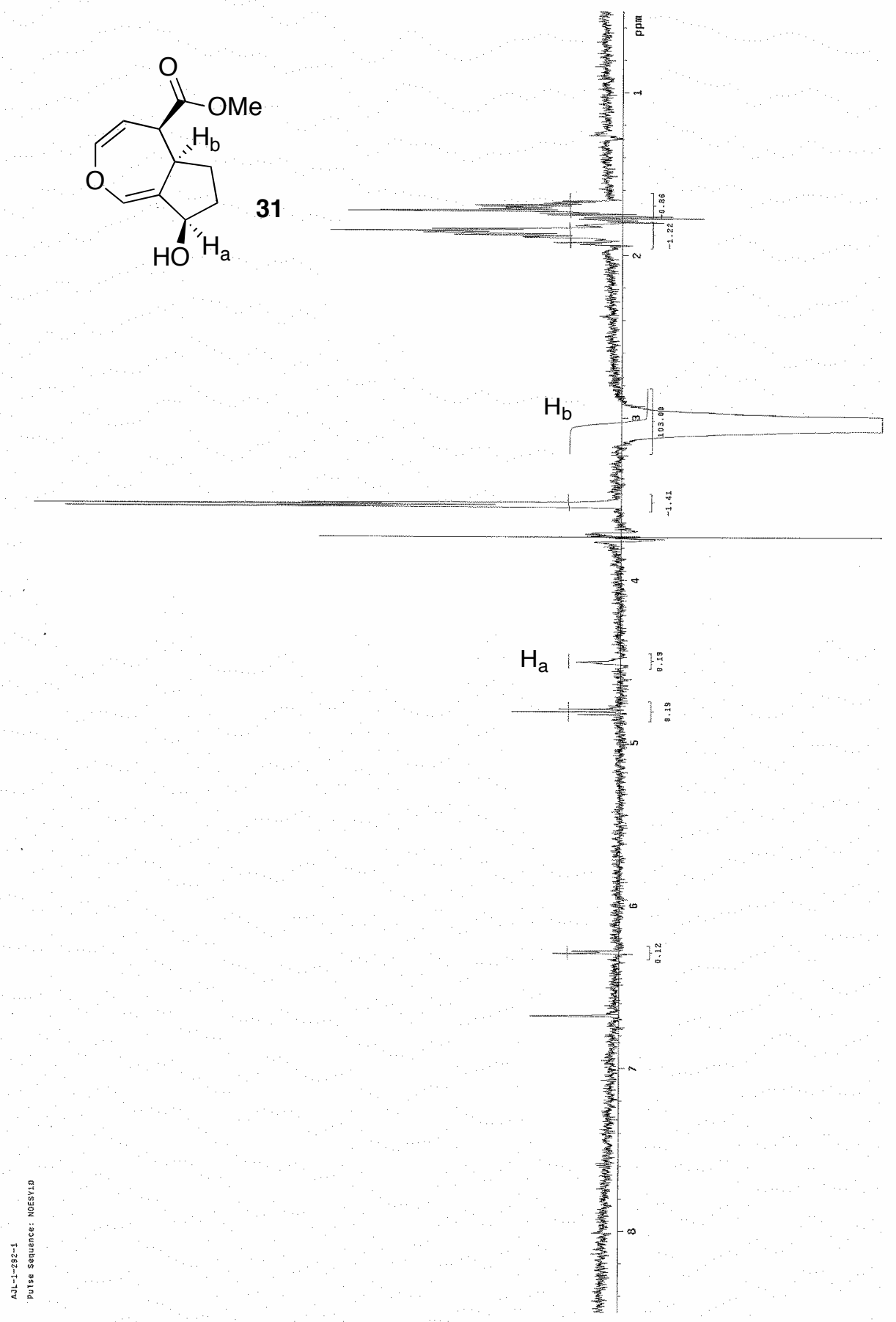




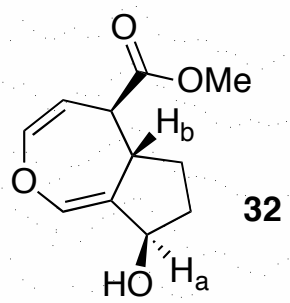

$\mathrm{H}_{\mathrm{a}}$
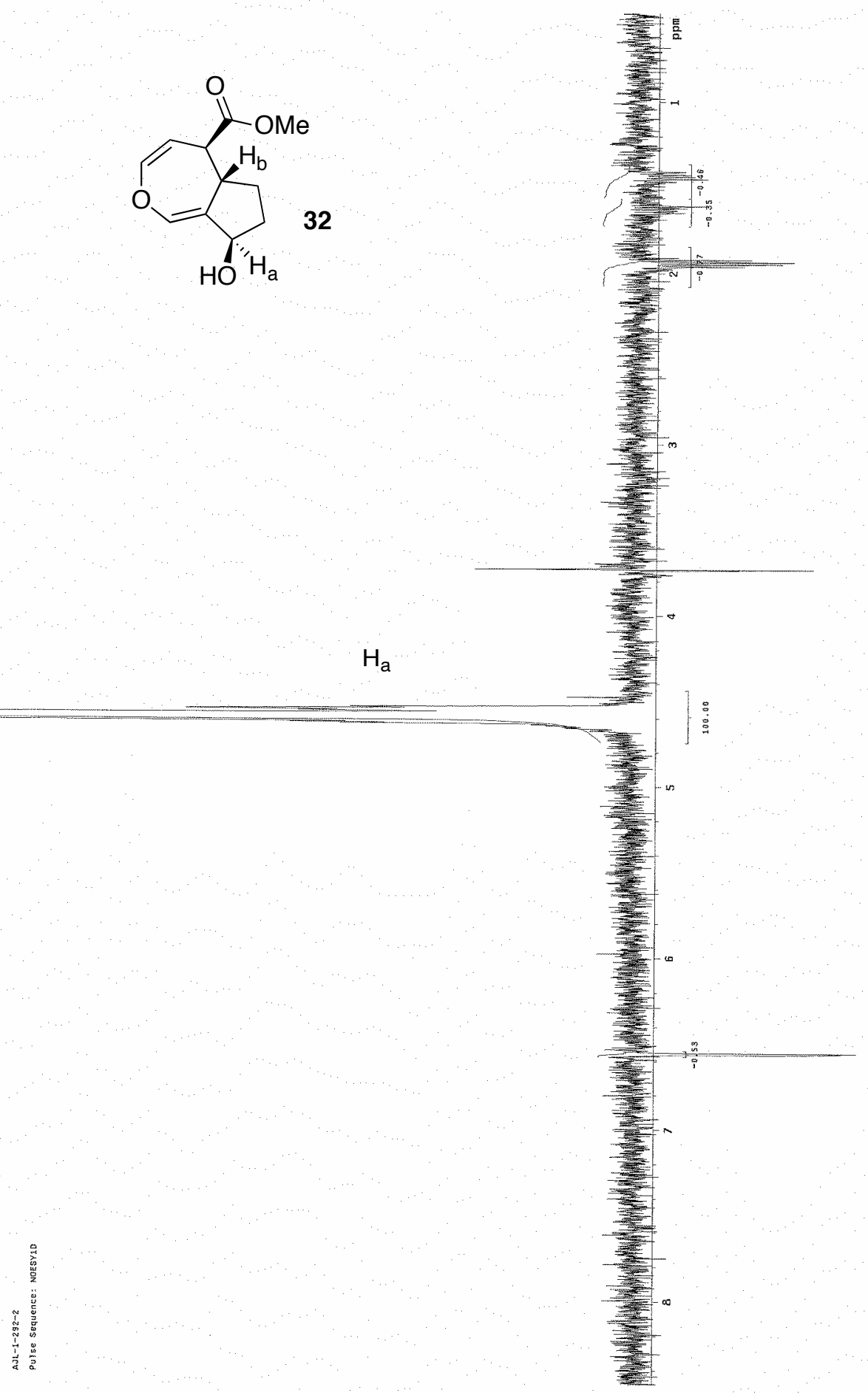


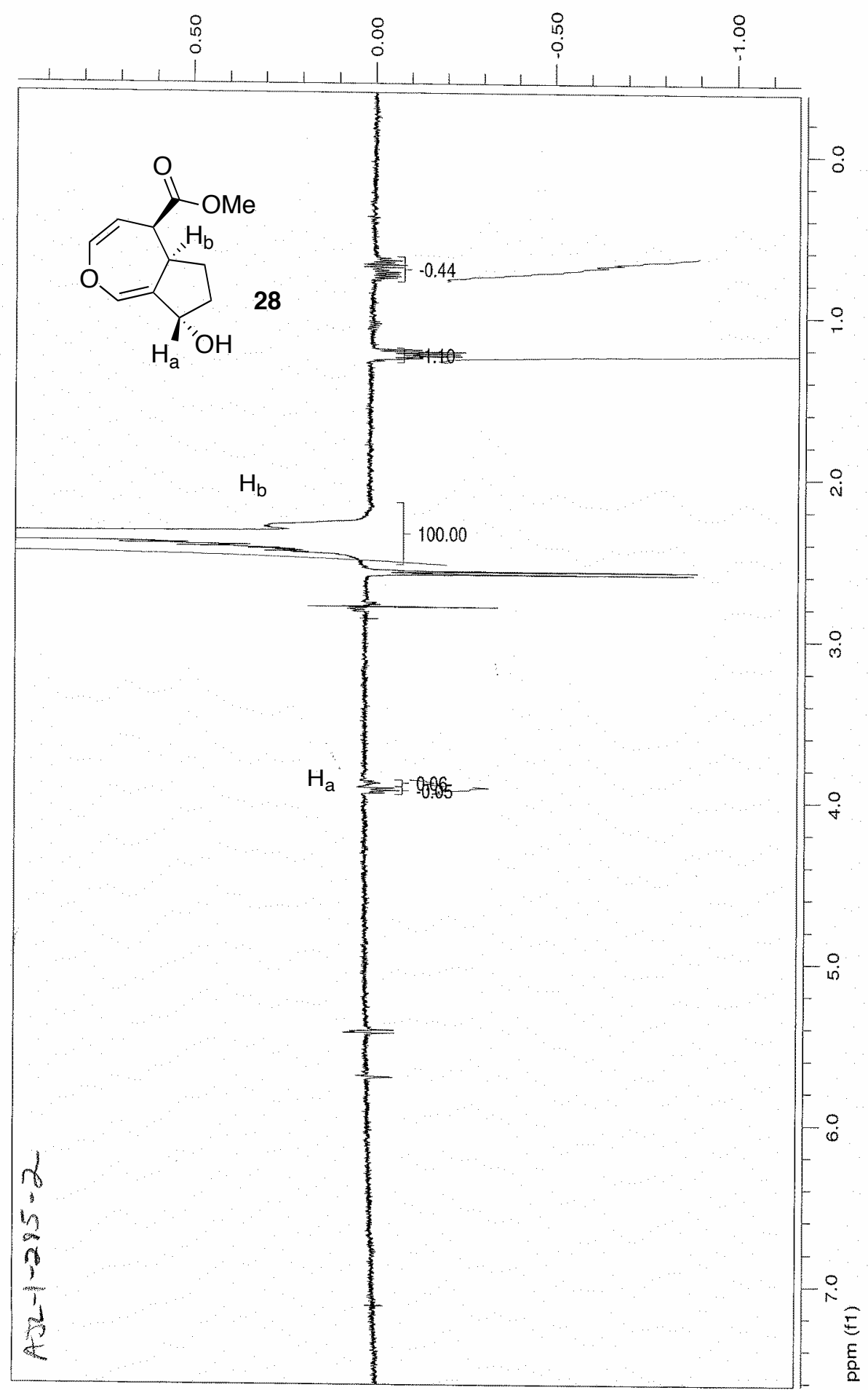




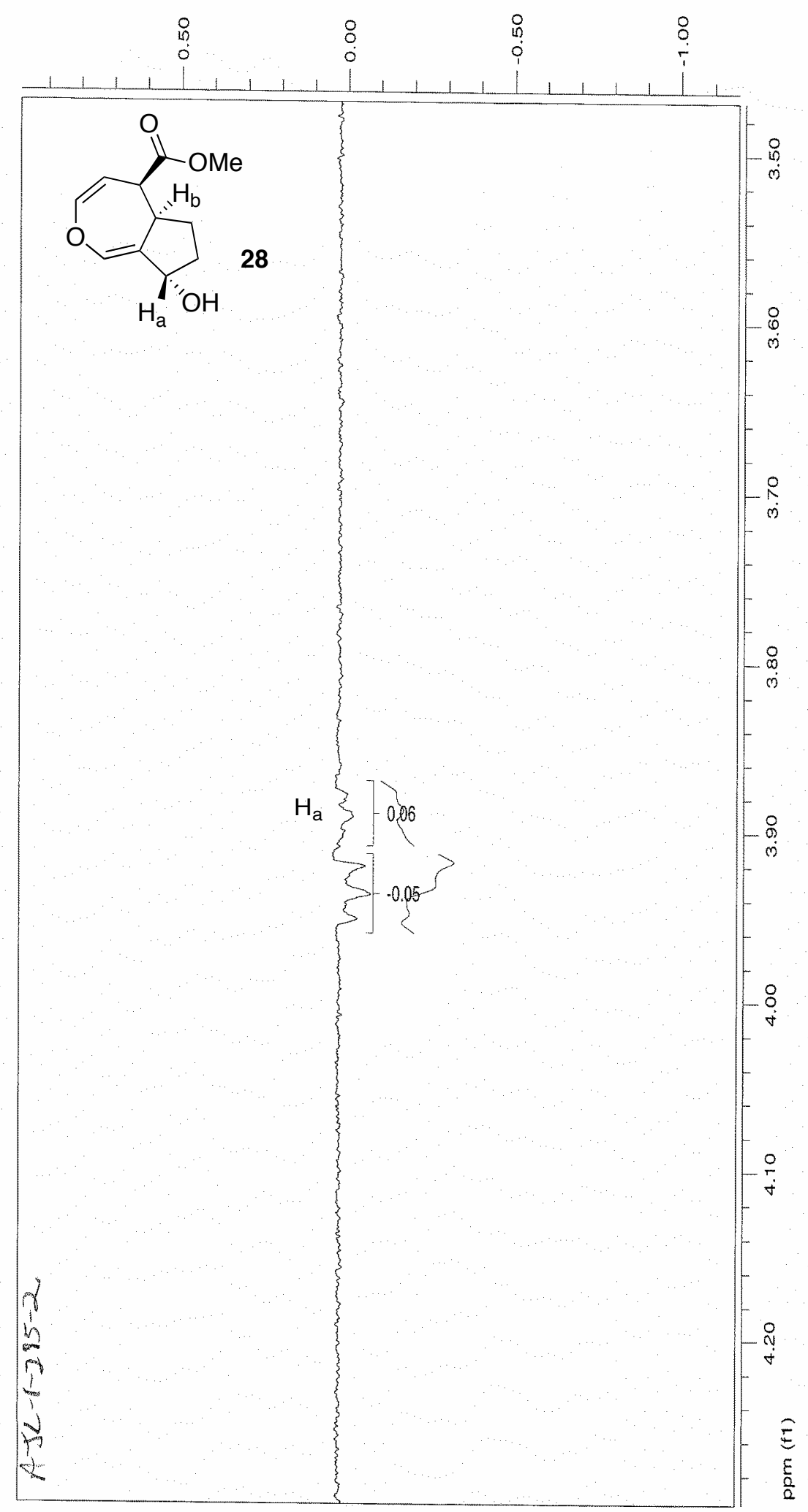




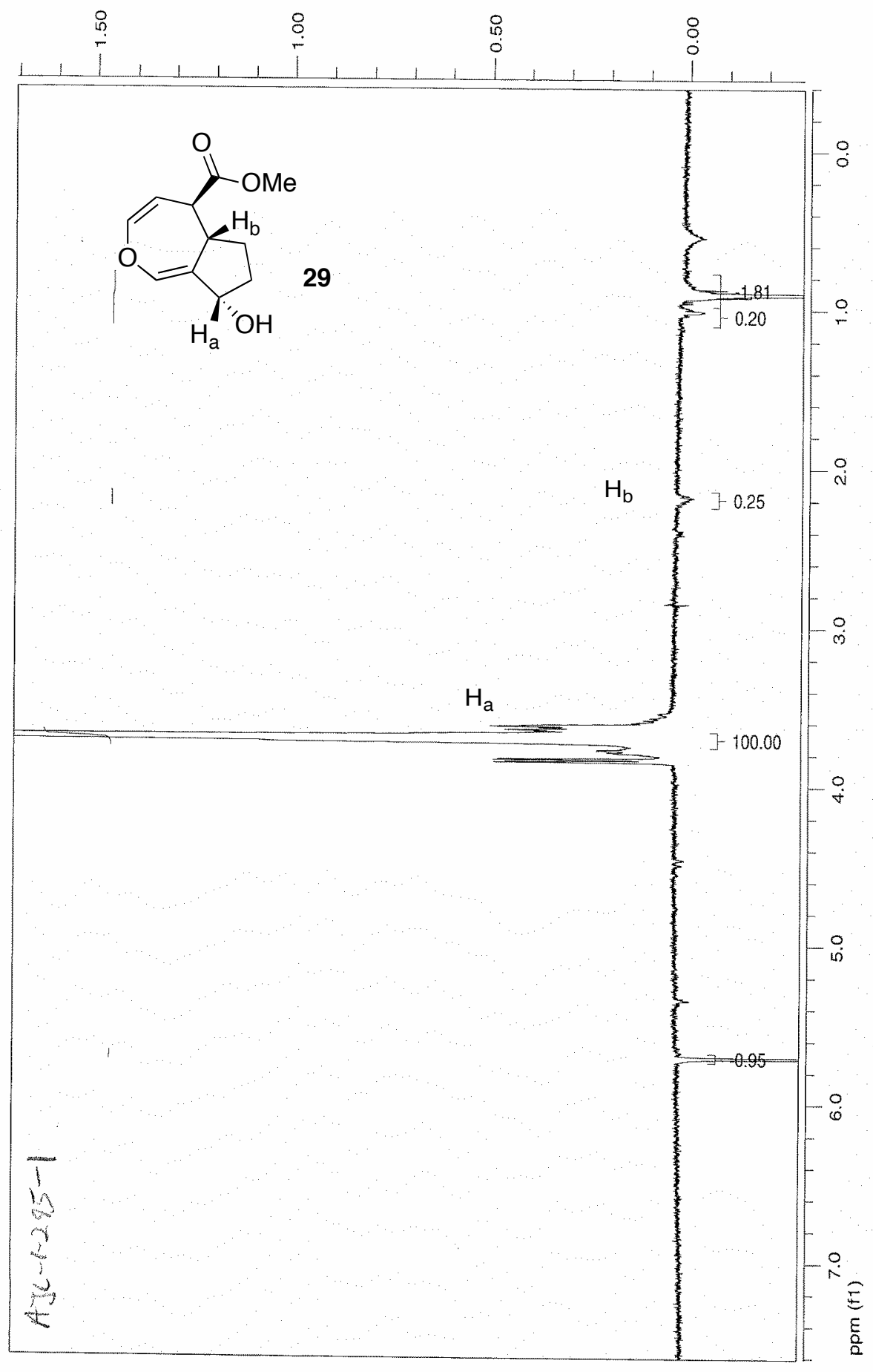

